Please do not remove this page

RMIT

UNIVERSITY

\title{
Airborne laser sensors and integrated systems
}

Sabatini, Roberto; Richardson, Mark; Gardi, Alessandro; Ramasamy, Subramanian

https://researchrepository.rmit.edu.au/esploro/outputs/9921862582201341/filesAndLinks?institution=61RMIT_INST\&index=null

Sabatini, R., Richardson, M., Gardi, A., \& Ramasamy, S. (2015). Airborne laser sensors and integrated systems. Progress in Aerospace Sciences, 79, 15-63. https://doi.org/10.1016/j.paerosci.2015.07.002 Document Version: Accepted Manuscript

Published Version: https://doi.org/10.1016/j.paerosci.2015.07.002

Repository homepage: https://researchrepository.rmit.edu.au (C) 2015 Elsevier Ltd.

Downloaded On 2023/04/26 19:26:44 +1000

Please do not remove this page 
Thank you for downloading this document from the RMIT Research Repository.

The RMIT Research Repository is an open access database showcasing the research outputs of RMIT University researchers.

RMIT Research Repository: http://researchbank.rmit.edu.au/

\section{Citation:}

Sabatini, R, Richardson, M, Gardi, A and Ramasamy, S 2015, 'Airborne laser sensors and integrated systems', Progress in Aerospace Sciences, pp. 1-48.

See this record in the RMIT Research Repository at:

https://researchbank.rmit.edu.au/view/rmit:33413

Version: Accepted Manuscript

Copyright Statement: (C) 2015 Elsevier Ltd.

Creative Commons Attribution-NonCommercial-NoDerivatives

4.0 International License

Link to Published Version:

http://dx.doi.org/10.1016/j.paerosci.2015.07.002 
This is the author pre-publication version. This paper does not include the changes arising from the revision, formatting and publishing process. The final paper that should be used for referencing is:

R. Sabatini, M. A. Richardson, A. Gardi, S. Ramasamy, "Airborne laser sensors and integrated systems", Progress in Aerospace Sciences (2015), http: //dx.doi.org/10.1016/j.paerosci.2015.07.002i.

\title{
Airborne laser sensors and integrated systems
}

\author{
Roberto Sabatini1*, Mark A. Richardson2, Alessandro Gardi1, Subramanian Ramasamy1 \\ RMIT University, Bundoora, Melbourne, Victoria 3083, Australia
}

Cranfield University, Defence Academy of the United Kingdom, Shrivenham, Swindon SN6 8LA, UK

\begin{abstract}
The underlying principles and technologies enabling the design and operation of airborne laser sensors are introduced and a detailed review of state-of-the-art avionic systems for civil and military applications is presented. Airborne lasers including Light Detection and Ranging (LIDAR), Laser Range Finders (LRF), and Laser Weapon Systems (LWS) are extensively used today and new promising technologies are being explored. Most laser systems are active devices that operate in a manner very similar to microwave radars but at much higher frequencies (e.g., LIDAR and LRF). Other devices (e.g., laser target designators and beam-riders) are used to precisely direct Laser Guided Weapons (LGW) against ground targets. The integration of both functions is often encountered in modern military avionics navigation-attack systems. The beneficial effects of airborne lasers including the use of smaller components and remarkable angular resolution have resulted in a host of manned and unmanned aircraft applications. On the other hand, laser sensors performance are much more sensitive to the vagaries of the atmosphere and are thus generally restricted to shorter ranges than microwave systems. Hence it is of paramount importance to analyse the performance of laser sensors and systems in various weather and environmental conditions. Additionally, it is important to define airborne laser safety criteria, since several systems currently in service operate in the near infrared with considerable risk for the naked human eye. Therefore, appropriate methods for predicting and evaluating the performance of infrared laser sensors/systems are presented, taking into account laser safety issues. For aircraft experimental activities with laser systems, it is essential to define test requirements taking into account the specific conditions for operational employment of the systems in the intended scenarios and to verify the performance in realistic environments at the test ranges. To support the development of such requirements, useful guide lines are provided for test and evaluation of airborne laser systems including laboratory, ground and flight test activities.
\end{abstract}

\section{Keywords:}

Airborne lasers, Laser sensors, Laser systems, Avionics, Electro-optics, Aerospace electronic systems, Photonics, Laser performance analysis, Laser eye-safety, Laser test and evaluation

\section{Contents}

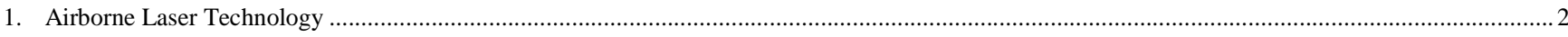

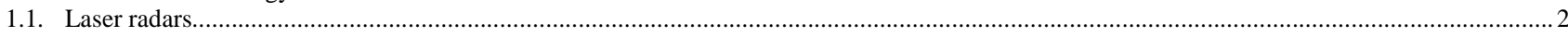

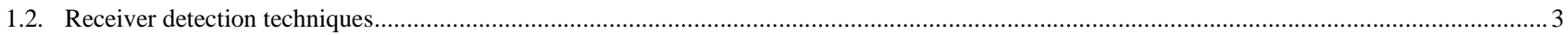

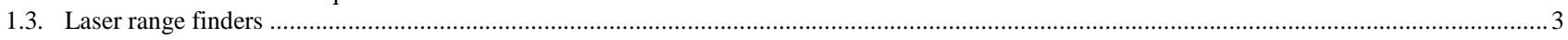

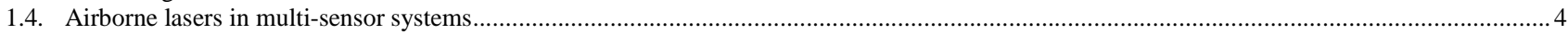

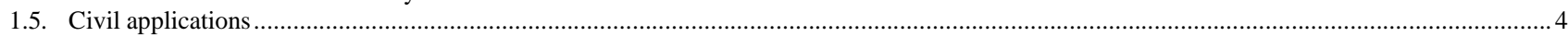

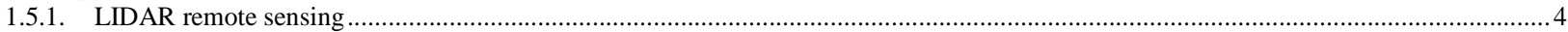

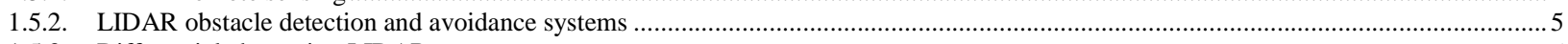

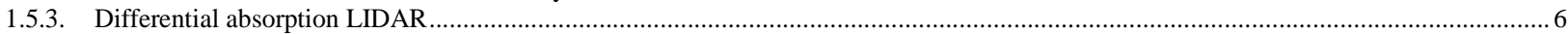

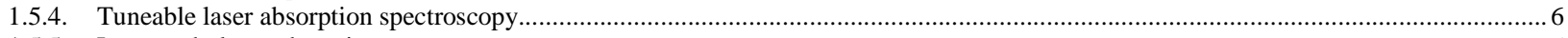

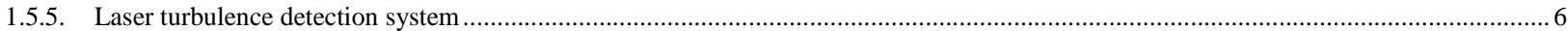

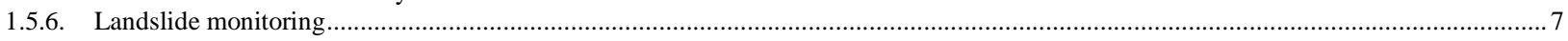

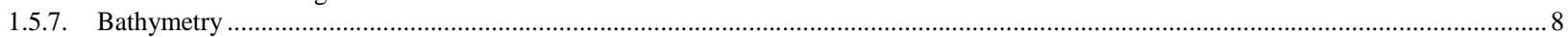

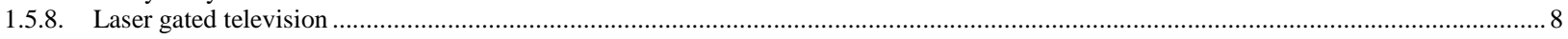

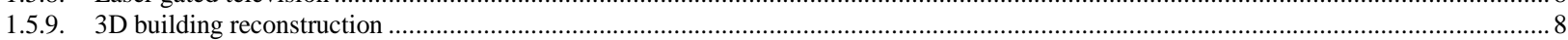

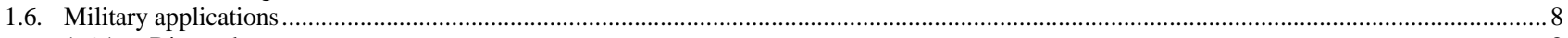

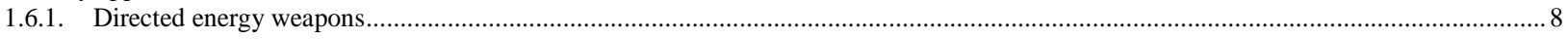

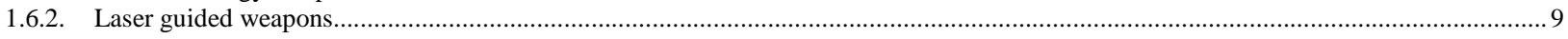

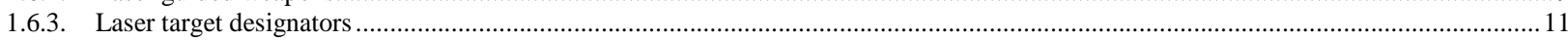

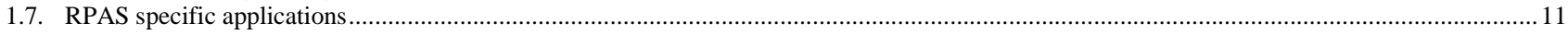

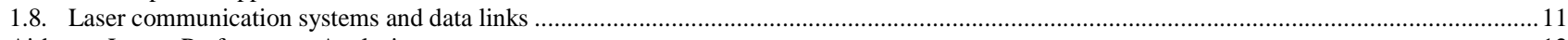

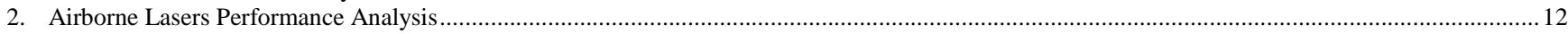

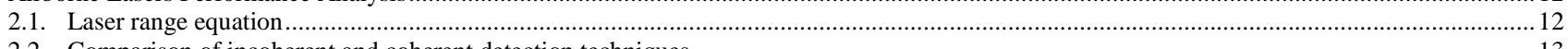

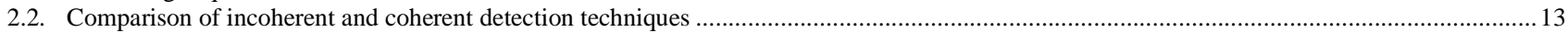

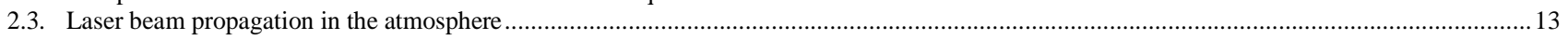

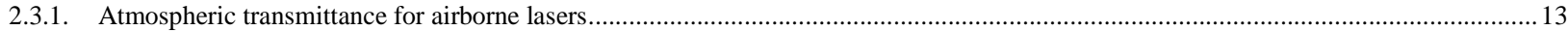

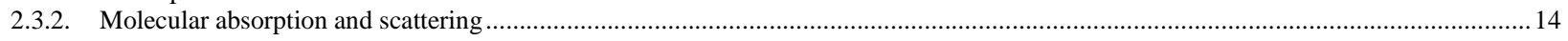

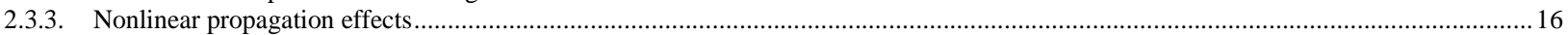

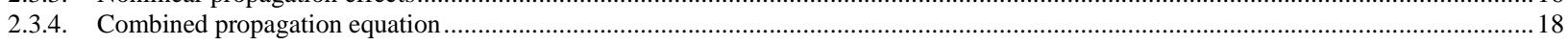

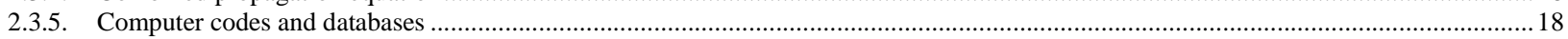

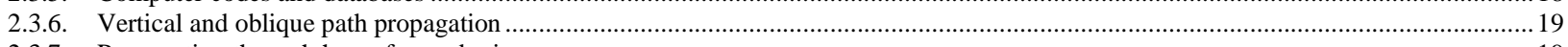

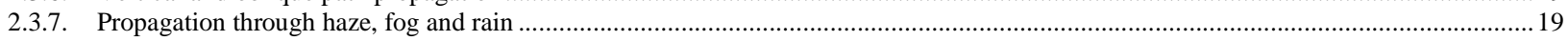

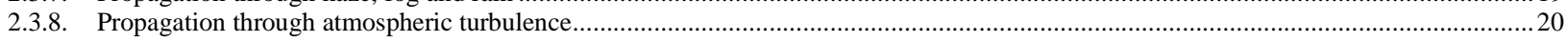

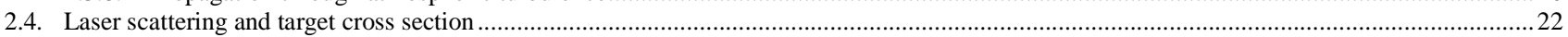

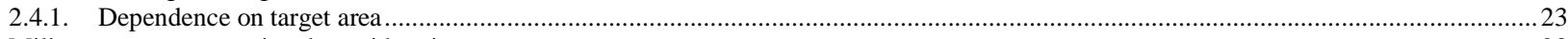

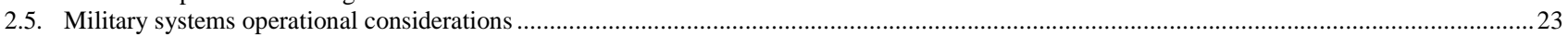

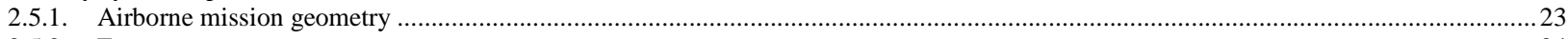

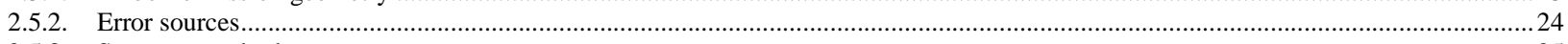

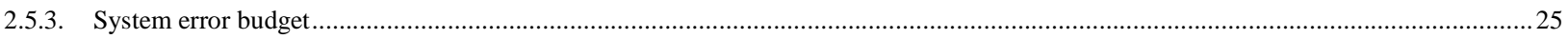

\footnotetext{
* Corresponding author.

E-mail address: roberto.sabatini@rmit.edu.au (R. Sabatini)
} 
This is the author pre-publication version. This paper does not include the changes arising from the revision, formatting and publishing process. The final paper that should be used for referencing is:

R. Sabatini, M. A. Richardson, A. Gardi, S. Ramasamy, "Airborne laser sensors and integrated systems", Progress in Aerospace Sciences (2015), http: //dx.doi.org/10.1016/j.paerosci.2015.07.002i.

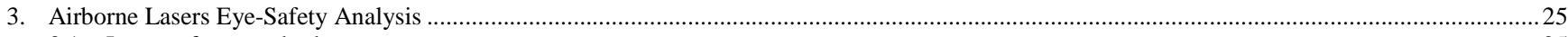

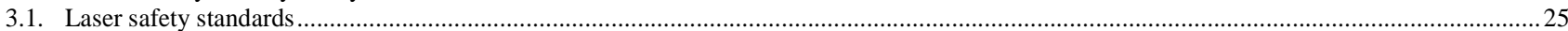

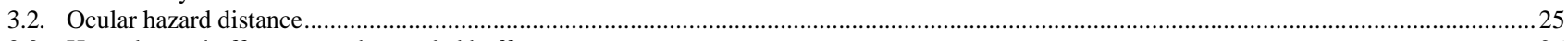

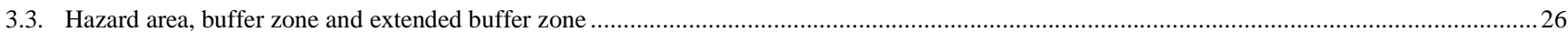

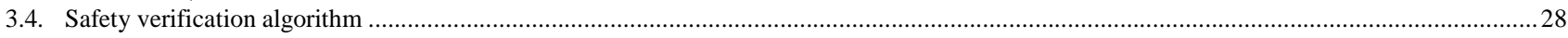

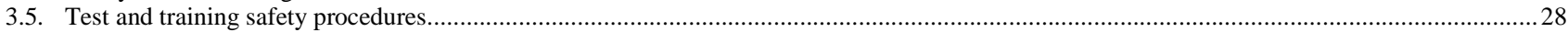

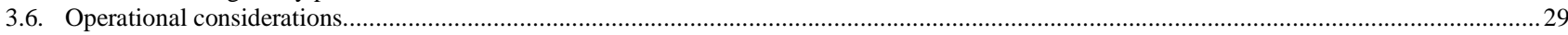

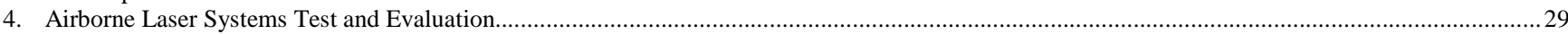

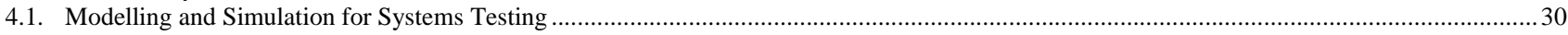

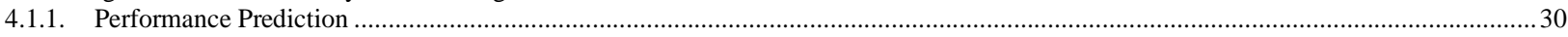

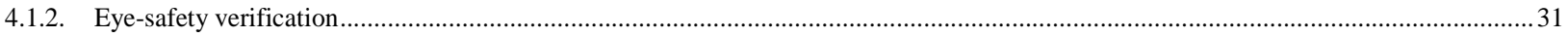

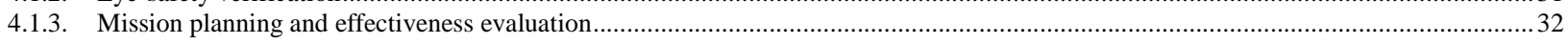

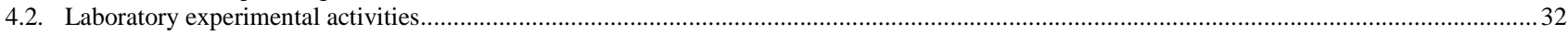

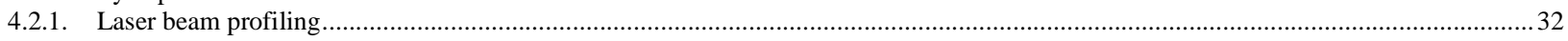

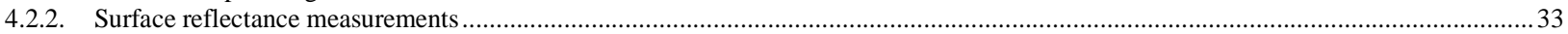

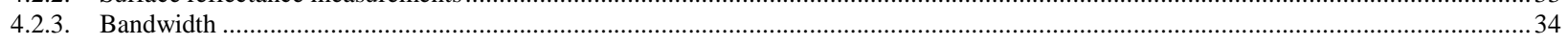

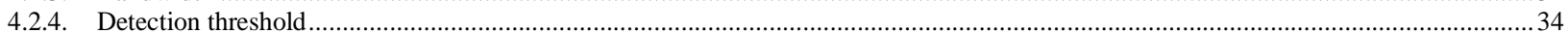

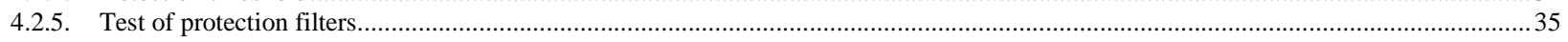

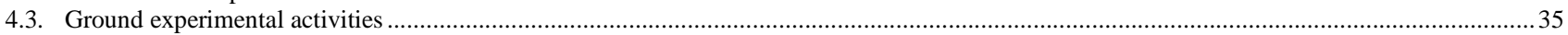

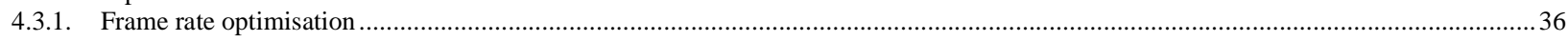

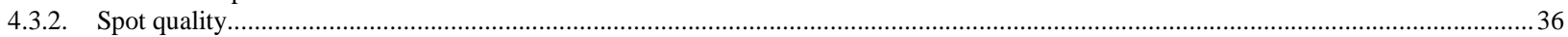

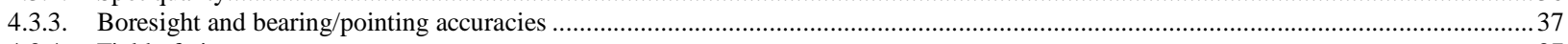

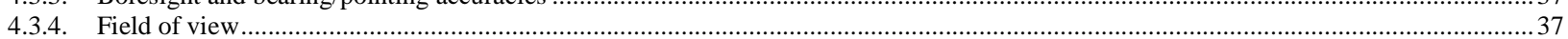

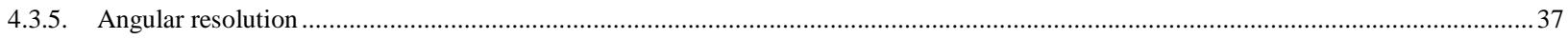

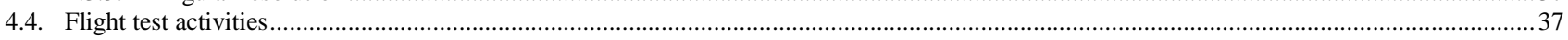

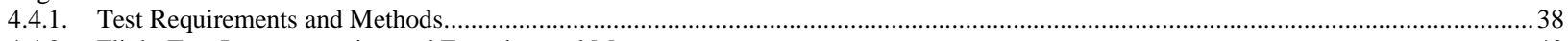

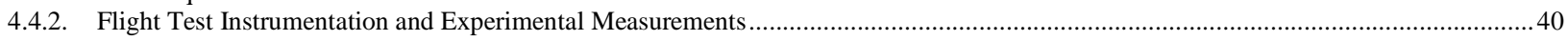

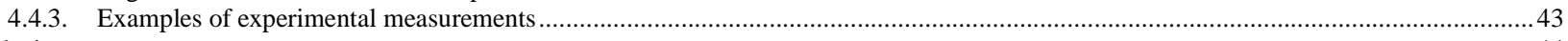

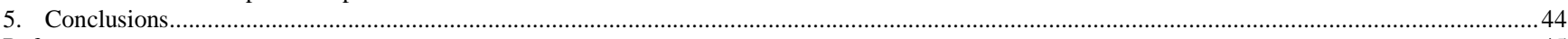

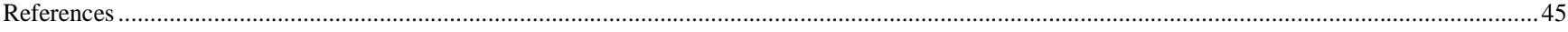

\section{Airborne Laser Technology}

Throughout the 1950s, significant research efforts were devoted to laser technology and some practical applications started to emerge. Since then, a large number of R\&D programmes have been carried out on lasers, which have led to a rich diversity of systems, ranging from laboratory devices used for studying nonlinear optical emissions and propagation, to eye-safe, compact and inexpensive laser-ranging binoculars. Over the years, military interests in airborne laser systems have concentrated in four general areas: Laser Rangefinders (LRF) and Target Designators (LTD), laser radars (Light Detection and Ranging - LIDAR), Laser Communication Systems (LCS) and Directed Energy Weapons (DEW). Although military lasers are significantly different from those in the public domain, the transfer of military technologies (i.e. powerful laser sources and other electro-optical devices) has resulted innumerous civil applications, including 3D mapping, turbulence detection, wireless power transmission and ground obstacle avoidance, to mention a few [1-3].

\subsection{Laser radars}

The development of LIDAR systems commenced in the 1960s with early systems built for ranging applications [4]. In the 1980s, a variety of remote sensing applications flourished and LIDAR technology started to be adopted in airborne installations to map the terrain elevation [5]. In 1988, the U.S. Army Corps of Engineers (USACE) assembled an operational LIDAR system [6], while the Australian Laser Airborne Depth Sounder (LADS) system was developed [7]. With advancements in electronics and the introduction of Global Navigation Satellite System (GNSS) for georeferencing, the number of airborne LIDAR applications has seen a steady increase. LIDAR sensors can be classified in three main categories, namely discrete return, full waveform and profiling. The simplest of the three is profiling, wherein the record of only one return is performed. The more advanced discrete return type sensors record multiple returns while the waveform sensors record a digitized profile of the full return pulse. An improved positional accuracy is obtained from the LIDAR systems only when reliable and precise information of the aircraft location is known at both the transmission and reception times. State-of-the-art LIDAR systems are capable of capturing the reflected signal, as well as providing georeferencing of the three-dimensional coordinates of the laser returns [8-10]. The basic concept of operation of a LIDAR is identical to that of conventional radar. The laser source emits a signal that is reflected by a target and then collected by the electro-optical receiver. Range to the target is determined by measuring the round-trip time of the electromagnetic impulse. Radial velocity of the target is measured by either determining the Doppler shift of the emitted wavelength or by performing multiple range measurements and calculating the rate of change in range. Similar to radar, the intensity and profile of LIDAR reflected signals vary with the beam wavelength and with the reflectance characteristics of the surface reflecting the beam. LIDARs can be categorised according to the type of measurement, the detection technique, the type of laser and operational wavelength, the type of interferometer employed in a coherent laser radar (where applicable), the modulation technique, the demodulation technique, the purpose, the type of data collected, or the data format. In addition, laser radars can be classed as monostatic or bistatic, depending on whether the receiver and the emitter are collocated or not. The different types of lasers adopted for LIDAR systems and their respective carrier wavelengths summarized in Table 1 [11]. The techniques employed for LIDAR are identified in Table 2 and, its functions and measurements used are summarised in Table 3 [12]. The appellation is seldom sufficient to completely identify what it does and does not define the performance characteristics. The versatility of lasers is evident from their available variety. Wavelength-dependent technological limitations frequently 
This is the author pre-publication version. This paper does not include the changes arising from the revision, formatting and publishing process. The final paper that should be used for referencing is:

R. Sabatini, M. A. Richardson, A. Gardi, S. Ramasamy, "Airborne laser sensors and integrated systems", Progress in Aerospace Sciences (2015), http: //dx.doi.org/10.1016/j.paerosci.2015.07.002i.

prevent simple parametric extrapolation of performance from one type of system to another. These limitations make routine performance at one laser wavelength well beyond the state-of-theart at another wavelength. Due to the physical principles governing laser emission, tuneability both in terms of wavelength and in terms of power are very difficultly introduced. Passive optics and conventional radars - Radio Frequency (RF) through Millimeter-Wave (MMW) on the other hand are natively capable of large tuneability in their design without major changes in technology.

Table 1

LIDAR types.

\begin{tabular}{ll}
\hline Types of Lasers (Typical) & Carrier Wavelength \\
\hline $\mathrm{CO}_{2}$ & $9.2 \mu \mathrm{m}-11.2 \mu \mathrm{m}$ \\
Er:YAG & $2 \mu \mathrm{m}$ \\
Raman Shifted Nd:YAG & $1.54 \mu \mathrm{m}$ \\
Nd:YAG & $1,06 \mu \mathrm{m}$ \\
GaAlAs & $0.8 \mu \mathrm{m}-0.904 \mu \mathrm{m}$ \\
$\mathrm{HeNe}$ & $0.63 \mu \mathrm{m}$ \\
Frequency Doubled Nd:YAG & $0.53 \mu \mathrm{m}$ \\
\hline
\end{tabular}

Table 2

LIDAR techniques.

\begin{tabular}{lll}
\hline $\begin{array}{l}\text { Detection } \\
\text { Technique }\end{array}$ & $\begin{array}{l}\text { Interferometer } \\
\text { Type }\end{array}$ & $\begin{array}{l}\text { Modulation } \\
\text { Technique }\end{array}$ \\
\hline Direct Detection & Not Applicable & $\begin{array}{l}\text { Pulsed Amplitude } \\
\text { Modulation (AM) }\end{array}$ \\
Coherent Detection & Heterodyne & $\begin{array}{l}\text { Pulsed Amplitude } \\
\text { Modulation (AM) }\end{array}$ \\
& Homodyne Offset & $\begin{array}{l}\text { Frequency } \\
\text { Homodyne }\end{array}$ \\
& & $\begin{array}{l}\text { Modulation (FM) } \\
\text { Hybrid (AM/FM, } \\
\text { Pulse Burst) } \\
\end{array}$ \\
& & None (CW) \\
\hline
\end{tabular}

Table 3

LIDAR functions and measurements.

\begin{tabular}{ll}
\hline Functions & Measurements \\
\hline Tracking & Amplitude (Reflectance) \\
Moving Target Indication (MIT) & Range (Time delay) \\
Machine Vision & Velocity (Doppler Shift or \\
Velocimetry & Differential Range) \\
Wind Shear Detection & Angular Position \\
Target Identification & Vibration Spectra \\
Imaging & \\
Vibration Sensing & \\
\hline
\end{tabular}

\subsection{Receiver detection techniques}

Direct and coherent detection types are the two types of LIDAR systems. In direct detection laser radar (Fig. 1), the inbound radiation is focused onto a photosensitive element generating a voltage (or current) that is directly proportional to the incident energy. This process is analogous to conventional passive optical receivers.

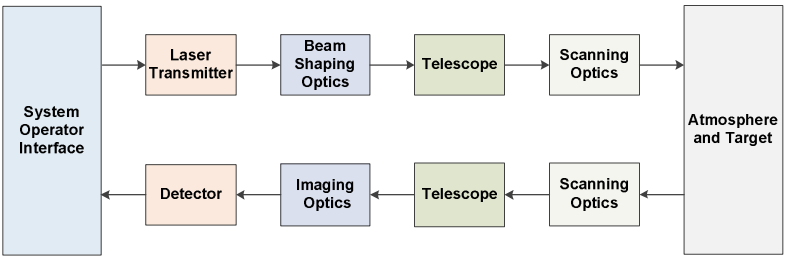

Fig. 1. Block diagram of a direct detection LIDAR.

A block diagram, of heterodyne (coherent) detection LIDAR is shown in Fig. 2. An optical signal is generated by the laser emitter. The divergence and diameter of the laser beam are then adjusted when necessary to the rest of the system by beamshaping optics. In a monostatic system, the transmitted laser signal enters a Transmit-to-Receive (T/R) switch. The T/R switch permits the LIDAR transmitter and receiver to operate through a common optical aperture. The LIDAR signal then enters the beam expander or output telescope and the scanning optics that direct the optical signal to the target. In a monostatic system, radiation reflected from the target is collected by the scanning optics and the beam expander, which now acts as an optical receiver. The $\mathrm{T} / \mathrm{R}$ switch directs the received radiation to an optical mixer, where it is combined with an optical reference signal generated by the local oscillator. The combined signal is then focussed onto a photosensitive detector by the imaging optics. The photosensitive detector generates an electrical signal in response to the received optical signal. The electrical signal is then high-pass filtered to remove any low-frequency components, such as those from background sources and from the local oscillator-induced dc signal. The high frequency components of this electrical signal contain the target information, which is then extracted from the electrical signal by signal and data processors.
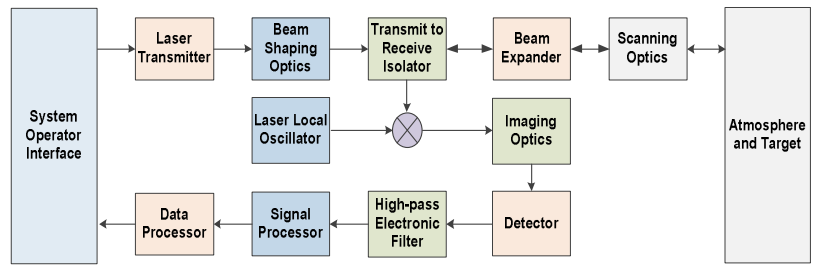

Fig. 2. Block diagram of a coherent detection LIDAR.

In a bistatic system, the $\mathrm{T} / \mathrm{R}$ switch is omitted. An additional distinction between conventional heterodyne and homodyne receivers is that while the former requires a separate laser source to serve as the local oscillator the latter uses the transmitter source for laser radiation as the local oscillator for the receiver. Offset homodyne receivers have also been developed, in which the local oscillator beam portion is frequency shifted from the transmitter beam.

\subsection{Laser range finders}

Operational range finders were introduced as early as the midsixties after the initial development by John D. Myers, only five years after Theodore Maiman presented the first working laser. Since then, a number of Laser Range Finders (LRF) and Laser Target Designators (LTD) have been manufactured in many countries all over the world. The high radiance and collimation of lasers makes it possible to determine distances with great accuracy. The accurate range and angle information provided by the LRF employed in modern Fire Control Systems (FCS) is responsible for a major advance in the precision and effectiveness of weapons in battlefield conditions. A variety of laser technologies have been applied to rangefinders and NeodymiumYttrium Aluminium Garnet (Nd:YAG) LRF, operating at a 
This is the author pre-publication version. This paper does not include the changes arising from the revision, formatting and publishing process. The final paper that should be used for referencing is:

R. Sabatini, M. A. Richardson, A. Gardi, S. Ramasamy, "Airborne laser sensors and integrated systems", Progress in Aerospace Sciences (2015), http: //dx.doi.org/10.1016/j.paerosci.2015.07.002i.

wavelength of $1064 \mathrm{~nm}$ and based on the principle of pulse timeof-flight measurement, are the state-of-the-art. LRF based on Er:fiber and Raman-shifted Nd:YAG lasers are used in cases where eye-safety is fundamental. $\mathrm{CO}_{2}$ eye-safe LRF, operating at $10.6 \mu \mathrm{m}$, have been developed in many configurations and they can play a significant role in conjunction with passive thermal imaging systems and other multi-functional system applications. The architecture of a typical LRF system transmitter and receiver is shown in Fig. 3 and Fig. 4 respectively [12].

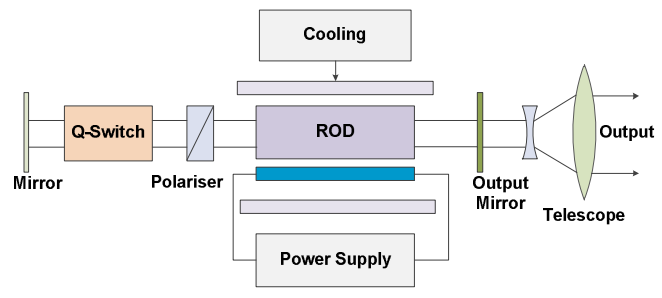

Fig. 3. Typical LRF transmitter.

The transmitter contains an electro-optically Q-switched laser, while the radiation scattered from the target is collected by the receiver, which may be a conventional mirror or lens system. The beam divergence from the laser may be several milliradians and in order to obtain accurate target definition a simple collimating telescope has been added, which would reduce this to less than 1 $\mathrm{mrad}$. The receiver may also incorporate a narrow pass-band spectral filter centred on the laser wavelength to further reduce the standing background signal which contributes to the overall system noise. The receiver electronics are illustrated in Fig. 4 and typically include: an analog section, which amplifies the return pulse whilst retaining its shape and a digital section, which performs logical timing processes and calculates the range.

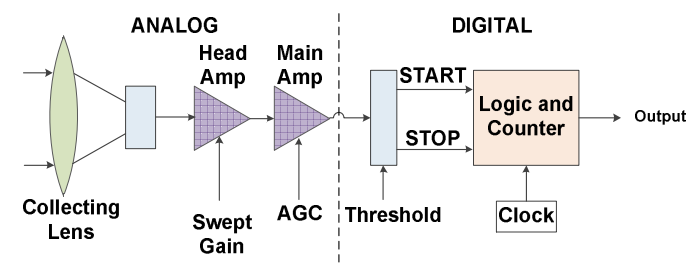

Fig. 4. Typical LRF receiver.

\subsection{Airborne lasers in multi-sensor systems}

LIDAR, while being a powerful sensor for airborne applications, still has its limitations. As an obstacle warning or an attack sensor its range is degraded by adverse weather. When used for terrain flying, its narrow beam width does not allow wide coverage as a conventional radar will do. However, as part of a multi-sensor system, LIDAR can be very effective. An example is the combination of LIDAR with a Forward Looking Infra-Red (FLIR) sensor in a single system. This solution provides night vision capability using the FLIR with the terrain flying and obstacle avoidance capabilities of the laser. Fig. 5 is an example of a combined radar and electronic surveillance system which could perform five different functions:

- passive listening, for gathering intelligence on other radar and transmissions over a wide frequency band;

- laser obstacle avoidance and terrain following for covert operations;

- primary radar for use in bad weather or as a cueing system for LIDAR;
- passive detection for accurate determination of the bearing of jammers or other radars;

- real-time passive night vision.

The control of these functions involves complex computer processing responding to the various operational situations. Considerable research is currently underway addressing the problem of laser sensors integration in airborne nav-attack, reconnaissance and electronic warfare systems. Data fusion algorithms using Extended Kalman Filter (EKF), Unscented Kalman Filter (UKF) and particle filter have been developed for augmenting measurements from various sensors including LIDAR and FLIR [13]. An Automatic Target Recognition (ATR) system for use in air-to-air homing missiles that recognises and tracks a target through fusing data from LIDAR and Forward Looking Infrared (FLIR) sensors is presented in [14]. In this architecture, LIDAR and FLIR complement each other in their sensing characteristics and offer a robust decision design. EKF based data fusion involving information from LIDAR, visual sensors, FLIR and acoustic sensors are used for $2 \mathrm{D}$ and $3 \mathrm{D}$ imagery to enable aircraft to autonomously detect and prosecute targets in uncertain complex adversarial environments have been developed [15]. In-flight fusion of hyperspectral imaging and waveform LIDAR for three-dimensional studies of ecosystems have been developed in the recent past [16].

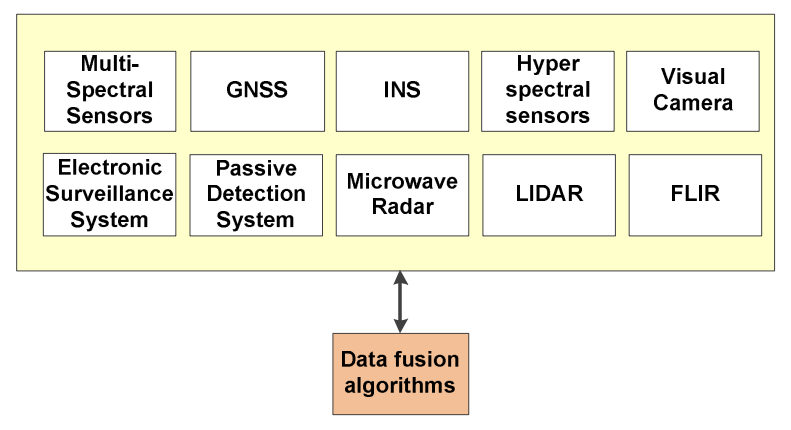

Fig. 5. Multi-sensor system.

\subsection{Civil applications}

There has been a substantial growth in commercial use of airborne lasers for a variety of applications ranging from remote sensing to archaeological surveying.

\subsubsection{LIDAR remote sensing}

Airborne LIDAR systems are increasingly used in remote sensing applications including digital elevation mapping, canopy height retrieval, forest monitoring and Earth sciences. Airborne LIDAR systems are capable to operate at all times and during all-weather conditions [17]. LIDAR is widely used for generating highresolution topographic data including Digital Elevation Models (DEM), Digital Terrain Model (DTM) and Digital Surface Model (DSM) and provides vertical accuracies of 15 to 100 centimeters. The topographic information provided by LIDAR is critical for engineering tasks including transportation (rail/road planning), mining reclamations, urban and rural planning, hydrology and coastal erosion management, corridor mapping, landside analysis, geological studies, disaster monitoring, telecommunication cell network planning, and natural resource assessments (coal stack monitoring) [18]. LIDAR altimeters can also estimate the key features of canopy structures very efficiently and accurately. Airborne LIDAR systems can directly measure the horizontal and 
This is the author pre-publication version. This paper does not include the changes arising from the revision, formatting and publishing process. The final paper that should be used for referencing is:

R. Sabatini, M. A. Richardson, A. Gardi, S. Ramasamy, "Airborne laser sensors and integrated systems", Progress in Aerospace Sciences (2015), http: //dx.doi.org/10.1016/j.paerosci.2015.07.002i.

vertical distribution of vegetation down through the canopy profile [5], providing three-dimensional characterizations of internal vegetation structure below the canopy [19,20]. Many studies point out that the tree and canopy height precision obtained from LIDAR systems is actually superior to traditional field inventory methods [21, 22]. Airborne laser scanning inventories can be particularly advantageous in situations where little ground based inventory exists and the forest site access is limited because of poor infrastructure or remote terrain [23]. Airborne LIDAR systems offer great flexibility in data capture, high speed of data delivery, better elevation accuracy in difficult terrain, ease of Geographic Information Systems (GIS) integration, high sampling densities and high degree of automation [4, 24]. Consequently, airborne LIDAR systems are now employed in most regions of the world for the remote sensing of forest areas [25]. A typical example of airborne laser based remote sensing is illustrated in Fig. 6 .

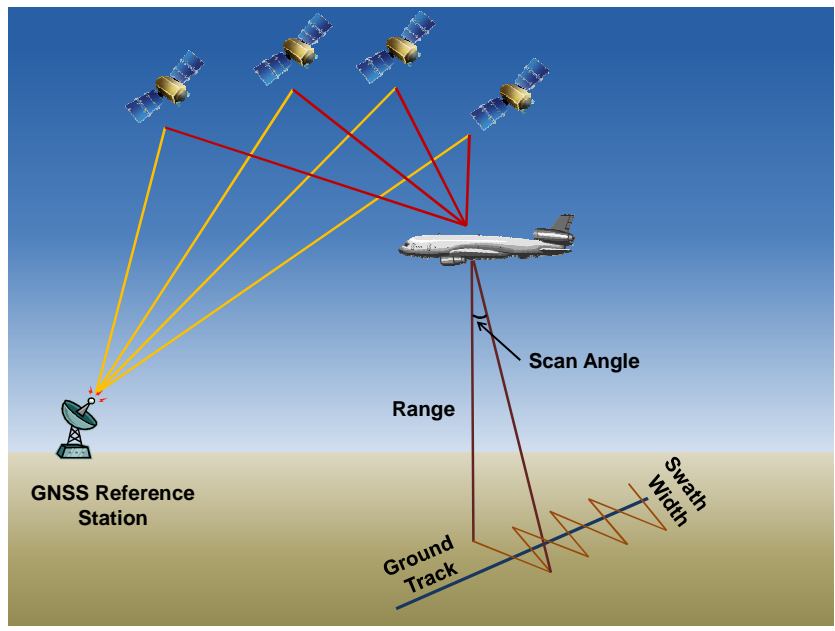

Fig. 6. Airborne laser based remote sensing.

A combination of very accurate GNSS and airborne LIDAR systems are used in geology and seismology applications for detecting faults and measuring uplift. Lasers have made it possible to directly measure the distance from ground tracking stations to the Earth orbiting satellites that are equipped with optical retro-reflectors. Analogous measurements have been exploiting retro-reflecting arrays placed on the Moon by US astronauts and unmanned USSR lunar rovers to accurately monitor the distance from our natural satellite. Lunar Laser Ranging (LLR) was technologically challenging, expensive and operationally limited for a long time due to the Moon's relatively large distance from the Earth. Currently, LLR is primarily used for testing general relativity, detecting changes in the universal gravitational constant and for lunar science, and are also increasingly used for geoscience applications [26, 27]. Satellite Laser Ranging (SLR) [28] offers remarkable geodetic measurement accuracies by exploiting satellites in complementary orbits, at different altitudes and inclinations. The equipment for SLR observing units are smaller, less expensive and transportable, allowing them to be upgraded more frequently to exploit technological advances.

\subsubsection{LIDAR obstacle detection and avoidance systems}

Lasers have also found applications in helping to solve the problems of very low level flight, especially by military aircraft and Remotely-Piloted Aircraft System (RPAS). In order to achieve mission effectiveness in the present threat environment, military aircraft operations have focused on-terrain or nap-of-theearth flying. However, flight at very low levels greatly increases the probability of striking the terrain or man-made obstacles such as wires, poles, towers or buildings. Conventional radar has the capability to provide a terrain following mode; however, it is inadequate for development into a reliable Obstacle Warning System (OWS). For a LIDAR to be effective as a terrain following and obstacle avoidance system it must meet certain operational criteria of performance, which dictate the system design. For an OWS to be effective it must meet certain requirements. The most important requirement is reliable detection of all obstacles (terrain masses, buildings, poles, towers and power cables) at almost all angles of incidence of radiation with a very high probability of detection and very low false alarm rate. The need for a high probability of detection is crucial since no obstacle must go undetected. A low false alarm rate is required to prevent spurious warnings that would increase the flight crew's workload, cause it to unnecessarily increase the altitude, and thus making the aircraft more detectable. Another operational requirement is a high minimum detection range. The required minimum detection range value depends upon the aircraft speed, vertical manoeuvring capability (i.e., very different for helicopter and for airplane platforms), and pilot reaction time. As an example, for an airplane flying straight and level at $300 \mathrm{~m} / \mathrm{s}$ and allowing a reasonable pilot reaction time and aircraft response time of between five to ten seconds, detection ranges of about two to three kilometres are adequate. Laser Obstacle Avoidance and Monitoring (LOAM) system have been developed for helicopters and RPAS [29-32] and it performs echo detection through analogue signal processing that comprises an optical-electrical conversion, a signal pre-amplification and a threshold comparison. Local analysis and global analysis are performed for reliable obstacle data processing. The local analysis is performed on single echoes in order to determine range, angular coordinates and the characteristics of the obstacle. The global analysis processes groups of echoes detected over a scan period, with the related information provided by the local analysis process, in order to reconstruct the shape and determine the type of obstacles. LOAM is capable of automatically classifying obstacles according to the following classes: wires, poles/trees or extended obstacles. Information relative to the detected obstacles can be provided on a dedicated display whose screen represents the Field of View (FOV) of the system. The processing unit estimates the future trajectory, calculates the intersections with obstacles and determines the optimal avoidance trajectories. An example of an integrated LOAM architecture is illustrated in Fig. 7. 
This is the author pre-publication version. This paper does not include the changes arising from the revision, formatting and publishing process. The final paper that should be used for referencing is:

R. Sabatini, M. A. Richardson, A. Gardi, S. Ramasamy, "Airborne laser sensors and integrated systems", Progress in Aerospace Sciences (2015), http: //dx.doi.org/10.1016/j.paerosci.2015.07.002i.

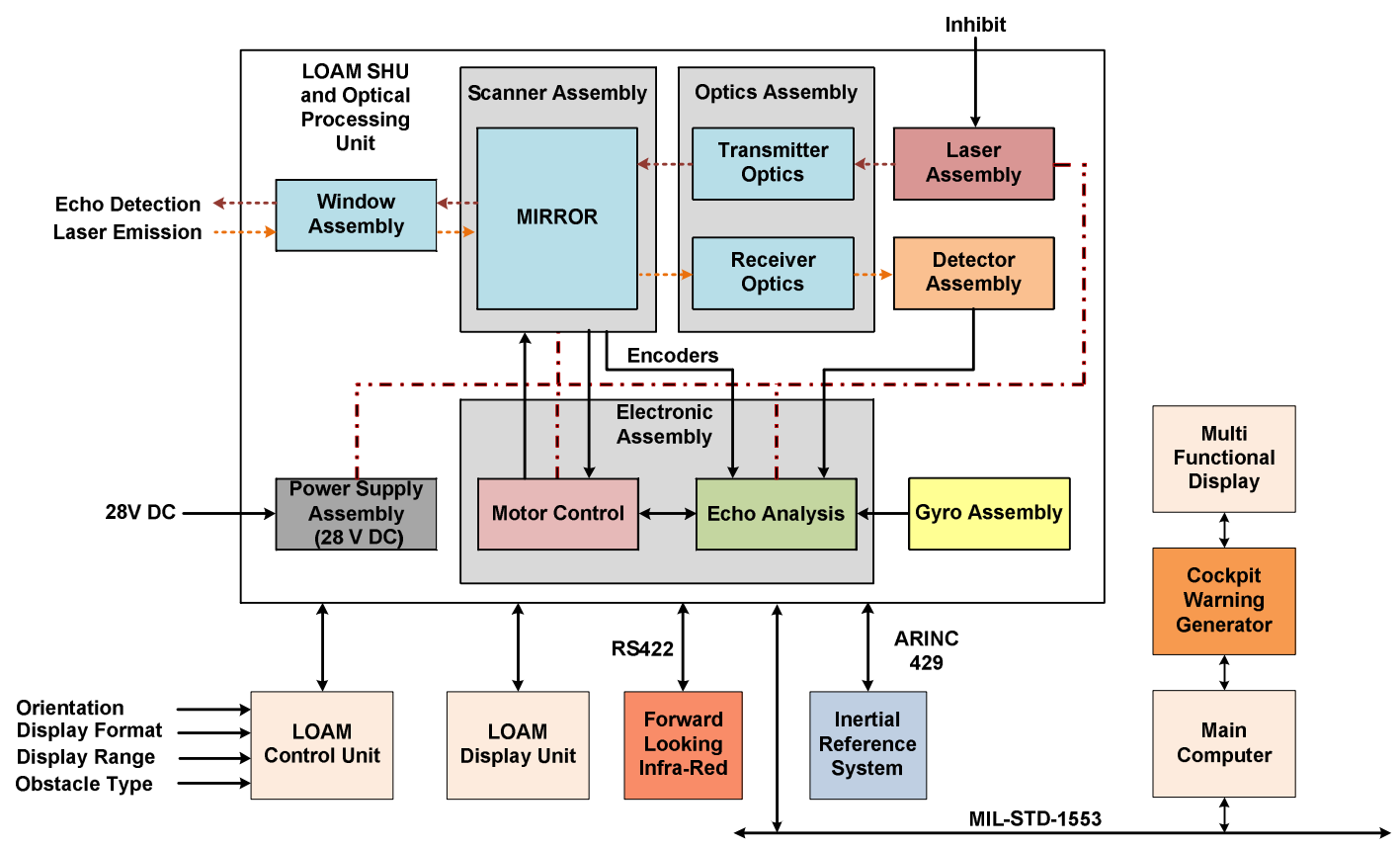

Fig. 7. LOAM system architecture.

\subsubsection{Differential absorption LIDAR}

The adoption of LIDAR for atmospheric soundings originated in the 1960's as an evolution of radar meteorology to determine the atmospheric composition including clouds and aerosols. Similarly to the absorption spectroscopy, these sensors measure the transmittance on the specific spectral lines associated with transitions in the vibrational state of the molecule and compare them with the transmittance outside the vibration spectral lines. The fraction of the two measures $R_{O N / O F F}$ is expressed as:

$$
\begin{gathered}
R_{O N / O F F}=\frac{E\left(\lambda_{O N}\right)}{E\left(\lambda_{O F F}\right)}=\frac{\tau_{O N}}{\tau_{O F F}} \\
R_{O N / O F F}=e^{-\left[\psi_{C S}\left(\lambda_{O N}\right)-\psi_{C S}\left(\lambda_{O F F}\right)\right] \int_{0}^{D} n_{C S}(r) d r}
\end{gathered}
$$

where $\psi_{C S}$ is the molecular cross-section of the Chemical Species (CS), $\lambda_{O N}$ is the wavelength of the absorption spectral line, $\lambda_{O F F}$ is the wavelength of the off-absorption spectral line, $\tau$ is the transmittance, $n_{C S}$ is the concentration of the chemical species and $D$ is the total beam length. The technique is referred to as Differential Absorption LIDAR (DIAL). The advent of powerful laser sources and pointing systems, with low weight and packaged in relatively small casings, allows for different DIAL systems to be installed on aircraft, satellites or other aerial/surface vehicles. LIDAR for meteorological applications was initially based on ruby lasers. The most widely used DIAL configurations for atmospheric sounding are based on active monostatic configurations, measuring both the elastic backscatter generated along the outward path and the radiance of the illuminated Earth surface towards the airborne system. Some effects, like the variation of turbulence structure constants, are difficult to model in these techniques, and any mathematical extrapolation or empirical estimation would introduce considerable uncertainties in the measurements. LIDAR measurements are accurate but limited to shorter ranges than those provided by imaging sensors. Atmospheric turbulence introduces significant fluctuations of laser energy on the focal plane, and some nonlinear propagation effects, such as bleaching and thermal blooming, may also cause severe attenuations of laser beams propagating in the atmosphere
[12, 33, 34]. Additionally, due the advent of powerful tuneable lasers, a variety of DIAL systems have been developed for measuring the concentration/column density of various important molecular species, including carbon oxides $\left(\mathrm{CO}_{\mathrm{X}}\right)$ and other Volatile Organic Compounds (VOC), nitrogen oxides $\left(\mathrm{NO}_{\mathrm{X}}\right)$, ozone $\left(\mathrm{O}_{3}\right)$, both locally and over extended geographic areas [12, 35-55].

\subsubsection{Tuneable laser absorption spectroscopy}

Absorption Spectroscopy is a family of in situ techniques that determines a trace gas concentration by measuring the intensity of the absorption effects on specific spectral lines associated with a transition in the vibrational state of that gas molecule [56]. Tuneable Laser Absorption Spectroscopy (TLAS) employs lasers to irradiate an absorption cell at the absorption spectral line of the selected gas molecule. A number of airborne TLAS systems were employed for the in situ measurement of various pollutants in the last two decades [57-68]. The technique was originally based on lead-salt diode laser sources emitting in the Mid-InfraRed (MIR), and subsequently adopted solid-state semiconductor sources [69]. The emergence in more recent times of Quantum Cascade Lasers (QCL) with operational wavelengths ranging between $2.6 \mu \mathrm{m}$ to beyond $300 \mu \mathrm{m}$ is having substantial impacts on TLAS [56, 6973], and several QCL-based TLAS applications are emerging [7479]. Single-Particle Light Scattering was also implemented in airborne platforms to measure size distribution of cloud droplets

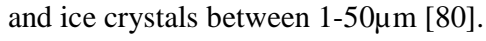

\subsubsection{Laser turbulence detection system}

Conventional Doppler radars have been experimented for decades to study atmospheric phenomena such as convective cloud dynamics, boundary layer kinematics, and turbulence properties [81]. Most meteorological radars operate at wavelengths between 3 and $10 \mathrm{~cm}$, therefore they may only detect particles of the order of a few hundred microns in diameter, such as water droplets and hailstones, and as such they can only detect severe storms, and are of little use for studying atmospheric dynamics in clear-air. The laser Doppler radar, also referred to as Doppler LIDAR proves to 
This is the author pre-publication version. This paper does not include the changes arising from the revision, formatting and publishing process. The final paper that should be used for referencing is:

R. Sabatini, M. A. Richardson, A. Gardi, S. Ramasamy, "Airborne laser sensors and integrated systems", Progress in Aerospace Sciences (2015), http: //dx.doi.org/10.1016/j.paerosci.2015.07.002i.

be a more capable and versatile remote sensor, also enabling the measurement of clear-air wind velocities [49, 82]. The Doppler LIDAR sensing technique methods of sensing wind velocity using Doppler LIDAR are based on the assumption that aerosols are fully entrained in the air mass motion caused by the wind. The very narrow spectral and geometrical laser beam widths provide sufficient backscattered radiation to permit measurement of very low velocities by means of heterodyne detection. This process is termed Laser Doppler Velocimetry (LDV) [83, 84]. Doppler LIDAR systems have been employed for some time and the principal aim initially was to provide a system to perform remote measurements of the wind so that very large changes (e.g., wind shear) could be identified. Modern Doppler LIDAR systems can be fitted into the aircraft nosecone and can be used to measure the wind velocity in the frontal region, unaffected by the aerodynamic blockage due to fuselage or the wing. Currently, research is addressing the design of systems exploiting wind velocity and variations measurements by airborne LIDAR systems to enhance the autopilot and autothrottle control logics, reducing the pilots' workload. These system evolutions could be deployed on both military and civilian aircraft to improve safety, particularly during take-offs and landings. These delicate flight phases can in fact be affected very seriously by wind shear, and its most severe form, known as microburst, which have caused several aircraft accidents. Microburst last for a short period of time, about 15 minutes, and occur over a distance of about three to four kilometres. Fig. 8 (a) illustrates the wind shear phenomenon affecting an aircraft in the critical phase of final approach and Fig. 8 (b) illustrates a final approach flown by an aircraft equipped with a Doppler LIDAR system for wind velocity detection in its nosecone with a suitably adopted autopilot for employing the required control loop.

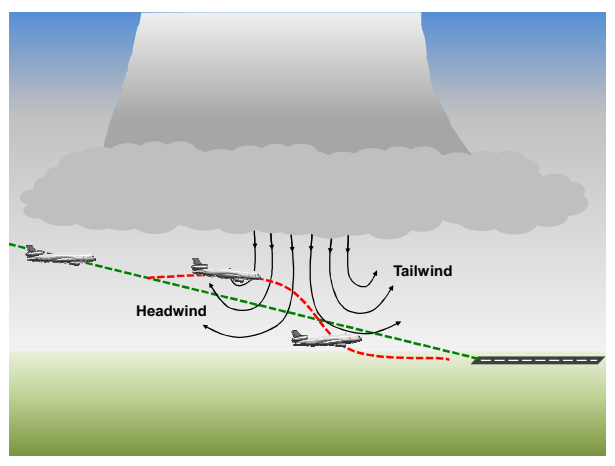

(a)

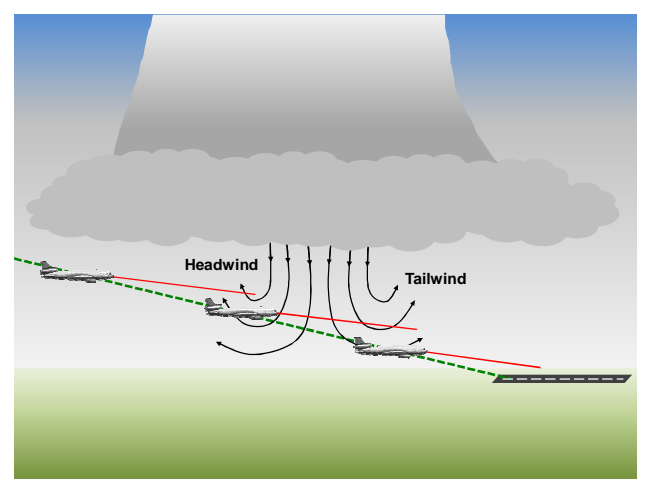

(b)

Fig. 8. Illustration of the wind shear phenomenon affecting an aircraft in the critical phase of final approach (a), and a final approach flown by an aircraft equipped with a Doppler LIDAR system for wind velocity detection in its nosecone (b).
The danger lays in the fact that very significant variations in the aircraft dynamical state can be experienced in case of wind shear during approach and initial climb, and in these conditions the aircraft lacks the necessary altitude to perform a safe recovery. When flying through a microburst, the aircraft initially encounters a strong head wind, increasing its airspeed and lifting it above its correct path. Human pilots and autopilots are therefore led to reduce engine thrust to rectify the flight profile. Within a few seconds the aircraft encounters a sudden downdraft and strong tail winds, diminishing its airspeed and increasing its descent rate. The recovery action involving an increase in engine thrust may not happen quickly enough to avoid an impact with the terrain.

\subsubsection{Landslide monitoring}

The possibility to directly acquire an accurate and high density 3D point cloud has made LIDAR the preferred technology for topographic data collection in forestry areas [85-87]. A typical LIDAR system used for landslide monitoring purposes consists of a laser ranging and scanning unit, together with a Position and Orientation System (POS), which encompasses an integrated Differential Global Positioning System (DGPS) and an Inertial Navigation System (INS) [88]. The laser ranging system measures the distances from the sensor to the mapped terrain, while the onboard GPS/INS integrated navigation system provides the position, velocity and attitude information of the platform. LIDAR data collection is car- ried out in a strip-wise fashion and the ground coordinates of the laser footprints are obtained [5]. In spite of very dense and precise spatial data, these systems are rather poor in spectral sensitivity. In order to overcome this problem, hyperspectral datasets are employed [89]. These systems are independent and consist of a hyperspectral sensor head, a miniature GPS/INS sensor, a data acquisition unit in a rugged PC with display unit and power supply. Innovative integrated airborne and wireless systems for landslide monitoring have also been developed in the recent years [90]. A typical airborne laser scanning process for obtaining landslide maps based on statistical and GIS analysis is depicted in Fig. 9.

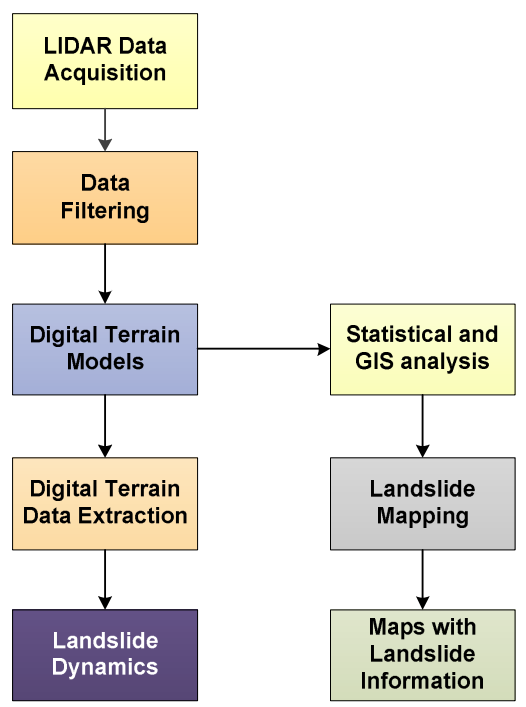

Fig. 9. Airborne laser scanning for landslide monitoring. Adapted from [67].

The key advantages of adopting airborne laser systems for landslide monitoring are: 
This is the author pre-publication version. This paper does not include the changes arising from the revision, formatting and publishing process. The final paper that should be used for referencing is:

R. Sabatini, M. A. Richardson, A. Gardi, S. Ramasamy, "Airborne laser sensors and integrated systems", Progress in Aerospace Sciences (2015), http: //dx.doi.org/10.1016/j.paerosci.2015.07.002i.

- Uses of an active sensor in the form of a narrow laser beam with high penetration capability, making the measurements independent of weather conditions and time of a day.

- The high penetration rates of laser signals in densely vegetated (wooded) areas (up to $70 \%$ ) assure that sufficient reflections come from the terrain surface [4]. In such areas most of the traditional measurement techniques fail.

- Obtained data are characterized by the high density, even tens of points per square meter with high vertical precision from about 0.1 to $0.3 \mathrm{~m}$.

\subsubsection{Bathymetry}

Methods have been developed to measure, inventory, and predict different hydrological variables such as water-surface ex- tent and temperature, water volume, velocity of waves, organic and inorganic water constituents, or water depth, known as bathymetry. Using this technique, a three dimensional description of the ground profile is obtained including sea and river beds. In contrast to topographic laser scanners operating with near infrared lasers, bathymetric sensors make use of green lasers [71]. Unlike infrared lasers, these lasers are able to penetrate the water column and thus may reach the sea bed. The measuring depth is limited due to attenuation of the laser energy by absorption, scattering, and refraction effects while the laser pulse is traveling through the water column. The current generation of Airborne Laser Bathymetry (ALB) scanner systems utilise very short and narrow green laser pulses. The principle of operation is that the airborne laser pulse interacts with the three components of shallow water bodies: the water surface, the water column and the benthic or bottom region. In general, a water body is considered as a complex mix of particles and various dissolved substances that, in combination with a specific bottom surface yield optical properties that can vary over a large continuum of values [91]. Several laser sources were analysed and it was found that a green pulse at $532 \mathrm{~nm}$ is very well suited for all ALB applications [92, 93]. This laser wavelength is the frequency-doubled version of the conventional $1064 \mathrm{~nm}$ laser [94] and it also has the capability to maximally penetrate different water bodies and to return an echo to the airborne receiver. For example, the EAARL (Experimental Advanced Airborne Research LIDAR) is an airborne system that provides capabilities to simultaneously survey multiple heterogeneous marine and terrestrial environments including coral reefs, near shore benthic habitats, coastal vegetation, and shallow braided river systems $[95,96]$.

\subsubsection{Laser gated television}

There are two basic types of laser television systems using laser sources to obtain images from airborne platforms. These systems implement line-scan and gated television methods. In the first method a narrow laser beam is scanned over the ground and the return radiation is measured by a spectrally filtered optical detector onboard the aircraft. In a gated TV system the whole scene is illuminated by a short pulse of laser radiation and the image is recorded through a conventional optical system. However, in this case, the image tube is gated such that it only records the return optical image after a finite delay time that is determined by the range to the target area. The line-scan and gated-TV systems offer both complimentary and unique capabilities compared with normal photography or microwave radar. For example, compared to normal photography, the linescan system can operate on a 24 hour basis since it supplies its own source of illumination. Side- looking microwave radar also has a full-time capability and has a much larger area of coverage. However, the limiting resolution is far superior for laser systems, which is very important in certain applications. In the case of the gated-TV system, again the night- time capability has marked advantages over photography, and in addition, the gating facility allows penetration of haze when nor- mal visibility is poor. All these attributes offer distinct advantages for many forms of reconnaissance applications, although the spe- cific gains are dependent upon the mission $[97,98]$.

\subsubsection{D building reconstruction}

Since the late 1990 's, new data sources, such as high-resolution images and airborne laser scanning datasets, became available that required the development of more advanced algorithms. These new approaches continue to be studied as new sensor technology with higher resolution and accuracy are developed. Chief among the current day technology is Light Detection And Ranging (LIDAR), which has been successfully used for this field of study over the past two decades. While most existing approaches show promising results toward automatic generation of $3 \mathrm{D}$ building models, a number of issues remain to be addressed. The main objective of this research was to reconstruct 3D building models from airborne laser scanning (ALS) data. To achieve this objective, a complete framework for this process, including LIDAR filtering, building extraction, roof plane segmentation, and 3D reconstruction has been proposed [99-102].

\subsection{Military applications}

\subsubsection{Directed energy weapons}

The first airborne Directed Energy Weapon (DEW) was tested in the mid-1970's, under a program called the Airborne Laser Laboratory (ALL) [103]. The tested laser was a gas dynamic $\mathrm{CO}_{2}$ system (400 kW version) developed by AVCO Everett and, later, by United Technologies in the U.S. The ALL system was installed on aKC-135 aircraft, along with an elaborate beam director. The pro- gram was technically successful and the ability of the system to shoot down missiles in flight was demonstrated [104]. In the late 1970s, further programs funded by the U.S. Navy convinced that the threat of missiles launched against a flotilla of ships could be minimised by ship borne DEW's. These efforts resulted in the Mid-Infrared Advanced Chemical Laser (MIRACL) [105,106], developed by TRW, and the Sea Lite Beam Director (SLBD) developed by Hughes. However, by the time MIRACL was operational, ship borne antimissiles and guns proved to be a more attractive choice due to their lower cost and more mature technology. If DEW's were too expensive and complex for the defence of tactical assets, then the logic followed was that they might be used to "take out" opponent's strategic military assets, such as satellites. Although these early airborne and non-airborne applications did not reach the final operational stage, they served to develop several different laser technologies, which are now being used in current military developments and in a wide diversity of commercial applications. DEWs are generally developed based on gas discharge (oxygen- iodine and $\mathrm{CO}_{2}$ ) and chemical (deuterium fluoride) lasers. An air- borne laser DEW system developed in the U.S. is the Air Borne Laser (ABL), which uses a high-energy laser mounted on a modified 747-400F (the Boeing YAL-1) aircraft to shoot down heater ballistic missiles in their boost phase [103, 107, 108]. Particularly, a tracking laser beam illuminates the missile, and computers measure the distance and calculate its course and direction. After acquiring and locking onto the target, a second laser (with weapons- class strength) fires a 3- to 5-second burst from a turret located in the 747 nose. The missile is then destroyed over the launch area. The airborne laser fires a Chemical Oxygen Iodine 
This is the author pre-publication version. This paper does not include the changes arising from the revision, formatting and publishing process. The final paper that should be used for referencing is:

R. Sabatini, M. A. Richardson, A. Gardi, S. Ramasamy, "Airborne laser sensors and integrated systems", Progress in Aerospace Sciences (2015), http: //dx.doi.org/10.1016/j.paerosci.2015.07.002i.

Laser (COIL), which was invented at Phillips Lab in 1977. The laser fuel consists of the same chemicals found in hair bleach and Drano (hydrogen peroxide and potassium hydroxide) which are then combined with chlorine gas and water. The laser operates at an infrared wavelength of 1.315 microns, which is invisible to the eye. By recycling chemicals, building with plastics and using a unique cooling process, the COIL team was able to make the laser lighter and more efficient while, at the same time, increasing its power by 400 percent in five years. The flight-weighted ABL module is similar in performance and power levels to the multihundred kilowatt class COIL Baseline Demonstration Laser (BDL-2) module demonstrated by TRW. Performance requirements for the airborne laser weapons system were established by the operational scenarios and support requirements defined by the user, U.S. Air Combat Command, and by measured target vulnerability characteristics provided by the U.S. Air Force lethality and vulnerability community centred at the Phillips Laboratory. During its inception, the modified Boeing 747 was only able to carry enough fuel to fire the weapon at most 40 times. The US Naval Research Lab worked towards developing a similarly powerful megawatt-class free electron laser, which will be able to cut through 20ft of steel per second. The ABL Program Definition and Risk Reduction (PDRR) phase was supported by a robust technology insertion and risk reduction program to provide early confidence that scaling to Engineering and Manufacturing Development (EMD) program performance is feasible. The technology and concept design efforts provided key answers to the PDRR design effort in the areas of lethality, atmospheric characterization, beam control, aircraft systems integration, and environmental concerns. In 2001, the ABL development program was transformed to an acquisition program under the Missile Defense Agency (MDA). In 2011, the ABL Test (ABLT) program was successful in demonstrating the capability of shooting down a number of potential targets using a high power directed energy weapon. A pictorial representation of the ABL equipped aircraft is shown in Fig. 10. The Advanced Tactical Laser (ATL) programme was initiated to adopt the technologies deveoped for the ABL, providing a cheaper and smaller system, suitable for carriage on aircraft such as the AC130 Spectre or V-22 Osprey and to be used as a close air support weapon. In January 2006, U.S. Air Force's 46th Test Wing provided Boeing with a $\mathrm{C}-130 \mathrm{H}$ Hercules for trials of the prototype weapon, claimed to be in the Mega Watt class, using COIL technology. The laser prototype was mounted on the aircraft to perform lethality trials against a range of ground targets. The laser was successfully fired in 2008 [109]. The Boeing Laser Avenger (BLA) is an infrared laser system mounted on an AN/ TWQ-1 Avenger combat vehicle developed by Boeing Combat Systems in the United States [110]. Northrop Grumman is currently developing a high-energy solid-state laser weapon system called FIRESTRIKE (Joint High-Power Solid-State Laser - JHPSSL), which was publicised in 2008. This modular system uses $15 \mathrm{~kW}$ and can be integrated to provide various levels of power. The Beriev A-60 is a Russian laser direct energy laboratory aircraft developed based on the Ilyushin Il-76MD transport aircraft [111]. Observable design modifications to the Il76MD Candid host airframe included:

- Nose mounted fairing for the installation of a steerable beam director turret (similar arrangement to the much later Boeing YAL-1A design).

- Removal of aft clamshell doors, replacement shell with a large axial exhaust aperture.

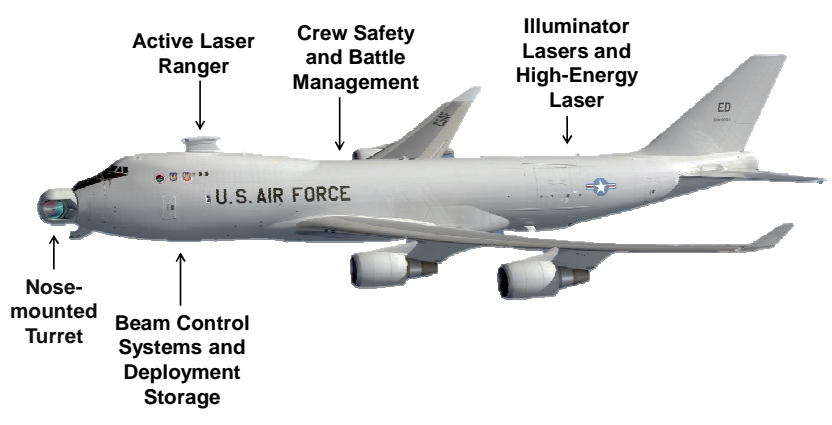

Fig. 10. ABL concept of operation - Modified 747- 400F.

- Enlarged main undercarriage sponsons with inlets and exhausts for an undisclosed system (likely laser and systems cooling).

- Removal of the tail gunner station.

Directed energy weapons including the anti-aircraft laser systems such as the recently developed laser close-in weapon system are used in modern warfare. High Energy Liquid Laser Area Defense System (HELLADS) is a counter-RAM aircraft mounted laser under development by General Atomics.

\subsubsection{Laser guided weapons}

A Laser Guided Weapon (LGW) employs a photo-electric sensor designed to recognise the wavelength and the pulse coding of the parent LTD system, consequently the LGW can track the target's relative position and "home" toward it by actuating its aerodynamic surfaces. The effectiveness of LGW systems is substantially dependent upon the pointing accuracy of the laser. In many instances, a slightly modified LRF (pulse coding) is successfully employed as a target designator, and it has the added advantage of simultaneously providing slant range to the target. The LGW (missile or bomb) is designed to track the signal reflected from the target only, and not to follow the beam emitted by the designator (as with laser beam riders). A number of LGW are being increasingly developed by many nations [110-115]. Laser-guided missiles were first developed in 1962 and the first laser-guided bomb named as BOLT-117 was developed by the Air Force in 1967. The BOLT-117 worked using two aircraft. One aircraft was used to keep a laser illuminating the intended target, while the other's job was to drop the missile by following the reflected laser bean and directing the missile by sending signals to its control fins. Two different LGW guidance strategies are currently adopted:

- Bang-bang guidance, in which the LGW only senses a position error, and the control fins are driven to the limit of their travel (generally by high-pressure gas), regardless of the magnitude of the error (i.e., the control fins are either at the trail position or full deflection during guidance);

- Proportional guidance, in which the LGW seeker continuously tracks the maximum of the reflected laser energy and the LGW computer directs towards the target by actuating the weapon aerodynamic surfaces, giving commands proportional to the measured offset.

Dive, level and loft types of attacks are all possible with Laser Guided Bombs (LGB) and a variety of profiles are available with airborne Laser Guided Missiles (LGM) [116]. In general, two 
This is the author pre-publication version. This paper does not include the changes arising from the revision, formatting and publishing process. The final paper that should be used for referencing is:

R. Sabatini, M. A. Richardson, A. Gardi, S. Ramasamy, "Airborne laser sensors and integrated systems", Progress in Aerospace Sciences (2015), http: //dx.doi.org/10.1016/j.paerosci.2015.07.002i.

main categories of attacks with LTD/LGW can be distinguished as depicted in Fig. 11:

- Self-designation Attacks, in which the aircraft acts as illuminator for the own carried LGW and laser illumination is automatically controlled by the LTD, manually controlled by the Weapon System Operator (WSO), or by the aircraft computers (e.g., using a pre-planned counter to be chosen between various mutually exclusive possibilities).

- Co-operative Designation Attacks, in which a ground Forward Air Controller (FAC) (or an aircraft) performs illumination with an LTD for the LGW carried by an (other) aircraft. Automatic steering functions are often implemented in co-operative profiles. In these cases, aircraft are constrained to pass tangent to the target lethal range according to pre-planned steering laws.

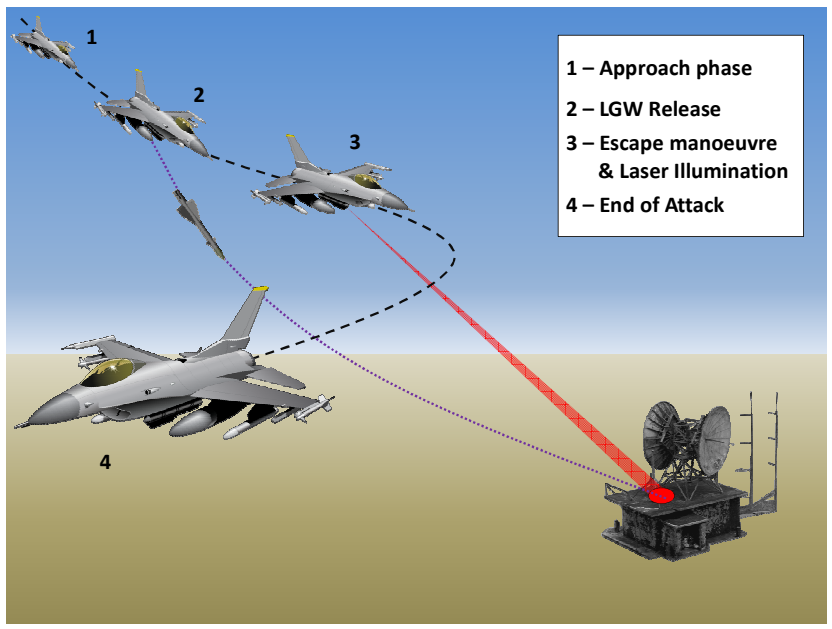

(a) Self-designation attack. Adapted from [12].

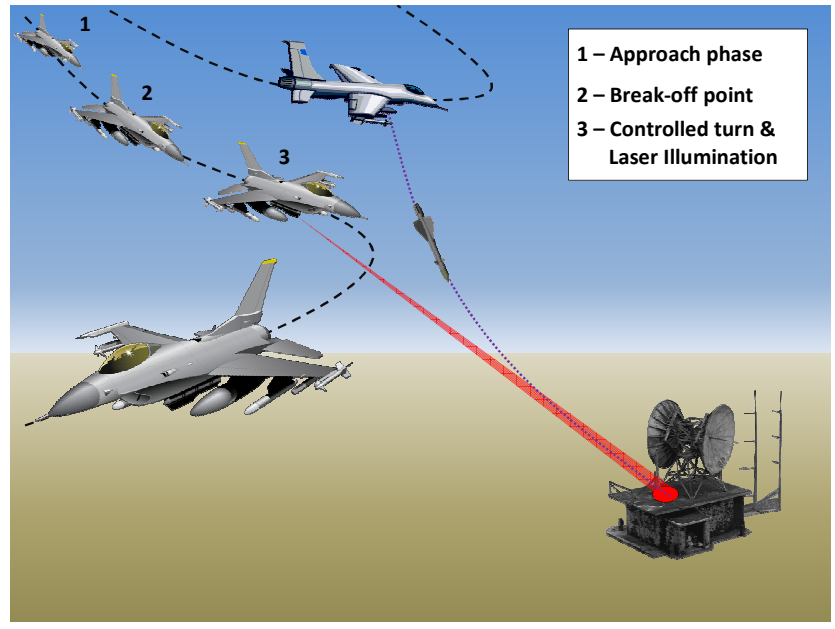

(b) Co-operative attack. Adapted from [12].

Fig. 11. $L T D / L G W$ mission profiles.

The sequence of operations involving the ballistic and homing phases of the LTD/LGW is depicted in Fig. 12. The precise work required by pilots sparked the development of other forms of guided missiles that do not require the pilot's guidance. Additionally, the weather limitations mentioned previously spawned a new breed of missiles that allow for accurate deployment in adverse weather conditions. Such missiles are guided using Global Navigation Satellite Satellite (GNSS) technology. Developed by NASA in 2000, C-band and X-band Interferometric Synthetic Aperture Radars (ISFARs) are used to collect the topographic data required to employ this technology. NASA used these ISFARs to create the most complete and high resolution topography of the Earth available today within ten days, with guided weapons being its primary application. These missiles have a longer range than typical laser-guided missiles [12]. The PGS comprises a Laser Seeker Unit (LSU) which detects reflected laser energy (coded sequences of laser pulses) from the designated target and produces guidance commands to the Pneumatic Actuation System (PAS), according to the target position. The PAS guides the LIZARD by controlling the guidance fins to home on the target. The FTA is used to stabilize the LIZARD after launching and to provide the lift required for manoeuvrability. The system also includes provisions for a GPS add-on kit (to enhance guidance accuracy).

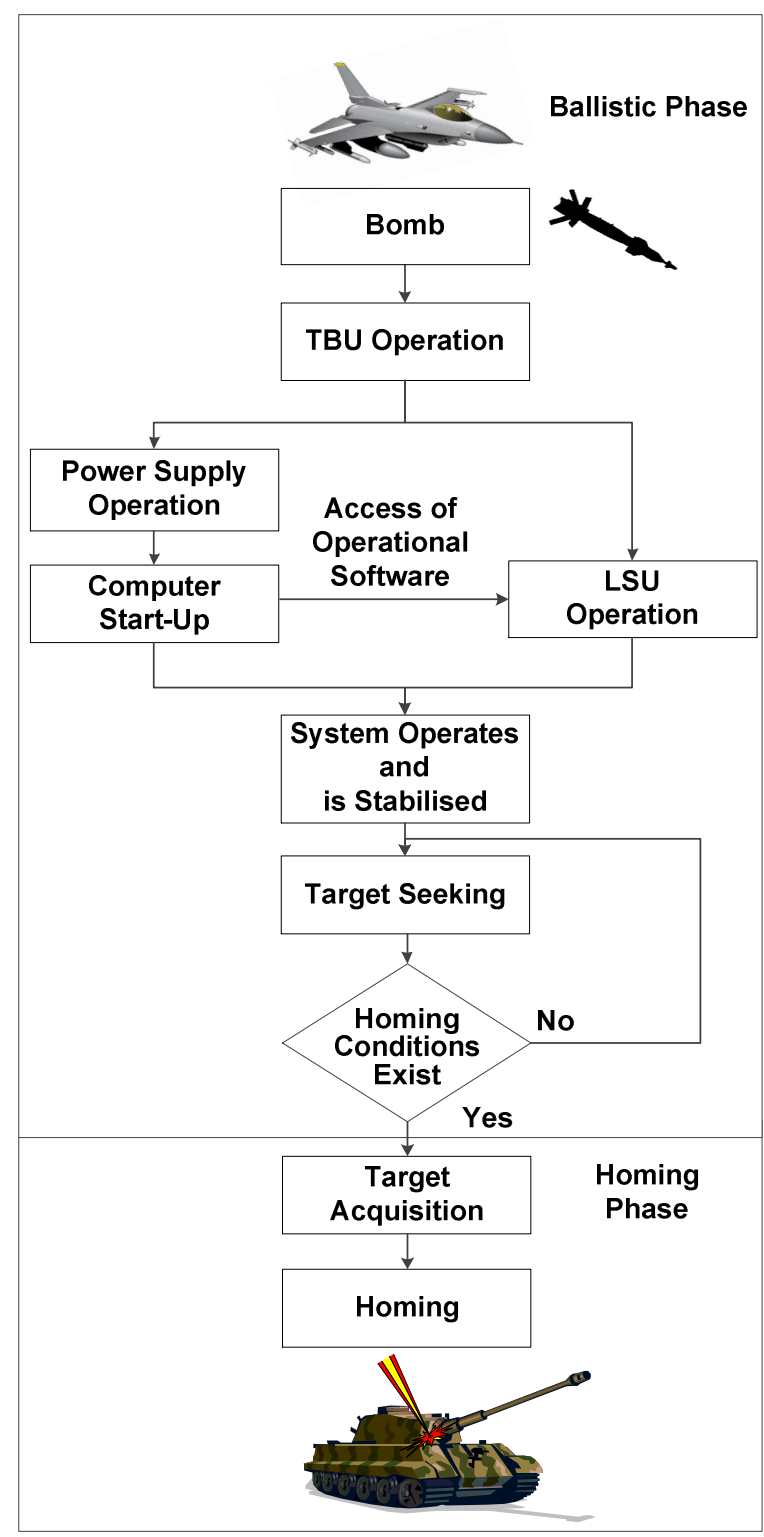

Fig. 12. LTD/LGW sequence of operations. Adapted from [12]. 
This is the author pre-publication version. This paper does not include the changes arising from the revision, formatting and publishing process. The final paper that should be used for referencing is:

R. Sabatini, M. A. Richardson, A. Gardi, S. Ramasamy, "Airborne laser sensors and integrated systems", Progress in Aerospace Sciences (2015), http: //dx.doi.org/10.1016/j.paerosci.2015.07.002i.

\subsubsection{Laser target designators}

Laser Target Designators (LTD) and Laser Guided Weapons (LGW) were developed in order to satisfy the military requirement for weapon systems (i.e., bombs and missiles) capable of pinpoint accuracy, especially when the target is relatively small and well-defended [12,117-120]. A LTD is an accurate pointing system which provides the pulsed laser source and the precision optics and stabilisation required to accurately shine a laser beam on a target. Prior to the development of this technology, there have only been two alternatives to deal with this kind of situation: either get close enough to the target to make certain of a hit or use some kind of blanket bombing over a fairly large area. Closing in to the target may be extremely dangerous and, if it is well defended, could lead to a high casualty rate. On the other hand, blanket bombing may not be effective in destroying the target or may require excessive amounts of ammunition. Furthermore, a concern particularly important in current conflict scenarios is the reduction of collateral damage. This has forced the military into the development of 'smart munitions' which easily pinpoint specific targets. The LTD is an essential element for the operation of these sophisticated weapon systems. For operation of LGW's or 'smart munitions', a coded laser beam from the LTD is directed at the target. The reflected pulses from the target are scattered in many directions. They are detected by the LGW (bomb or missile) target seeker, which is a sensor on the head of the LGW responding to the same code as in the beam. The missile/bomb, which normally is fired from a distant place (e.g., an aircraft), will thus home in on the target and destroy it. From the description given, it appears evident that, with simple design modifications (e.g., specific laser coding), a LRF can serve admirably as a target designator and it has the added advantage of simultaneously providing slant range to the target. In most of the cases, the large availability of laser guided bombs restraints the designators to work at $1.064 \mu \mathrm{m}$, which corresponds to the Nd:YAG laser wavelength.

\subsection{RPAS specific applications}

Mobile Laser Scanning (MLS) is not very efficient for a number of airborne laser based applications including forest foliage scanning. Furthermore, the scanning angles of MLS and airborne LIDAR systems are not consistent. Therefore, an efficient, accurate, low- cost, and flexible surveying scheme with the same scanning view is required to overcome these issues. RPAS as alternative platforms for laser scanning provide a good choice to overcome these challenges. RPAS based LIDAR systems are increasingly developed for a variety of applications. A number of design configurations involving RPAS based laser scanning have been identified [122]. RPAS borne integration systems with a laser scanner, Charge-Coupled Device (CCD) cameras, inertial measurement unit (IMU), and GPS demonstrated the capability to perform airborne 3D mapping [123]. A remotely controlled helicopter installed with GPS and a laser was used for topographic surveys [124]. A miniature laser range finder/altimeter was developed for autonomous navigation and landing in small RPAS [125], and the mini-RPAS optical images were also combined with laser scanning performed on the conventional platforms [126]. One of the key advantages in adopting RPAS based LIDAR applications is that delineation of individual trees in LIDAR point clouds for retrieving tree height and other biophysical, and even biochemical, parameters can be achieved [127]. Data processing involving coordinate triple, pulse intensity, and multiechoes (per pulse) are explored to validate the applicability of mini-RPAS for fine-scale mapping to determine tree height, pole detection, digital terrain model, etc. [128]. Over the past several years there have been a few successful data collections with LIDAR scanners mounted on RPAS platforms [129-131]. All of these systems have taken advantage of lightweight IMU systems and laser scanners in order to limit the overall remote sensing payload. The light payload however comes with a sacrifice to the expected accuracy of the acquired point cloud. Additionally, the advantage of adopting low-cost Micro Electro-Mechanical Sensors (MEMS) are typically considered, which have comparably high noise values and drift rates that significantly degrade the expected accuracy of their attitude solution. A number of Terrain Referenced Navigation (TRN) techniques are employed to constrain the error in an integrated navigation system. A TRN based on a single ALS system, an inertial sensor and an on-board terrain database was used to perform multiple precision approaches achieving meter- level accuracies [129]. Remote sensing employing RPAS is increasingly used to survey forests and urban areas. Mobile surveys in GNSS-denied below-canopy forest environments are also carried out using RPAS. An example of such application is a battery- powered RPAS equipped with a LIDAR, which lacks GNSS or any other localisation device. A number of applications have been developed specifically for RPAS based on airborne lasers including below-canopy forest surveys, surveying, land information map- ping, forest inventory, pollution monitoring and non-cooperative obstacle avoidance [132-136].

\subsection{Laser communication systems and data links}

Modern battlefield strategy is based on determining where the enemy's (or potential enemy's) assets are located and what is their operational capability. This vital information is constantly being gathered and updated by various ground, space and airborne sensors. The requirement to send ever increasing amounts of tactical information between sensor aircraft and information processing facilities has highlighted the limits of present airborne data links, even when data compression techniques are used. Communication using laser at short wavelengths holds several advantages over other communication systems available in the Radio-Frequency (RF) spectrum including high signal strength (less prone to loss), lower power consumption, more compact equipment and high imperviousness to jamming. Therefore, utilization of optical data links is being considered as a promising solution. Laser-based communication has higher bandwidth and speed, and requires no compression. Laser communication system design involves careful considerations for health and safety by operating in the eye-safe region. Hybrid communication systems are also deployed by including laser wireless transceiver operating by switching to RF wireless transceiver. The hybrid systems in- creases the data rate in addition to providing an all-weather and all-time operational capability. The laser communication system can be modelled using a transmitter, channel and receiver. The transmitter typically includes a light source, modulator and the associated optics. The wireless channel is modelled by including associated effects such as attenuation, spreading and widening of the signal. The receiver typically includes a detector, filter, amplifier and the associated optics. Successful tests in space, over land and under water were conducted by major research organisations and attested the viability of laser-light data transmission systems. Optical communication, with its ability to port data at gigabit speeds, is a boon for any application that relies on networkcentric operations. A combination of incoherent transmission technology and point-to-point and point-to multi-point topologies enables networked communication among multiple transmitters and receiving terminals, and increases availability. The 
This is the author pre-publication version. This paper does not include the changes arising from the revision, formatting and publishing process. The final paper that should be used for referencing is:

R. Sabatini, M. A. Richardson, A. Gardi, S. Ramasamy, "Airborne laser sensors and integrated systems", Progress in Aerospace Sciences (2015), http: //dx.doi.org/10.1016/j.paerosci.2015.07.002i.

feasibility of laser airborne data links was demonstrated in the mid-80's by the U.S. Air Force Research Laboratory Laser Airborne Communications Experiment (HAVE LACE) Program $[137,138]$. This program developed and tested two laser communications terminals that operated at $19.2 \mathrm{kbps}$. The terminals were tested using two KC-135 aircraft that nominally flew at 20,000 to 25,000 feet (ft) altitudes with separation distances up to $160 \mathrm{~km}$. The most significant result of the HAVE LACE flights was the difficulty of initial signal acquisition between the two moving platforms, since it had to be per- formed manually. However, once signal acquisition was accomplished, tracking proved to be robust and communications performance was consistently measured at 10-6 Bit Error Rate (BER) or better. Since the HAVE LACE program, laser terminal development and data rates have improved dramatically. Therefore, various re- search programs have been undertaken in order to fully exploit the potentials of this technology, mainly for space borne and airborne applications. Another U.S. Air force program was to develop a wideband laser data link operating at 810 and $852 \mathrm{~nm}$. In September 995, the program successfully ground demonstrated a 1.1 Gbps full duplex data link over a distance of $150 \mathrm{~km}$ (Hawaii Islands) at an altitude of 10,000 ft. Successively the system used in the ground demonstration was redesigned and installed in two jet aircraft for flight demonstration at distances up to $500 \mathrm{~km}$. The demonstration flights, performed in September 1998, were successful and proved the ability of the system to communicate in the upper atmosphere to $500 \mathrm{~km}$ with a BER of $10^{-6}$. Efforts were also made to develop two laser communication transceivers to be externally mounted on two T-39 test aircraft and used to demonstrate communication between the aircraft at data rates of $1 \mathrm{Gbps}$ at ranges between 50 and $500 \mathrm{~km}$ and altitudes up to the $40,000 \mathrm{ft}$. Since airborne laser-communication systems are subjected to specific size, complexity, power, and weight requirements, the reduction of the received signal variability by implementing optimized multiple-transmitter systems to average out the effects of turbulence by including anisoplanatic conditions. Semiconductor-laser Inter-Satellite Link Experiment (SILEX) by the French National Space Study Center (NES), Laser Communication Link (LCT) for inter satellite links by DLR and Kirari Optical Communication Demonstration Experiments (KODEN) experiments by Japan Aerospace Exploration Agency (JAXA) [149].

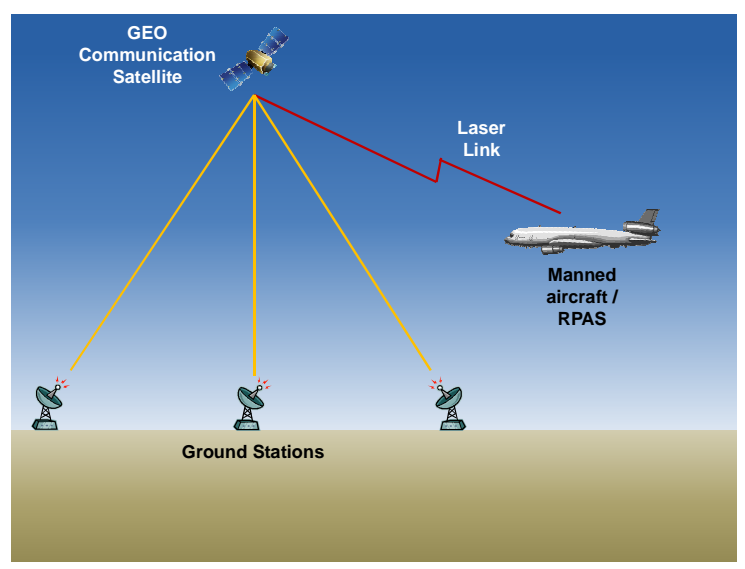

Fig. 13. Geostationary satellite based airborne laser link.

The Lunar Laser Communication Demonstration (LLCD) on the NASA's Lunar Atmosphere and Dust Environment Explorer (LADEE) spacecraft achieved higher data speeds from the Moon. NASA's LLCD recently used a pulsed laser beam to transmit data from the Moon to Earth at a download rate of $622 \mathrm{Mbps}$, which is higher than six times faster than the best radio system ever flown to the Moon with less weight and power. The reductions in mass and power consumption obtained by the adoption of laser communication systems allows for increasing the fuel and payload capacities. An illustration of geostationary satellite based airborne laser link is represented in Fig. 13.

\section{Airborne Lasers Performance Analysis}

This section presents the fundamental relationships to estimate the performance of airborne laser systems. These are required for design purposes as well as for experimental activities with airborne laser systems, including both developmental and operational test and evaluation in the laboratory and in flight.

\subsection{Laser range equation}

The generic form of the microwave radar range equation also applies to laser systems [150]:

$$
P_{R}=\frac{P_{T} G_{T}}{4 \pi R^{2}} \times \frac{\sigma}{4 \pi R^{2}} \times \frac{\pi D^{2}}{4} \times \tau_{a t m} \tau_{s y s}
$$

where $P_{R}$ is the received signal power $(\mathrm{W}), P_{T}$ is the transmitter power $(\mathrm{W}), G_{T}$ is the transmitter antenna gain, $\alpha$ is the transmitter beamwidth, $\sigma$ is the effective target cross section $\left(\mathrm{m}^{2}\right), K_{a}$ is the aperture illumination constant, $R$ is the system range to target (m), $\lambda$ is the wavelength $(\mathrm{m}), D$ is the aperture diameter $(\mathrm{m}), \tau_{a t m}$ is the atmospheric transmission factor and $\tau_{s y s}$ is the system transmission factor. With laser systems, the transmitter antenna gain is substituted by the aperture gain, expressed by the ratio of the steradian solid angle of the transmitter beamwidth $\alpha^{2}$ to that of the solid angle of a sphere, which is equal to the relation, [150]:

$$
G_{T}=\frac{4 \pi}{\alpha^{2}}
$$

For laser beam widths on the order of $1 \mathrm{mrad}$, the typical aperture gain at laser wavelengths is about $70 \mathrm{~dB}$. In the far field, the transmitter beam width can also be expressed as:

$$
\alpha=K_{a} \frac{\lambda}{D}
$$

Substituting the above expressions for transmitter aperture gain (4) and beamwidth (5), eq. (3) becomes:

$$
P_{R}=\frac{P_{T} \sigma D^{4} \tau_{a t m} \tau_{s y s}}{16 R^{4} \lambda^{2} K_{a}^{2}}
$$

Eq. (4), obtained from the standard radar range equation, applies only in the far field of the aperture. At typical microwave bands of $\lambda=1$ to $10^{-3} \mathrm{~m}$, the far-field distances are quite short, as shown in Fig. 14. The far-field (Fraunhofer) region of an aperture is typically concerned with the distance $2 D^{2} / \lambda$ to infinity; in this vicinity, the generalised range equation applies. In certain cases, the far field distance occurs within the feed horn assembly of a microwave antenna. As illustrated by the figure, at $\lambda=1.064 \mu \mathrm{m}$ (Nd:YAG laser), a $10 \mathrm{~cm}$ aperture has a far-field distance of approximately $20 \mathrm{~km}$. As a result, it is not unusual to operate in the near-field of the optical systems; thus modifications to the range equation to account for near-field operation are required. This near-field effect modifies the beam width such that:

$$
\alpha=\sqrt{\left(\frac{K_{a} D}{R}\right)^{2}+\left(\frac{K_{a} \lambda}{D}\right)^{2}}
$$


This is the author pre-publication version. This paper does not include the changes arising from the revision, formatting and publishing process. The final paper that should be used for referencing is:

R. Sabatini, M. A. Richardson, A. Gardi, S. Ramasamy, "Airborne laser sensors and integrated systems", Progress in Aerospace Sciences (2015), http: //dx.doi.org/10.1016/j.paerosci.2015.07.002i.

\subsection{Comparison of incoherent and coherent detection techniques}

Fig. 15 shows the illustrations relative to incoherent detection and coherent detection receivers respectively [151]. Incoherent detection receivers at optical wavelengths are similar to video radiometers receivers (i.e., envelope detectors at microwave wavelengths). However, optical receivers have an additional term besides the signal term $\left(P_{S I G}\right)$, the optical background power $\left(P_{B K}\right)$ which is due to undesired signals such as sunlight, cloud reflections, flares, etc. The received optical power, after suitable filtering, is applied to the optical detector. Square law detection then occurs, producing a video bandwidth electrical signal. The coherent detection receiver is similar to the incoherent; however, a portion of the laser signal $\left(f_{o}\right)$, is coupled to the optical detector via beam splitters. As a result, the optical detector has the local oscillator power $\left(P_{L O}\right)$ in addition to the received signal power $\left(P_{S I G}\right)$, and the competing background terms $\left(P_{B K}\right)$.

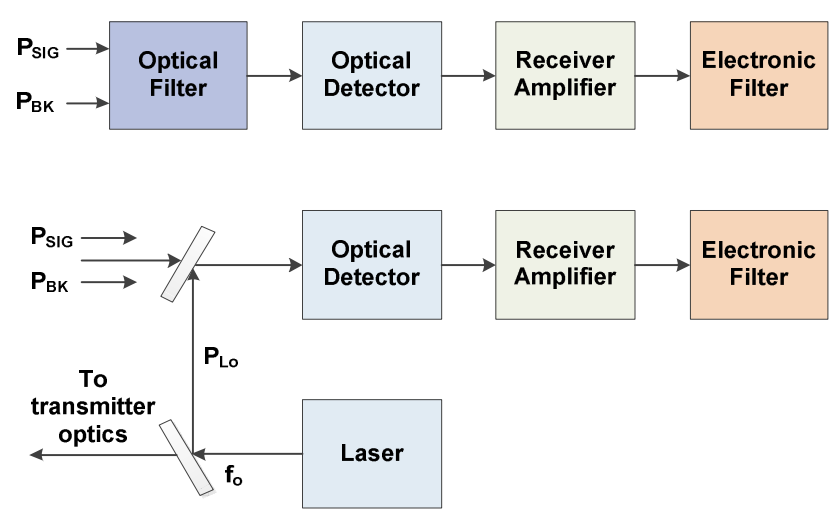

Fig. 14. Laser receiver systems.

In general, the signal-to-noise ratio (SNR) of a LIDAR system can be expressed in the form [12]:

$$
S N R=\frac{i_{S I G}{ }^{2}}{i_{S N}{ }^{2}+i_{T H}{ }^{2}+i_{B K}{ }^{2}+i_{D K}{ }^{2}+i_{L O}{ }^{2}}
$$

where $i_{S I G}{ }^{2}$ is the mean square signal current, $i_{S N}{ }^{2}$ is the mean square shot noise current, $i_{T H}{ }^{2}$ is the mean square thermal noise current, $i_{B K}{ }^{2}$ is the mean square background noise current, $i_{D K}{ }^{2}$ is the mean square dark noise current and $i_{L O}{ }^{2}$ is the mean square local oscillator noise current. Considering the various receiver noise and signal terms, two forms of the SNR equation can be obtained, for incoherent and coherent detection systems. The SNR for the incoherent system has the received signal power squared in its numerator, and has a summation of noise terms associated with the return signal, the background signal, the dark current, and the thermal noise of the receiver in the denominator. The returned signal power and the background power are included as noise sources in the detection process because of the random photon arrival rate (Poisson noise). In the coherent detection system, the local oscillator power is an additional source of noise (compared to the incoherent system), and the numerator is related to the product of the received signal power and the local oscillator power. The local oscillator power is very important in the detection process; here, it may be increased so that it overwhelms all of the other noise sources. As a result, the local oscillator power in the denominator cancels out the local oscillator power in the numerator; the SNR is directly proportional to the received signal power, rather than to the received signal power squared (as with the incoherent system). Additionally, because the local oscillator power becomes the predominant noise source, the coherent detection system typically is background immune, since only signals that are phase coherent with the local oscillator are efficiently detected. For a typical SNR requirement of 100 (20 $\mathrm{dB}$ ), the coherent system is seen to have a $30-\mathrm{dB}$ increased sensitivity over that of an incoherent system. The transmitter power for a range of SNR values for both coherent and incoherent detection is depicted in Fig. 15 [30].

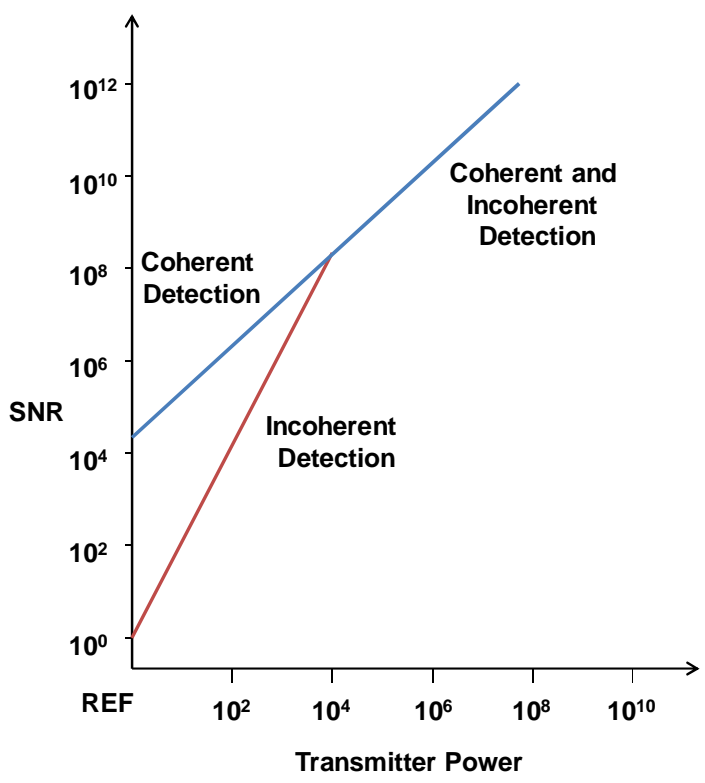

Fig. 15. SNR versus transmitter power for coherent and incoherent detection.

\subsection{Laser beam propagation in the atmosphere}

In general, a laser beam is attenuated as it propagates through the atmosphere, mainly due to absorption and scattering phenomena. Additionally, the laser beam is often broadened, defocused, and may even be deflected from its initial propagation direction. On one hand when the output power is low, the effects are linear in behaviour (absorption, scattering, and atmospheric turbulence are examples of linear effects). On the other hand, when the power is sufficiently high, new effects are observed that are characterised by non-linear relationships (e.g., thermal blooming, kinetic cooling, bleaching and atmospheric breakdown). In both cases, the atmospheric effects can be significant and severely limit the usefulness of the beam.

\subsubsection{Atmospheric transmittance for airborne lasers}

Attenuation of laser radiation in a generic medium is described by the Beer's law:

$$
\tau=e^{-\int_{0}^{z} \gamma(z) d z}
$$

where $\tau$ is the transmittance, $\gamma$ is the attenuation coefficient, and $z$ is the length of the transmission path. The attenuation coefficient is determined by four individual processes: molecular absorption, molecular scattering, aerosol absorption, and aerosol scattering. The atmospheric attenuation coefficient is:

$$
\gamma=\alpha_{m}+\beta_{m}+\alpha_{a}+\beta_{a}
$$

where $\alpha$ is the absorption coefficient, $\beta$ is the scattering coefficient, and the subscripts $m$ and $a$ designate the molecular and aerosol processes, respectively. Each coefficient in eq. (10) 
This is the author pre-publication version. This paper does not include the changes arising from the revision, formatting and publishing process. The final paper that should be used for referencing is:

R. Sabatini, M. A. Richardson, A. Gardi, S. Ramasamy, "Airborne laser sensors and integrated systems", Progress in Aerospace Sciences (2015), http: //dx.doi.org/10.1016/j.paerosci.2015.07.002i.

depends on the wavelength of the laser radiation. It is convenient at times to discuss absorption and scattering in terms of the absorption and scattering cross sections ( $\sigma_{a}$ and $\sigma_{s}$, respectively) of the individual particles that are involved. Thus, we have:

$$
\alpha=\sigma_{a} N_{a}
$$

and

$$
\beta=\sigma_{s} N_{s}
$$

where $N_{a}$ and $N_{s}$ are the concentrations of the absorbers and scatterers, respectively. In the absence of precipitation, the atmosphere contains finely dispersed solid and liquid particles (of ice, dust, aromatic and organic material) that vary in size from a cluster of a few molecules to particles of about $20 \mu \mathrm{m}$ in radius. Particles larger than this remain airborne for a short time and are only found close to their sources. Such a colloidal system, in which a gas (in this case, air) is the continuous medium and particles of solid or liquid are dispersed, is known as an aerosol. Aerosol attenuation coefficients depend considerably on the dimensions, chemical composition, and concentration of aerosol particles. These particles are generally assumed to be homogeneous spheres that are characterized by two parameters: the radius and the index of refraction. In general, the index of refraction is complex. Therefore, we can write:

$$
\check{n}=n-i k=n\left(1-i \frac{k}{n}\right)=n(1-i k)
$$

where $n$ and $k$ are the real and imaginary parts and $K=\frac{k}{n}$ is known as the extinction coefficient. In general, both $n$ and $k$ are functions of the frequency of the incident radiation. The imaginary part (which arises from a finite conductivity of the particle) is a measure of the absorption. In fact, $k$ is referred to as the absorption constant. It is related to the absorption coefficient $\alpha$ by:

$$
\alpha=\frac{4 \pi f k}{c}
$$

where $c$ is the speed of light in a vacuum and $\mathrm{f}$ is the frequency of the incident radiation. The atmospheric spectral transmittance $\tau(\%)$ measured over a $1820-\mathrm{m}$ horizontal path at sea level is shown in Fig. 16 [30].

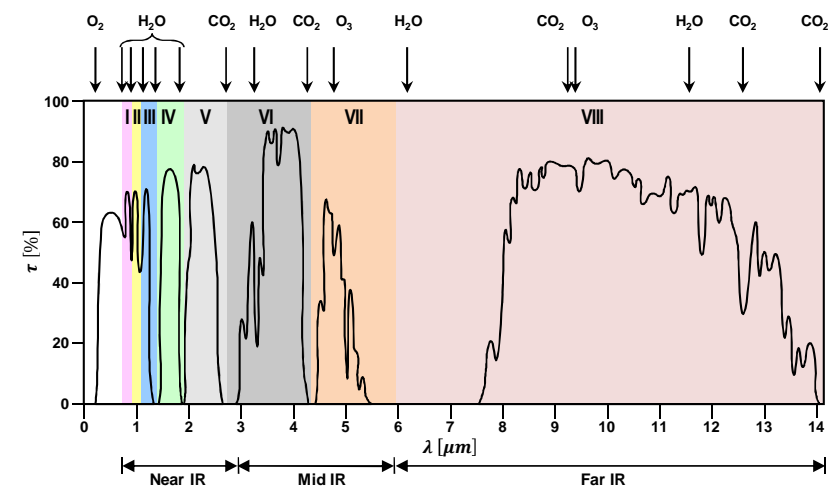

Fig. 16. Sea-level transmittance over a $1820 \mathrm{~m}$ horizontal path

For the wavelength range of greater interest in laser beam propagation (the visible region to about $15 \mu \mathrm{m}$ ) the principal atmospheric absorbers are the molecules of water, carbon dioxide, and ozone. Attenuation occurs because these molecules selectively absorb radiation by changing vibrational and rotational energy states. The two gases present in greatest abundance in the earth's atmosphere, nitrogen $\left(\mathrm{N}_{2}\right)$ and oxygen $\left(\mathrm{O}_{2}\right)$, are homonuclear, which means that they possess no electric dipole moment and therefore do not exhibit molecular absorption bands. The molecule responsible for each absorption band is shown in the upper part of Fig. 17. It is evident that $\mathrm{H}_{2} \mathrm{O}$ and $\mathrm{CO}_{2}$ are by far the most important absorbing molecules. Other absorbing molecules found in the atmosphere are methane $\left(\mathrm{CH}_{4}\right)$, with an average concentration of around $1.5 \times 10^{-4}$ percent; nitrous oxide $\left(\mathrm{N}_{2} \mathrm{O}\right)$, with an average concentration of around $3.5 \times 10^{-5}$ percent; carbon monoxide $(\mathrm{CO})$ with a typical concentration of $2 \times 10^{-5}$ percent; and ozone $\left(\mathrm{O}_{3}\right)$, with a concentration as large as $10^{-3}$ percent at an altitude of around $30 \mathrm{~km}$. The wavelength intervals where the transmittance is relatively high are called "atmospheric windows". There are a total of eight such windows within the wavelength range extending from 0.72 to $15.0 \mu \mathrm{m}$. The window boundaries are listed in Table 4.

\section{Table 4}

Wavelength regions of atmospheric windows.

\begin{tabular}{ccc}
\hline Window Number & \multicolumn{2}{c}{ Window Boundaries $(\boldsymbol{\mu m})$} \\
I & 0.72 & 0.94 \\
II & 0.94 & 1.13 \\
III & 1.13 & 1.38 \\
IV & 1.38 & 1.90 \\
V & 1.90 & 2.70 \\
VI & 2.70 & 4.30 \\
VII & 4.30 & 6.00 \\
\hline
\end{tabular}

\subsubsection{Molecular absorption and scattering}

The interactions of laser radiation with the fine structure of atmosphere are not similar to conventional optical systems due to its highly monochromatic nature. The dominating attenuation phenomena include molecular absorption and scattering. The fine structure of the atmosphere has significantly different effects on the atmospheric transmission in both the transmitted and received paths of an active system like a LRF or a LADAR when compared to conventional electro-optic systems. Doppler shift causes higher attenuation if the transmitter or the target is in motion, due to the shift in carrier wavelength on transmission or reflection.

\subsubsection{Molecular line absorption}

Considering molecular absorption only, the fraction of monochromatic radiation transmitted (or the transmittance) is given by:

$$
\tau(\lambda)=e^{-\alpha(\lambda) Z}
$$

were $\alpha(\lambda)$ is the absorption coefficient and $z$ is the path length. Eq. (18) is valid when the absorption coefficient is constant for the entire path length $z$. To describe slant path propagation where $\alpha(\lambda)$ is not a constant over the path length, one uses the more general expression:

$$
\tau(\lambda)=e^{-\int_{0}^{l} \sigma_{a}(\lambda) N d r}
$$

where $\sigma_{a}(\lambda)$ is the absorption cross section, $N$ is the number density of absorbing molecules, and the integration extends over the propagation range of length $l$. 
This is the author pre-publication version. This paper does not include the changes arising from the revision, formatting and publishing process. The final paper that should be used for referencing is:

R. Sabatini, M. A. Richardson, A. Gardi, S. Ramasamy, "Airborne laser sensors and integrated systems", Progress in Aerospace Sciences (2015), http: //dx.doi.org/10.1016/j.paerosci.2015.07.002i.

\subsubsection{Continuum absorption}

In addition to molecular absorption by discrete absorption lines, there exists a slowly varying component of molecular absorption in the atmosphere caused mainly by molecular clusters. This absorption plays a fundamental role particularly in "window" regions where absorption by discrete lines is small. It is difficult to separate the cluster molecular absorption from absorption in the distant wings of strong discrete absorption lines. For practical reasons, far wing absorption and cluster absorption are combined and called "continuum" absorption. In regions of more substantial line absorption, the problem reduces to that of deciding how far into the wings of each line to assume individual line contributions and how much of the experimentally observed absorption to model as "continuum". Various models have been developed for continuum absorption contributions in the various atmospheric windows. A comprehensive collection of these models is presented in [152]. However, it should be underlined that, because of the considerable lack of experimental data, related to the physical complexity of continuum absorption processes, there is still some uncertainty in the continuum contributions.

\subsubsection{Atmospheric scattering}

In this process there is no loss of energy but only a directional redistribution which may lead to a significant reduction in beam intensity for large path lengths. For example, on a clear day scattering reduces transmission in the visible spectrum to $20 \%$ for a $16 \mathrm{~km}$ path. The physical size of the scatterer determines the type of scattering. Thus, air molecules that are typically several angstrom units in diameter (larger than electron but smaller than 2) lead to Rayleigh scattering, whereas the aerosols (comparable in size to $\lambda$ ) scatter light in accordance with the Mie theory. Furthermore, when the scatterers are relatively large (much larger than $\lambda$ ), such as the water droplets found in fog, clouds, rain, or snow, the scattering process is more properly described by diffraction theory.

\section{Rayleigh scattering}

Rayleigh scattering is due to the displacement of bound electrons by the incident field. The process is schematically illustrated in Fig. 17. The incident harmonic field induces a dipole in the molecule whose polarisation determines the displacement.

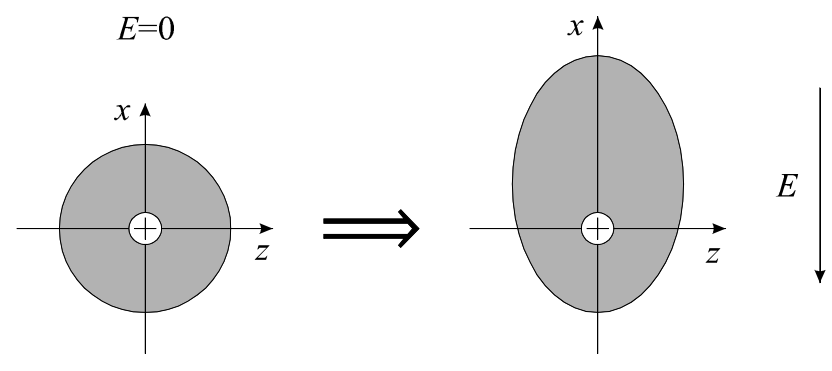

Fig. 17. Induced dipole in presence of applied harmonic field E.

The induced dipole oscillates at the same frequency as the incident and emits electromagnetic radiation whose spatial distribution is shown in Fig. 18. This reradiated energy constitutes the scattered light.

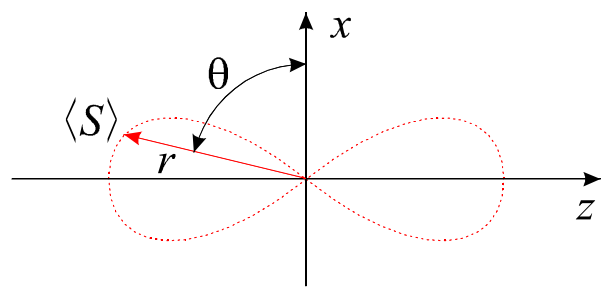

Fig. 18. Spatial distribution of radiated energy flux (S).

Using the laws of classical electrodynamics, it can be shown that the scattering cross-section of a single dipole radiator is given by [152]:

$$
\sigma_{s}=\frac{\left(\frac{e^{2}}{m}\right)^{2} \omega^{4}}{6 \varepsilon_{0}^{2} \pi c^{2}\left[\left(\omega_{0}^{2}-\omega^{2}\right)^{2}+(\Gamma \omega)^{2}\right]}
$$

where $\omega_{0}$ is the natural frequency, $e$ is the electron charge, $\omega$ is the applied frequency, and $\Gamma$ is the damping coefficient. For the special case where the applied frequency $\omega$ is much lower than the natural frequency $\omega_{0}$ and where the damping coefficient $\Gamma$ is small, eq. (18) reduces to:

$$
\sigma_{s}=\frac{\left(\frac{e^{2}}{m}\right)^{2}}{6 \varepsilon_{0}^{2} \pi c^{4}}\left(\frac{\omega}{\omega_{0}}\right)^{4}, \quad \text { for } \omega<\omega_{0} \text { and small } \Gamma
$$

\section{Mie scattering}

Reyleigh scattering equations are based on the assumption that the wavelength of the laser beam was considerably longer than the radius of the molecule (i.e., $\lambda>>$ a). This assumption permits to ignore the spatial variation of the electric field over the molecular charge distribution. Because of this assumption, the results obtained in the previous paragraph are not applicable when the scattering particles have radii that are comparable to the wavelength. Gustav Mie, a German meteorologist, was the first to study the scattering of electromagnetic waves by small dielectric spheres. Mie scattering in the atmosphere is caused by the ever-present aerosol particles and by small water droplets. Mie's scattering theory takes into account the size, shape, dielectric constant, and absorptivity of the particle. One important result of this theory is that particles with the same product of the particle radius, a and propagation constant, $\mathrm{k}$ have the same scattering properties. The product ka along with the refractive index difference $\Delta \mathrm{n}$ between the aerosol particle and the air determine all scattering characteristics. To calculate the Mie attenuation coefficient we refer to the experiment illustrated in Fig. 19. A monochromatic beam of light of cross-sectional area (A) and intensity (I) is incident on a cell of volume $\mathrm{V}=\mathrm{Adz}$ containing an aerosol with identical spherical particles of radius a. The total cross section of the particles is $\sigma_{T}=N A d z \pi a^{2}$ if no one particle shields another particle. Because of absorption in addition to scattering, the effective attenuation cross section per particle is larger by the factor $\mathrm{K}$, where $\mathrm{K}$ is the so-called attenuation factor and $\mathrm{K} \pi \mathrm{a}^{2}$ the attenuation cross section. If no absorption occurs, then the attenuation cross section is equal to the scattering cross section. 
This is the author pre-publication version. This paper does not include the changes arising from the revision, formatting and publishing process. The final paper that should be used for referencing is:

R. Sabatini, M. A. Richardson, A. Gardi, S. Ramasamy, "Airborne laser sensors and integrated systems", Progress in Aerospace Sciences (2015), http: //dx.doi.org/10.1016/j.paerosci.2015.07.002i.

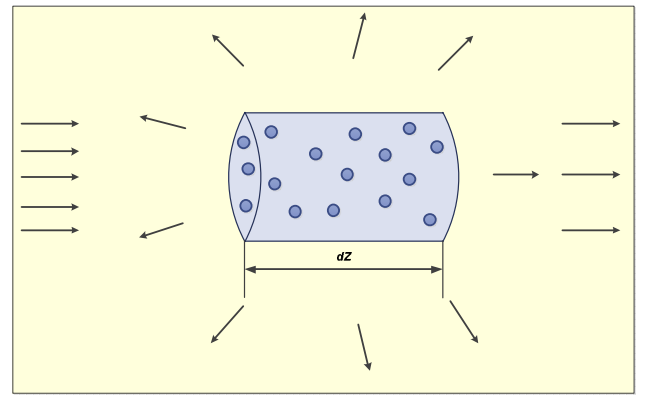

Fig. 19. Experiment to illustrate Mie scattering.

The fractional decrease in intensity of the monochromatic beam of light as it propagates through the cell is then:

$$
-\frac{d I}{I}=\frac{K \pi a^{2} N A d z}{A}=N \sigma(a, \lambda) d z
$$

where $N \sigma(a, \lambda)$ is the Mie attenuation coefficient and $\sigma=$ $K \pi a^{2}$ is the Mie attenuation cross section. Thus, the Mie attenuation coefficient is equal to the product of the number density $N$ of aerosol particles and the extinction cross section. Since the energy removed is partly due to scattering and partly due to absorption by the particle, the attenuation factor $K$ consists of a scattering and absorption term:

$$
K(a, \lambda)=K_{s}+K_{a}
$$

More generally, for particles with a size distribution $N(a)$, where $N(a) d a$ is the number of particles per unit volume within the range $a$ to $a+d a$, the fractional change in the beam intensity is:

$$
-\frac{d I}{I}=d z \int_{0}^{\infty} N(a) \sigma(a, \lambda) d a
$$

Integrating eq. (22) yields:

$$
I=I_{0} e^{-\int \gamma d z}
$$

where:

$$
\gamma=\int_{0}^{\infty} N(a) \sigma(a, \lambda) d a
$$

The value of $K(a, \lambda)$ can be calculated from Mie's scattering theory. The value of $\mathrm{K}(\mathrm{a}, \lambda)$ can be calculated from Mie's scattering theory. A typical result for small water droplets having a refractive index $n=1.33$ is shown in Fig. 20 .
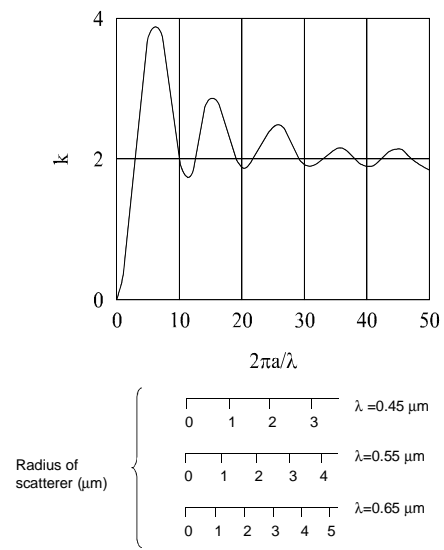

Fig. 20. Plot of the Mie attenuation factor $\mathrm{K}$ for water droplets with $\mathrm{n}=1.33$.

\subsubsection{Nonlinear propagation effects}

The propagation of a laser beam through atmospheric turbulence is a linear phenomenon in that the air is not affected by the beam. Strictly speaking, this is only true for beams of relatively low irradiance. As the beam irradiance is increased, molecular absorption will lead to temperature gradients in the medium that in turn induce density and index-of-refraction changes. The final result is a medium whose optical properties have been altered. This phenomenon is non-linear, in that the beam's irradiance distribution leads to index-of-refraction changes, which in turn alter the beam's irradiance distribution, which alters the refractive index, etc.

\subsubsection{Thermal blooming}

One effect that can be attributed to the beam-induced index-ofrefraction change is a defocusing, or blooming, of the beam. The divergence angle is considerably more than that due to diffraction alone. This thermo-optical effect is conceptually represented in Fig. 21. A high-irradiance CW beam with a Gaussian irradiance profile propagates through stationary air characterised initially (at $t=0$ ) by a constant temperature $T_{0}$, density $\rho_{0}$, and index of refraction $n_{0}$. Upon absorption of laser photons by the air the energy is quickly released as heat, and the temperature of the air within the beam rises. The hot air near the centre of the beam axis expands radially outward (at the speed of sound). The expansion causes a decrease in the mass density, which in turn lowers the index of refraction near the beam's axis. The beam now undergoes a weak, but nevertheless observable, defocusing not unlike that which takes place when a beam is passed through a negative lens.
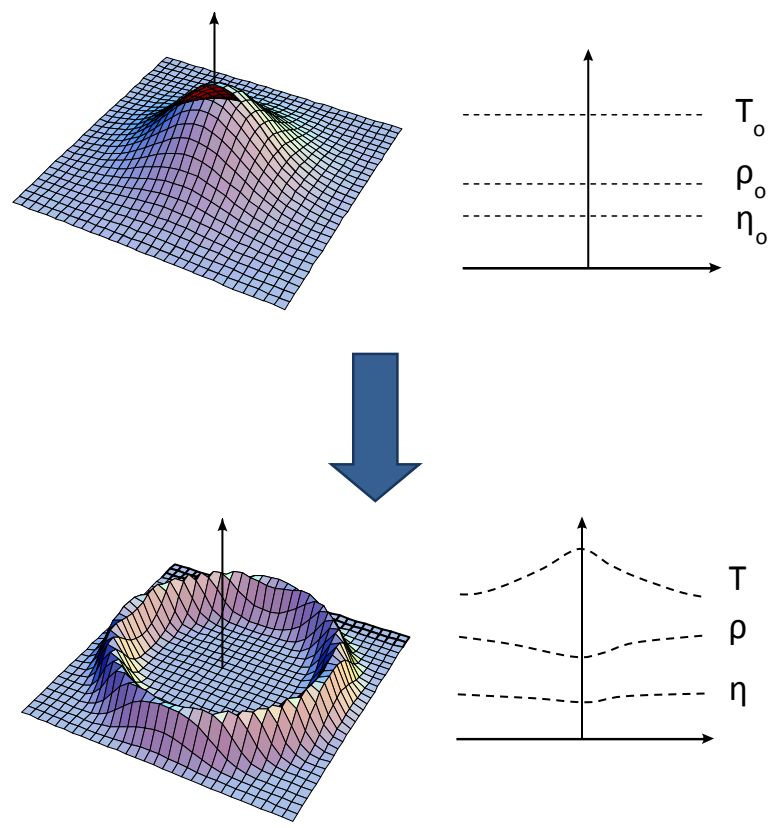

Fig. 21. Irradiance profile of an initially Gaussian $\mathrm{CW}$ beam propagating through stationary air that at $\mathrm{t}=0$ has a constant temperature $\mathrm{T}_{0}$, density $\rho_{0}$, and index of refraction $n_{0}$.

\subsubsection{Kinetic cooling of the atmosphere}

Assuming that the dissipated laser beam energy directly increases temperature of the column within the beam only is inaccurate. For example, the temperature of a parcel of air entering a $\mathrm{CO}_{2}$ laser 
This is the author pre-publication version. This paper does not include the changes arising from the revision, formatting and publishing process. The final paper that should be used for referencing is:

R. Sabatini, M. A. Richardson, A. Gardi, S. Ramasamy, "Airborne laser sensors and integrated systems", Progress in Aerospace Sciences (2015), http: //dx.doi.org/10.1016/j.paerosci.2015.07.002i.

beam may initially drop before it rises beyond the ambient temperature. Such transient cooling, or kinetic cooling, causes the refractive index of the air to rise near the beam axis. This leads to a temporary focusing effect and less than diffraction limited beam spreading.

\subsubsection{Bleaching}

The thermo-optic phenomena for laser beams with pulse durations $t$ that were longer than the hydrodynamic time $w / v_{s}$, where $w$ is the beam spot size and $v$, is the speed of sound are normally considered. By using pulses of time duration $\mathrm{t} \ll w / v_{s}$, the deleterious effects of the thermo-optic phenomena may be completely removed. However, pulses of such short duration (about 1 to $5 \mu \mathrm{sec}$ ) may under certain conditions saturate the absorption mechanism and thereby modify the atmospheric transmittance. The saturation of the absorption mechanism is referred to as bleaching. In general, low irradiance pulses are strongly altered in both pulse shape and pulse energy, while high irradiance pulses are deformed only on the leading edge and the pulse is only weakly attenuated.

\subsubsection{Aerodynamic effects}

Optical aberrations resulting from aerodynamic flow fields can include:

- viscous flow phenomena including laminar and turbulent boundary layers, shear layers, and wake turbulence;

- inviscid or potential flow, arising from the acceleration of (compressible) fluid, generally along the fuselage contours or around protuberances.

For wavelengths ranging from the visible to the mid-IR, flushmounted material windows generally can be employed to shield the optical components from the external aerodynamic flow field. An exception may occur if a high-power laser is to be transmitted through the window; in this case, thermal-induced distortions or potential damage may preclude its use. If such installations are feasible, then the aeromechanical issue is only associated with the aircraft motion. The resulting aero-optical performance is driven by the aerodynamic flow field that exists over the external portion of the aircraft and window itself. These aerodynamic flow fields, if laminar, tend to be relatively benign and are influenced by the mean flow field that exist over the aerodynamic contours. However, if the surface boundary layer over the window is turbulent, as is more likely in operational settings, the resulting unsteady air density variations (resulting in unsteady index of refraction fields) can yield significant optical aberrations [154]. For long-wavelength IR transmission and reception $(\lambda \geq 10 \mu \mathrm{m})$, a material window becomes much less attractive owing to optical transmission losses and window radiative effects. For such applications, cavities that are open to the surrounding aerodynamic flow field may be used. Also in this case, however, the AO flow field can cause a whole range of optical aberrations (e.g., considerable intensity reductions due to scattering, absorption, refraction and reflection by the AO interface, etc.). It is evident that an effective control of these aberrations is a prerequisite to any airborne laser application. Once the basic requirements of an airborne laser system have been defined, some scaling relations may be used to prioritise the undesired $\mathrm{AO}$ effects, and various options need to be taken into account for ameliorating these effects (e.g., changing optics beam diameter or wavelength, tailoring the flow field, introducing advanced beam control techniques, etc.). Sometimes several approaches must be combined. Random flow fields include turbulent boundary layers and shear layers, the latter being developed at a flow discontinuity (e.g., fuselage protuberance or recession). As reported in [153], the optical aberrations impressed on a beam transiting these random flows are determined by three aerodynamic parameters: the field fluctuating density $\rho$, the correlation length along the optical axis $l_{z}$, and the total path length through the disturbance $L$. The predicted wave-front variance, assuming isotropic turbulence, is given by:

$$
\sigma^{2}=2 G^{2} \int_{0}^{L}\left\langle\rho^{\prime 2}\right\rangle l_{z} d z \quad \text { for } l_{z} \ll L
$$

with $G$ being the Gladstone-Dale parameter [142], relating medium index of refraction to density fluctuations. If the condition $l_{z} \ll L$ is not fulfilled (e.g., in the case of shear layers), then the eq. (24) overestimates the actual aberrations. In general, the complexity of the AO field for an airborne system increases with both the Mach number $(M=V / a$ where $V$ is the fluid velocity and $a$ the velocity of sound) and Reynolds number (Re= $V W / v$ where $W$ is the size of flow pattern and $v$ is the kinematic viscosity). For velocities below $M=0.3$ compressibility effects are negligible and there are little optical aberrations. As $M$ increases beyond 0.3 , compressibility effects become important. The flow velocity, which is zero at the wall (airborne platform surface), and increase away from the wall to the free-stream velocity. The ensuing acceleration yields rotational flow near the surface, leading to either steady or unsteady density variations. The flow is heated as it is compressed and accelerated from the free-stream velocity to stagnation at the wall. Assuming an adiabatic process, this heating can be approximated by [153]:

$$
T_{w}=b T_{0}\left[1+r \frac{(\gamma-1)}{2} M_{0}^{2}\right]
$$

where $T_{w}$ is the adiabatic wall temperature, $T_{0}$ is the free-stream temperature, $M_{0}$ is the free-stream Mach number, $r$ is the recovery parameter ( $r=0.85$ laminar, 0.89 turbulent), $\gamma$ is the adiabatic index (ratio of specific heat at constant pressure to that at constant volume). For $M_{0}<6, b \approx 1.0$; for $M_{0}>8, b \approx 0.5$. As $M_{0}$ continues to increase, the fluid temperature and velocity changes induce increasing density changes. In the transonic regime $(0.8 \leq M \leq 1)$, local supersonic flows (in which shocks appear) cause additional reflection and refraction effects. In the supersonic regime $(1 \leq M$ $\leq 8)$ the high stagnation temperatures can cause thermo-optical window distortions (in some cases a coolant is needed). In the transonic regime $(M>8)$ dissociation and ionisation result from the gas heating. These plasmas introduce another level of optical distortion, with particularly strong vibrational absorption bands. In this regime, strong coupling between acoustical and turbulence fields occurs [153]. The boundary layers can be either laminar or turbulent, the first being characterised by smooth density variations (except for the case of external heating or cooling of the flow), and the second being characterised by a chaotic flow (although the chaos is known to be statistically definite). Extensive research has demonstrated that the magnitude of the density fluctuations in a turbulent boundary layer is driven by the difference between the density at the wall $\rho_{w}$ and the free-stream density $\rho_{0}[155,156]$. The density at the wall in the regime of no shock waves and assuming zero pressure gradient across the boundary layer is given by [154]:

$$
\rho_{w}=\rho_{0}\left[1+r \frac{(\gamma-1)}{2} M_{0}^{2}\right]
$$

Other relationships have also been developed for evaluating the optical effects for both subsonic flow and transonic/supersonic regimes. For an inviscid flow, the density ratio from one streamline to another (no shock waves) is given by: 
This is the author pre-publication version. This paper does not include the changes arising from the revision, formatting and publishing process. The final paper that should be used for referencing is:

R. Sabatini, M. A. Richardson, A. Gardi, S. Ramasamy, "Airborne laser sensors and integrated systems", Progress in Aerospace Sciences (2015), http: //dx.doi.org/10.1016/j.paerosci.2015.07.002i.

$$
\frac{\rho_{b}}{\rho_{a}}=\left\{\frac{1+r \frac{(\gamma-1)}{2} M_{a}^{2}}{1+r \frac{(\gamma-1)}{2} M_{b}^{2}}\right\}^{\frac{1}{\gamma-1}}
$$

Using this equation and the Gladestone-Dale relationship N'=G $\rho^{\prime}$ (where n' is the index of refraction variation and $\rho$ ' is the density fluctuation), the corresponding optical phase $\Delta \phi$ can be determined, by integrating $\rho^{\prime} \equiv|\rho \mathrm{a}-\rho b|$ through the disturbance in the $\mathrm{z}$ (optical axis) direction:

$$
\Delta \phi=K G \int_{0}^{L} \rho^{\prime} d z
$$

where $\mathrm{K}$ is the wavenumber and $\mathrm{L}$ is the path length through the disturbance. More detailed discussions about inviscid and viscous flows, as well as shock waves effects on airborne systems performance may be found in the literature [22, 25, 26, 27].

\subsubsection{Combined propagation equation}

Based on all the physical phenomena described above, a comprehensive equation is introduced for the propagation of laser radiation in atmosphere. Assuming a Gaussian profile of the laser beam at the source and an average focused irradiance, the comprehensive expression of the peak irradiance, $I_{P}$, accounting for absorption, scattering, diffraction, jitter, atmospheric turbulence and thermal blooming effects is[12]:

$$
I_{P}(z, \lambda)=\frac{b(z) \tau(z, \lambda) P(\lambda)}{\pi\left(a_{d}^{2}(z, \lambda)+a_{j}^{2}(z)+a_{t}^{2}(z, \lambda)\right)}
$$

where $\mathrm{z}$ is the linear coordinate along the beam, $\lambda$ is the wavelength, $P(\lambda)$ is the transmitted laser power, $b$ is the blooming factor, $\tau(z, \lambda)$ is the transmittance coefficient, which accounts for absorption and scattering associated with all molecular and aerosol species present in the path. The $1 / e$ beam radii associated with diffraction, $a_{d}(z, \lambda)$, beam jitter, $a_{j}(z)$, and turbulence, $a_{t}(z, \lambda)$, can be calculated as [12,34]:

$$
\begin{aligned}
a_{d}(z, \lambda) & =\frac{Q z \lambda}{2 \pi a_{0}} \\
a_{j}^{2}(z) & =2\left\langle\Theta_{x}^{2}\right\rangle z^{2} \\
a_{t}(z, \lambda) & =\frac{2 C_{n}^{6 / 5} z^{8 / 5}}{\lambda^{1 / 5}}
\end{aligned}
$$

where $\mathrm{Q}$ is the beam quality factor, ao is the beam 1/e radius, $\left\langle\Theta_{x}^{2}\right\rangle$ is the variance of the single axis jitter angle that is assumed to be equal to $\left\langle\Theta_{y}^{2}\right\rangle$, and $C_{n}{ }^{2}$ is the refractive index structure constant. An empirical model for the blooming factor $b(z)$, which is the ratio of the bloomed $I_{B}$ to unbloomed $I_{U B}$ peak irradiance, is:

$$
b(z)=\frac{I_{B}}{I_{U B}}=\frac{1}{1+0.0625 N^{2}(z)}
$$

$\mathrm{N}$ is the thermal distortion parameter, calculated as:

$$
N(z)=\frac{-n_{T} \alpha_{m} P z^{2}}{\pi d_{0} v_{0} c_{P} a_{0}^{3}} \cdot\left[\frac{2}{z^{2}} \int_{0}^{R} \frac{a_{0}}{a\left(z^{\prime}\right)} d z^{\prime} \int_{0}^{Z \prime} \frac{a_{0}^{2} v_{0} \tau \prime \prime}{a} d z^{\prime \prime}\right]
$$

where $v_{o}$ is the uniform wind velocity in the weak attenuation limit $\left(\gamma_{<} \ll<1\right), n_{T}, d_{o}$, and $c_{p}$ are, respectively, the coefficients of index change with respect to temperature, density, and specific heat at constant pressure. The transmittance coefficient $\tau$ depends on the integral effect of absorption and scattering phenomena, both for molecular and aerosol species, on the entire beam length. The specific wavelengths chosen to represent HF, DF, and CO lasers are those with the best transmission characteristics. Only the mid-latitude winter and mid-latitude summer models were considered. It is interesting to note that for the $\mathrm{CO}_{2}$ wavelength, which is dominated by thermal blooming due to stronger molecular absorption, the peak irradiance is relatively insensitive to both turbulence and aerosol effects. At the shorter wavelengths the effects of turbulence and aerosol attenuation produce wide variations in the peak irradiance. The importance of both aerosol scattering and turbulence effects clearly increases at the shorter wavelengths. The analysis conducted by Gebhardt [34] shows that the near to mid-IR regions offer the best overall transmission characteristics; in particular, the $3.8-\mu \mathrm{m}$ DF wavelength is optimum for varying aerosol and turbulence conditions.

\subsubsection{Computer codes and databases}

Over the last few decades, powerful tools for assessment and exploitation of propagation conditions together with range performance models have been developed. Due to the large number of parameters involved in optical transmission through the atmosphere, numerical calculations of atmospheric transmission are an unavoidable process. The aim of the numerical calculation is to predict with a high degree of accuracy the transmittance through the atmosphere, given a path, atmospheric conditions, wavelength, and a set of measured or predicted meteorological parameters. The computer codes refer to the transmission models or computational techniques implemented in computer programs that generate the transmittance or a transmittance spectrum, given the necessary input by the user. Three numerical methods for atmospheric transmission calculations developed at the Phillips Laboratory, Geophysics Directorate are LOWTRAN (LOW resolution atmospheric TRANsmission), MODTRAN (MODerate resolution atmospheric TRANsmission) and FASCODE (FASt Atmospheric Signature CODE) [157]. The molecular high-resolution transmission code database HITRAN (High Resolution Transmission) is contained in FASCODE, whereas LOWTRAN is a low-resolution transmission code, whereas MODTRAN offers medium resolution. GEISA (Gestion et Etude des Informations Spectroscopiques Atmosphériques: Management and Study of Spectroscopic Information) is another database, which was developed to enable accurate forward radiative transfer calculations using line-by-line and layer-by-layer methods [157]. Once LOWTRAN became available, other computer models more specifically designed to simulate tactical battlefield situations were developed. Such is the case for EOSAEL (Electro-Optical Systems Atmospheric Effects Library) designed by the U.S. Army Atmospheric Sciences Laboratory which is now the U.S. Army Research Laboratory. EOSAEL offers many different modules for calculating the effects of battlefield-induced contaminants like smoke, high explosive dust, and vehicular dust [159]. Various other modules are concerned with the effects of turbulence and with absorption and backscattering of laser radiation, effects of clouds and so on. Other computer codes developed for covering the entire spectrum include LinePak, LBLRTM (Line-By-Line Radiative Transfer Model) and XRTM (X Radiative Transfer Model) [160,_ENREF_147 161]. Some features of the interaction of laser beams with the atmosphere are different than those encountered in routine practice with conventional optical systems. Most of these differences are the result of the interaction of the highly monochromatic laser radiation with the fine structure of the atmosphere. Particularly, molecular absorption and scattering are the dominating attenuation phenomena, both of which are strongly wavelength dependent. Conventional (passive) electrooptical systems typically operate over bandwidths that are large compared to the width of most molecular absorption lines. As a result, the response of passive systems is integrated over the entire band and the effects the fine structure of the atmosphere are 
This is the author pre-publication version. This paper does not include the changes arising from the revision, formatting and publishing process. The final paper that should be used for referencing is:

R. Sabatini, M. A. Richardson, A. Gardi, S. Ramasamy, "Airborne laser sensors and integrated systems", Progress in Aerospace Sciences (2015), http: //dx.doi.org/10.1016/j.paerosci.2015.07.002i.

averaged out. These effects, however, are most severe for (active) laser systems, which typically operate over long ranges and use a naturally occurring atmosphere gas as the laser gain medium. In these cases, there is an unavoidable coincidence of the laser line with an atmospheric absorption line. The fine structure of the atmosphere can also have significantly different effects on the atmospheric transmission of transmit and receive paths of an active system like a LRF or a LIDAR.

\subsubsection{Vertical and oblique path propagation}

The transmittance of the atmosphere for a laser beam propagating along the slant path shown in Fig. 22 is then given by:

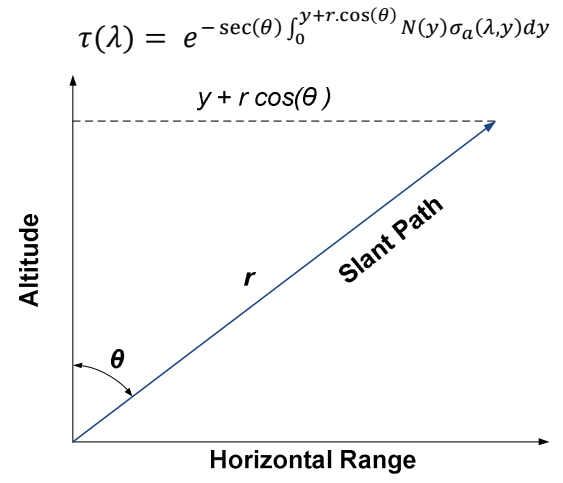

Fig. 22 Geometry of laser beam propagating along slant path.

The ideal gas law may be used to express the integrand in eq. (35) in terms of the atmospheric pressure $p(y)$ at the altitude $y$ and the fractional concentration $f(y)$. Thus, we have:

$$
N(y)=f(y) p(y) / k T(y)
$$

For an isothermal atmosphere and assuming that air is an ideal gas, the pressure as a function of the altitude can be shown to be given by the following expression:

$$
p(y)=p_{0} e^{-y / H}
$$

where $H=k T / m g$ is customarily referred to as the scale height. The parameters $m=\left(\Sigma_{j} m_{j} N_{j}\right) / N$ and $g$ are the average molecular mass and gravitational acceleration, respectively. The scale height is the distance in which the pressure of an isothermal atmosphere of constant composition drops by a factor of $e^{-1}$. Eq. (37) does not apply to the ozone and water vapour concentrations. Assuming that the absorption cross section $\sigma_{a}(f)$ has a Lorentz line shape of line width $\Delta f$ (i.e., collisions are the dominant linebroadening mechanism), then:

$$
\Delta f=\Delta f_{0}\left(\frac{p(y)}{p_{0}}\right)^{\sqrt{\frac{T_{0}}{T(y)}}}
$$

The subscript $O$ refers to a reference altitude. The absorption cross section as a function of frequency and altitude may be expressed in the form:

$$
\sigma_{a}(f, y)=\frac{S(y) \Delta f(y)}{2 \pi\left[\left(f-f_{0}\right)^{2}+\left(\frac{\Delta f(y)}{2}\right)^{2}\right]}
$$

The parameter $S(y)$ is called the line intensity or line strength and is given by:

$$
S(y)=\int_{-\infty}^{\infty} \sigma_{a}(f, y) d f
$$

The total molecular absorption coefficient at the laser frequency $f$ is found by summing over each molecular species present and the various allowed transitions that contribute to the total absorption coefficient. Therefore, we have:

$$
\alpha(f)=\sum_{i} \sum_{j} \frac{s_{i j}(y) \Delta f_{i j}(y) N_{j}(y)}{2 \pi\left[\left(f-f_{0, i j}\right)^{2}+\left(\frac{\Delta f_{i j}(y)}{2}\right)^{2}\right]}
$$

The subscript $i$ refers to the $i^{\text {th }}$ line of the $j^{\text {th }}$ molecular species with the number density $N_{j}(y)$. For example, the He-Ne laser, in addition to the $0.6328 \mu \mathrm{m}$ transition, can also be operated at $1.152276 \mu \mathrm{m}$. Because of the need for high-resolution data it is sometimes desirable to calculate the atmospheric absorption coefficient. Combining eqs. (35) and (41), the atmospheric transmittance at the frequency $f$ is given by:

$$
-\frac{\sec (\theta)}{2 \pi} \int_{0}^{y+r \cdot \cos (\theta)} \sum_{i} \Sigma_{j} \frac{s_{i j}(y) \Delta f_{i j}(y) N_{j}(y)}{2 \pi\left[\left(f-f_{0, i j}\right)^{2}+\left(\frac{\Delta f_{i j}(y)}{2}\right)^{2}\right]} d y
$$

This equation must be evaluated by analytic or numerical techniques. Most calculations of this sort assume a model standard clear atmosphere and require estimates that are at times not much better than educated guesses. Attempts have been made to compile all known molecular absorption lines and theoretically calculate atmospheric absorption coefficients [161]. In particular, a number of empirical relationships for the altitude correction have been experimentally determined for NIR lasers depending on the grazing angles $[12,163]$. The regression resulting from these experimental activities has the general form:

$$
\frac{\gamma_{H}(z)}{\gamma_{0}}=J_{0}+J_{1} \cdot h(z)
$$

where $\gamma_{a t m_{H}}$ is the extinction coefficient of the slant path, $\gamma_{a t m}$ is the extinction measured at ground level (QFE altitude), $h$ is the altitude above ground level (AGL), and $J_{0}, J_{l}$ are linear regression coefficients varying as a function weather conditions and grazing angle.

\subsubsection{Propagation through haze, fog and rain}

The effects of precipitation (rain, fog, and snow) on the transmission of laser beams were studied extensively [164]. A summary of their results is reproduced in Fig. 24. The figure shows the attenuation coefficients for a typical dense fog and a representative shower for the wavelength range from $0.6 \mu \mathrm{m}$ to 15 $\mu \mathrm{m}$. The absorption of a layer of water containing the same amount of liquid water as the rain is also presented. For the typical wavelength range considered, the attenuation by rain, which increases gradually from the visible region to the $I R$ region, is considerably less than that for fog. For dense fogs, Chu and Hogg found that the attenuation at $10.6 \mu \mathrm{m}$ can exceed $40 \mathrm{~dB} / \mathrm{km}$. It is interesting to note that attenuation by light fog at $\lambda=3.5 \mu \mathrm{m}$ and $\lambda=10.6 \mu \mathrm{m}$ is up to one order of magnitude less than at 0.63 $\mu \mathrm{m}$ (He-Ne laser), while attenuation of $0.63 \mu \mathrm{m}$ wavelength radiation in rain is about $20 \%$ less than at $3.5 \mu \mathrm{m}$.

In Fig. 23, absorption for a water layer containing the same amount of liquid water as the rain is represented by the solid curve. The attenuation properties of snow were reported being between those of fog and rain [165]. Due to the limited knowledge of the exact atmospheric composition at any instant, it is not possible to univocally determine the transmittance and the derived measurable such as the operational range and resolution of an electro-optical system by directly inverting the linear and nonlinear propagation models introduced above. It is therefore essential to review the engineering approach to atmospheric modelling. An empirical model was introduced by Elder and Strong [ES] and modified by Langer [4], and related the 
This is the author pre-publication version. This paper does not include the changes arising from the revision, formatting and publishing process. The final paper that should be used for referencing is:

R. Sabatini, M. A. Richardson, A. Gardi, S. Ramasamy, "Airborne laser sensors and integrated systems", Progress in Aerospace Sciences (2015), http: //dx.doi.org/10.1016/j.paerosci.2015.07.002i.

transmittance in the $i^{\text {th }}$ atmospheric window to the Relative Humidity $(R H)$. The assumption is that only the water content of the air is affecting the atmospheric transmittance, and specifically that the concentration of $\mathrm{H}_{2} \mathrm{O}$ influences the absorption, whereas the size and number of water droplets influences the scattering. For these reasons this assumption is accurate within the atmospheric windows. The model introducing the dependence of the scattering coefficient on the rainfall rate was introduced by Middleton. Based on the Elder-Strong Langer Middleton (ESLM), analytical expressions of the transmittances were developed for all the atmospheric windows in the infrared spectrum. These expressions depend on a number of additional parameters such as atmospheric visibility, precipitation and fog. By introducing the total condensed water along the laser beam path, $w$, the meteorological visibility, $V$, and the rainfall rate $\Delta x / \Delta t$, the estimated atmospheric transmittance values in the one-way case [52]:

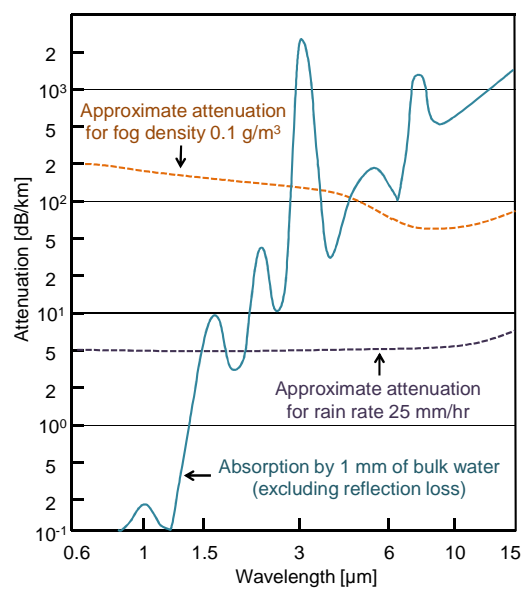

Fig. 23. Attenuation of infrared radiation due to rain (lower dashed curve) and fog (upper dashed curve).

Table 5

Atmospheric transmittance for horizontal paths at sea level.

\begin{tabular}{|c|c|}
\hline Conditions & Transmittance \\
\hline $\begin{array}{c}\mathrm{V} \geq 6 \mathrm{~km} \\
w \geq w_{i}\end{array}$ & $k_{i}\left(\frac{w_{i}}{w}\right)^{\beta_{i}} \exp \left[-\frac{3.91}{V} R\left(\frac{\lambda_{i}}{0.55}\right)^{-(0.0057 \cdot V+1.025)}\right]$ \\
\hline $\begin{array}{c}\mathrm{V} \geq 6 \mathrm{~km} \\
w<w_{i}\end{array}$ & $\exp \left\{-R\left[A_{i} \sqrt{w_{t}}+\frac{3.91}{V}\left(\frac{\lambda_{i}}{0.55}\right)^{-(0.0057 \cdot V+1.025)}\right]\right\}$ \\
\hline $\begin{array}{c}\mathrm{V}<6 \mathrm{~km} \\
w \geq w_{i}\end{array}$ & $k_{i}\left(\frac{w_{i}}{w}\right)^{\beta_{i}} \exp \left[-\frac{3.91}{V} R\left(\frac{\lambda_{i}}{0.55}\right)^{-0.585 \sqrt[3]{V}}\right]$ \\
\hline $\begin{array}{c}\mathrm{V}<6 \mathrm{~km} \\
w<w_{i}\end{array}$ & $\exp \left\{-R\left[A_{i} \sqrt{w_{t}}+\frac{3.91}{V}\left(\frac{\lambda_{i}}{0.55}\right)^{-0.585 \sqrt[3]{V}}\right]\right\}$ \\
\hline $\begin{array}{c}\text { Rain } \\
w \geq w_{i}\end{array}$ & $k_{i}\left(\frac{w_{i}}{w}\right)^{\beta_{i}} \exp \left[-0.365 R\left(\frac{\Delta x}{\Delta t}\right)^{0.63}\right]$ \\
\hline $\begin{array}{c}\text { Rain } \\
w<w_{i}\end{array}$ & $\exp \left\{-A_{i} \sqrt{w}\left[-0.365 R\left(\frac{\Delta x}{\Delta t}\right)^{0.63}\right]\right\}$ \\
\hline
\end{tabular}

Table 6

Coefficients for the atmospheric transmittance.

\begin{tabular}{|c|c|c|c|c|}
\hline Constant & $\boldsymbol{A}_{\boldsymbol{i}}$ & $\boldsymbol{k}_{\boldsymbol{i}}$ & $\boldsymbol{\beta}_{\boldsymbol{i}}$ & $\boldsymbol{w}_{\boldsymbol{i}}$ \\
\hline I & & & & \\
\hline II & 0.0305 & 0.800 & 0.112 & 54 \\
\hline III & 0.1303 & 0.765 & 0.134 & 54 \\
\hline IV & 0.211 & 0.830 & 0.093 & 2.0 \\
\hline V & 0.350 & 0.814 & 0.111 & 1.1 \\
\hline VI & 0.373 & 0.827 & 0.095 & 0.35 \\
\hline
\end{tabular}

\subsubsection{Propagation through atmospheric turbulence}

Temperature fluctuations are introduced into the atmosphere by relatively large-scale phenomena as convection from solar heating of the earth surface and by wind shear. The turbulence energy is thus injected over scale sizes larger than some minimum value $L_{0}$, which is called the outer scale of turbulence. The corresponding wave number is $K_{0}=\left(2 \pi / L_{0}\right)$. For wave numbers smaller than $K_{0}$, the form of the turbulence energy spectrum is determined by local surface terrain and weather conditions. Since conditions found at one point in the atmosphere usually differ from those that exist at another point, the turbulence is generally not homogeneous and isotropic. Unfortunately, the only situation for which there is a well-developed atmospheric model is in the case of homogeneous isotropic turbulence, where the refractive index fluctuations are random due to well-mixed temperature fluctuations. Typical scale sizes for $L_{0}$ are about $100 \mathrm{~m}$, or 0.2 times the height above the ground, whichever is less. The inner scale of turbulence $l_{0}$ marks the turbulence scale sizes at which viscous dissipation converts the turbulent energy into heat. Typically it is on the order of $1 \mathrm{~mm}$ near the ground to about $1 \mathrm{~cm}$ at the tropopause. The corresponding wave number is $K_{m}=\left(2 \pi / l_{0}\right)$. The region between $K_{o}$ and $K_{m}$ is known as the inertial sub-range. The motion of the large turbulent eddies, or cells, causes the formation and transfer of energy to smaller eddies that transfer their energy to still smaller eddies. This process continues until viscous effects dominate and the turbulent energy of the smallest eddies is dissipated. The viscous dissipation begins at the spatial wave number $K_{m}$. Intuitively, $l=2 \pi / \mathrm{K}$ may be regarded as the size of the turbulent eddy, or cell. The turbulent inner and outer scales are by no means fixed, constant values, but possess dynamic vertical profiles that depend on such factors as temperature, pressure, humidity, and wind speed [152].

\subsubsection{Refractive index variations}

The single most important parameter appearing in almost all equations that describe beam disturbances caused by turbulence is the refractive index structure coefficient $C_{n}$. It is governed by the pressure and temperature difference at two points separated by the distance $r$ (measured in centimetres) and may be approximated by [152]:

$$
C_{n}=\left[79.10^{-6} \frac{p}{T^{2}}\right] C_{T}
$$

where the temperature structure parameter is:

$$
C_{T}=\sqrt{\left\langle\left(T_{1}+T_{2}\right)^{2}\right\rangle} \frac{1}{\sqrt[3]{r}}
$$


This is the author pre-publication version. This paper does not include the changes arising from the revision, formatting and publishing process. The final paper that should be used for referencing is:

R. Sabatini, M. A. Richardson, A. Gardi, S. Ramasamy, "Airborne laser sensors and integrated systems", Progress in Aerospace Sciences (2015), http: //dx.doi.org/10.1016/j.paerosci.2015.07.002i.

The temperatures $T, T_{1}$, and $T_{2}$ are all in $\mathrm{K}$, and $p$ is the atmospheric pressure in millibars. Typical values are: for strong turbulence, $C_{n}=5 \times 10^{-7}$; for intermediate turbulence, $C_{n}=4 \times 10^{-}$ ${ }^{8}$; and for weak turbulence, $C_{n}=8 \times 10^{-9}$. The structure parameter usually appears in the literature in the form $C_{n}{ }^{2}$, which varies from about $10^{-17} \mathrm{~m}^{-2 / 3}$ for extremely weak turbulence to $10^{-13} \mathrm{mn}^{-2 / 3}$ or more when the turbulence is strong. This latter value is usually observed near the ground in direct sunlight. Measurements of $C_{n}$ with temperature sensors $1.6 \mathrm{~m}$ above the ground have shown that the minimum value of $C_{n}$, occurs about one to two hours before sunrise and after sunset. The peak values have been recorded around noon on sunny days. It is evident that the structure parameter $C_{n}$ decreases with altitude. Some approximate values are given in Table 7.

Table 7

Typical values of $C_{n}$ as a function of height.

\begin{tabular}{|c|c|}
\hline Height $(\mathbf{K m})$ & $C_{n}\left(\mathbf{m}^{\mathbf{- 1 / 3}}\right) \mathbf{x} \mathbf{1 0}^{\mathbf{8}}$ \\
\hline 0.001 & 30 \\
0.003 & 20 \\
0.01 & 15 \\
0.03 & 10 \\
0.1 & 6 \\
0.3 & 4 \\
1.0 & 1 \\
3.0 & 1 \\
\hline
\end{tabular}

Various models have been suggested to describe $C_{n}{ }^{2}$ through the altitude $(h)$ only. Most of these models can be condensed by the following general formula:

$$
C_{n}{ }^{2}(h)=C_{n o}{ }^{2} h^{-b} \exp \left(-h / h^{\prime}\right)
$$

where $C_{n o}{ }^{2}, b$ and $h$ are the chosen parameters. Particularly, the following models can been found in the literature.

Fried model [166]:

$$
\begin{aligned}
b=1 / 3, h^{\prime}=3200 m, C_{n o}{ }^{2}=4.22 \times 10^{-14} \mathrm{~m}^{-1 / 3} \\
C_{n}{ }^{2}(h)=4.22 \times 10^{-14} h^{-1 / 3} \exp \left(-\frac{h}{3200}\right) m^{-2 / 3}
\end{aligned}
$$

Brookner model [167]:

$$
\begin{aligned}
b=5 / 6, h^{\prime}=320 m, C_{n o}{ }^{2}=3.6 \times 10^{-13} \mathrm{~m}^{-1 / 6} \\
C_{n}{ }^{2}(h)=3.6 \times 10^{-13} h^{-5 / 6} \exp \left(-\frac{h}{320}\right) m^{-2 / 3}
\end{aligned}
$$

Tatarski model [168]:

$$
\begin{aligned}
b=4 / 3, h^{\prime}=\infty, C_{n o}{ }^{2} & =4.16 \times 10^{-13} \mathrm{~m}^{-2 / 3} \\
C_{n}{ }^{2}(h) & =4.16 \times 10^{-13} h^{-4 / 3} \mathrm{~m}^{-2 / 3}
\end{aligned}
$$

Another model, accounting also for wind influence, is the Hufnagel model presented below [169]:

$$
\begin{array}{r}
C_{n}{ }^{2}(h)=2.7 \times 10^{-16}\left[2.2 \times 10^{-37} h^{10}\left(\frac{w}{27}\right)^{2} \times \exp \left(-\frac{h}{1000}\right)+\right. \\
\left.\exp \left(-\frac{h}{1500}\right)\right] m^{-2 / 3}
\end{array}
$$

In the Hufnagel model, the factor $w$ is the average wind speed in the range of elevations from 5 to $20 \mathrm{~km}$. The Hufnagel model is only valid for the range of elevations from $3 \mathrm{~km}$ above the local ground level to $24 \mathrm{~km}$ above sea level. The Tatarski model is a theoretical model while the other models are based on experimental measurements. All models have been checked under different weather conditions and measurements fields. Tatarski model appears to fit best for small altitudes, while the other models are more suited to higher altitudes $[170,171]$. It must be mentioned, however, that some $C_{n}{ }^{2}(h)$ vertical profile measurements performed at night [172], indicate increases in $C_{n}{ }^{2}(h)$ at altitudes on the order of 10 to $20 \mathrm{~km}$.

\subsubsection{Turbulence effects}

Fig. 24 illustrates an expanded beam is transmitted through a region of atmospheric turbulence to a target where it is observed.

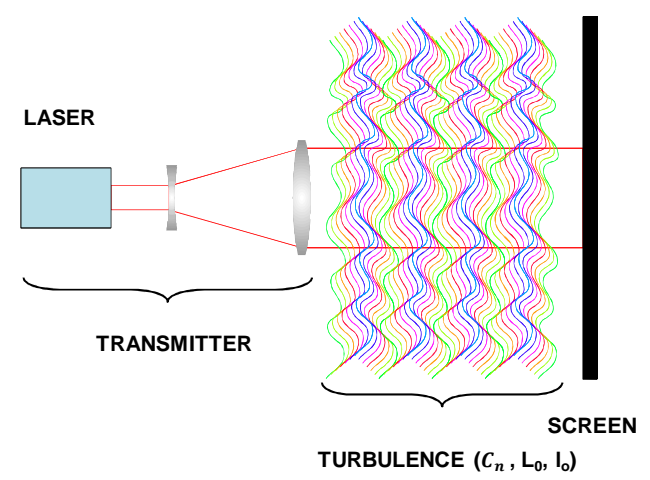

Fig. 24. Transmission of a laser beam through atmospheric turbulence.

The region is characterized by the refractive index structure coefficient $C_{n}$, the outer scale $L_{0}$ and the inner scale $l_{0}$. The characteristics of the transmitter are determined by the output beam diameter $d$, the wavelength $\lambda$, and the focal length $f$ of the beam expander. The turbulence present is described by outer and inner scale sizes as well as the refractive index structure coefficient $C_{n}$. Depending on the dominant turbulence scale size and beam diameter, two cases that lead to different effects can be identified. For instance, if the scale sizes at all points along the beam path are larger than the beam diameter, the turbulence cells act as very weak lenses that deflect the beam as a whole in a random way, leaving the beam diameter essentially unaltered. This is shown schematically in Fig. 25. The resulting beam displacement from the initial beam direction is known as "beam wander".

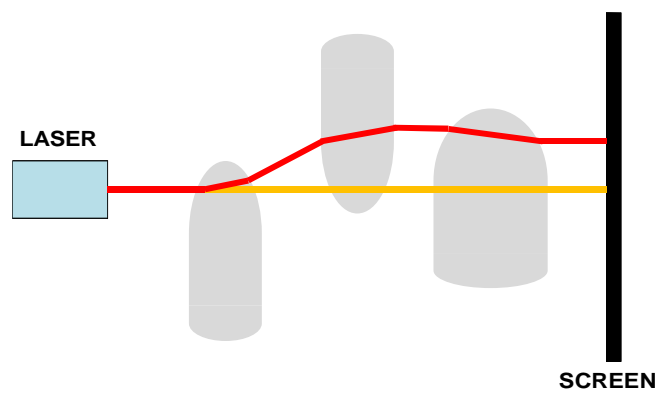

Fig. 25. Laser beam deflected by turbulence cells larger than the beam diameter. 
This is the author pre-publication version. This paper does not include the changes arising from the revision, formatting and publishing process. The final paper that should be used for referencing is:

R. Sabatini, M. A. Richardson, A. Gardi, S. Ramasamy, "Airborne laser sensors and integrated systems", Progress in Aerospace Sciences (2015), http: //dx.doi.org/10.1016/j.paerosci.2015.07.002i.

When the scale sizes (or cell dimensions) are smaller than the diameter of the beam, diffraction and refraction of the beam takes place and the beam's intensity profile is smeared out and as a rule markedly distorted. This effect, schematically illustrated in Fig. 26 , is referred to as "beam breathing" and "scintillation". Depending on the characteristics of the turbulence and of the laser beam, both cases may be observed simultaneously.

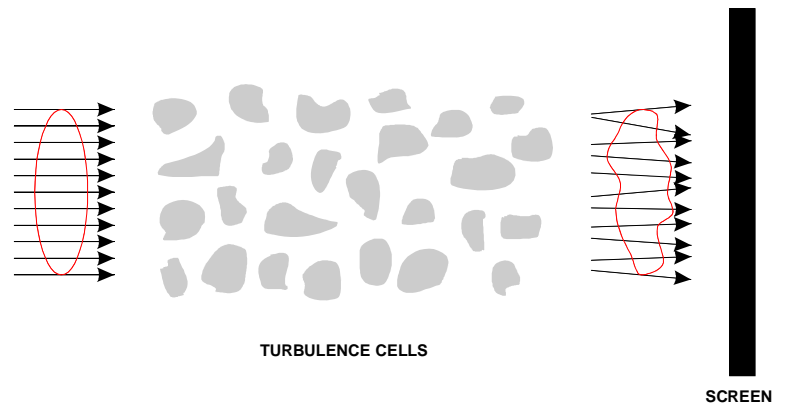

Fig. 26. The laser beam is broken up by turbulence cells smaller than the beam diameter.

\subsubsection{Turbulence-induced beam wander}

The term beam wander is used to describe the rapid displacement of the beam spot on a screen a distance $\mathrm{L}$ from the laser. Since this effect is due to large turbulence scale sizes, a geometrical optics approach can be adopted. The expression for the radial variance of beam wander is given by [173]:

$$
\sigma_{r}^{2}=1.90 . C_{n}^{2} \cdot \frac{L^{3}}{2 w_{0}}
$$

where $w$ is the beam spot size at the transmitter, $L$ is the screen distance from the laser and $C_{n}$ is the index structure coefficient. Sometimes it is more desirable to know the relationship between the radial variance $\sigma_{r}{ }^{2}$ and the beam's wavelength $\lambda$. According to Weichel [152], eq. (42) can be rewritten as:

$$
\sigma_{r}^{2}=1.83 . C_{n}^{2} \cdot \sqrt[6]{\frac{L^{17}}{\lambda}}
$$

where both $\lambda$ and $L$ are expressed in meters. Beam wander has been measured under varying conditions of transmitter configurations and turbulence strengths. In general, the results show that beam wander is to a very high degree independent of wavelength. Beam wander becomes a major problem whenever a laser beam must remain on a specific target point for some time. It is possible, however, to negate wandering with the aid of a fasttracking (a few Hertz) optical system. For communication systems it may be more practical to use sufficiently large apertures that will cover the entire range of the beam motion.

\subsubsection{Turbulence-induced beam intensity fluctuations}

The movement of small index-of-refraction inhomogeneities through the path of a beam causes random deflection and interference between different portions of the wavefront, which can lead to an internal breaking up of the beam spot into smaller "hot spots". The resulting beam shape and energy distribution may be considerably different from the ones originated at the laser source. Various experiments performed have shown that the dominant size of the hot spots in the laser pattern observed on a screen distant $L$ from the source is given approximately by $\sqrt{L \lambda}$. Bright patches of about $1 \mathrm{~cm}$ diameter are typical for $L \approx 1 \mathrm{~km}$. Because of the constantly changing pattern, a small detector placed in the beam will measure intensity fluctuations or scintillation. The temporal frequency of the intensity fluctuations recorded fixed point within the beam usually varies between 1 and $100 \mathrm{~Hz}$ [174]. Beam intensity fluctuations are primarily produced by cells that are smaller than the beam diameter. As a result, diffraction plays an important role in scintillation.

\subsubsection{Turbulence-induced beam spreading}

Due to turbulence the beam will also spread in size beyond the dimensions attributed to the usual diffraction spreading. According to the diffraction theory, a focused beam from a uniformly illuminated circular aperture of diameter $d$ produces a radiation pattern in the focal plane whose diameter is:

$$
d_{0}=\frac{\lambda}{d}
$$

Where $f$ is the focal length of the optical system and $\lambda$ the wavelength of the beam. The presence of atmospheric turbulence, however, prevents us from achieving diffraction-limited focal spot sizes. In practice the observed spot sizes are often twice as large as the spot sizes predicted by diffraction theory. The diffraction-limited (ideal) beam diameter and the experimentally observed diameter are plotted against transmitter aperture. The turbulence-induced beam spreading has been shown by Yura [17] to be given in the far-field by:

$$
a_{t} \cong \frac{2 z}{k \rho_{0}}
$$

where $a_{t}$ is the distance from the $z$ axis to where the relative mean irradiance is down by $1 / \mathrm{e}$, and:

$$
\rho_{0}=\frac{1}{\sqrt[5]{\left(.545 . k^{2} C_{n}{ }^{2} z\right)^{3}}}
$$

is a measure of the lateral coherence length of a spherical wave propagating through a medium containing homogeneous turbulence.

\subsection{Laser scattering and target cross section}

Our present understanding of radiation theory does not permit an analytical description, in closed form, of the exact relationship between the radiation emitted by a source (whether natural or artificial) and the radiation received by a remote sensor after having been reflected by object under surveillance. There are well known laws to describe the simple case of an electromagnetic wave incident upon a perfectly planar interface between two media. In this case, the reflected wave depends upon the radiation wavelength, the angle of incidence, and the physical properties (permittivity, permeability and conductivity) of the two adjoining media. The laws governing such a case are sufficiently understood so that the refractive index and extinction coefficient of materials involved may be found by determining the reflection coefficients of the materials. For the more complicated case involving a surface with periodic or random surface irregularities, an analytic determination of the properties of the reflected electromagnetic field may only be approximated. In the past ten years, many papers have been published on scattering, or reflection from rough surfaces. Many theories have been developed, but none is both general and rigorous at the same time. To perform reasonably simple numerical calculations on the basis of these theories, certain simplifying assumptions are introduced, usually including one or more of the following: 
This is the author pre-publication version. This paper does not include the changes arising from the revision, formatting and publishing process. The final paper that should be used for referencing is:

R. Sabatini, M. A. Richardson, A. Gardi, S. Ramasamy, "Airborne laser sensors and integrated systems", Progress in Aerospace Sciences (2015), http: //dx.doi.org/10.1016/j.paerosci.2015.07.002i.

- The dimensions of scattering elements of the rough surface are either much smaller or much greater than the wavelength of the incident radiation.

- The radii of curvature of the scattering elements are much greater than the wavelength of the incident radiation.

- Shadowing or obscuration effects occurring at the surface may be neglected.

- Only the far field is to be considered.

- Multiple reflections may be neglected.

- Consideration is restricted to a particular model of surface roughness (e.g., saw-tooth, sinusoidal protrusions of definite shape and in random position, with random variations in height given by their statistical distribution and correlation function).

Electromagnetic scattering theory has been used in the past to compute radiation backscattering from targets in the microwave region of the spectrum, where the radiation wavelength is much greater than the minute irregularities of the target surface, and where the conductivity of the target material is infinite. In the optical region, where materials have finite conductivity and the surface irregularities have a wide range in size relative to the radiation wavelength, present electromagnetic scattering theory is applicable to only a few special cases, so the only way to determine reflectance in this region for target and background objects is by experimentation.

\subsubsection{Dependence on target area}

The effective target cross-section is defined as:

$$
\sigma=\frac{4 \pi}{\Omega} \rho d A
$$

where $\Omega$ is the scattering solid angle of target (sr), $\rho$ is the target reflectivity and $d A$ is the target area. Both specular and diffuse reflection components may be considered. However, in practice, physicists tend to replace $\Omega$ with the value associated with the standard scattering diffuse target (Lambertian target) having a solid angle of $\pi$ steradians. Thus, eq. (6) reduces to:

$$
\sigma=4 \rho_{T} d A
$$

The cross-sectional area of a laser beam transmitted by a circular aperture from a distance $R$, is given by:

$$
d A=\frac{\pi R^{2} \theta_{T}^{2}}{4}
$$

\subsection{Military systems operational considerations}

Global requirements for mission planning with a particular laser designation system may be initially established by examining the LTD and LGW operating slant ranges required to successfully perform the mission (e.g., optimal delivery of a particular laser weapon). Mission planning with a particular LTD system must have an operational input that factors in the slant-ranges expected for various types of delivery tactics. Mission planning to determine the optimal weapon release point involves a number of factors, including the post-release designation manoeuvre to be employed, the maximum slant range at weapon impact, the target size, laser system error budget, laser power, etc.

\subsubsection{Airborne mission geometry}

Considering the LTD/LGW attack geometry in Fig. 27, the maximum range performance of an LTD/LGB combination can be estimated using:

$$
M D E D=\frac{4 \rho_{T} U A \cos \theta_{t} \cos \theta_{r} \cos \gamma_{R} \tau_{a t m}}{\pi^{2}\left(D_{L}+\alpha_{T} R_{T}\right)^{2} R_{R}{ }^{2}}
$$

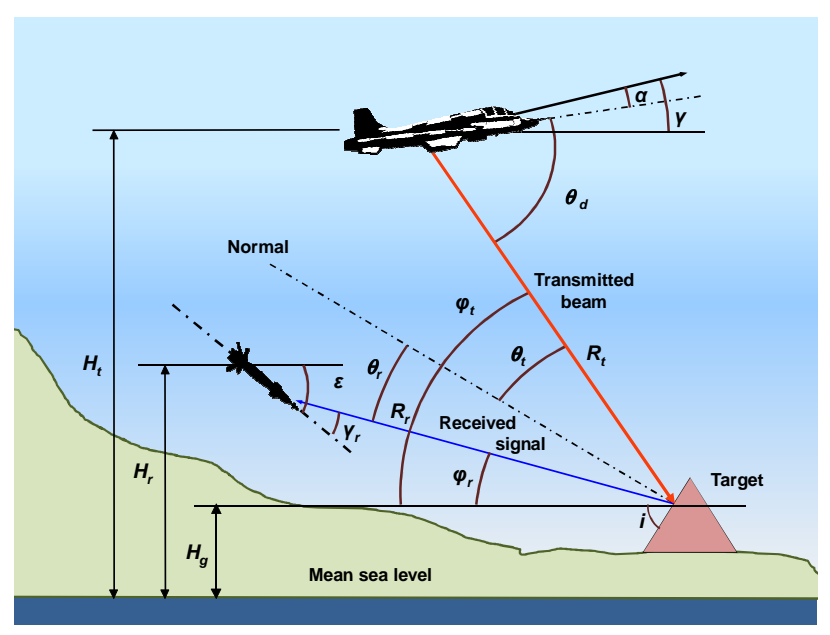

Fig. 27. LTD/LGW mission geometry (vertical profile).

There are three cosine factors in eq. (56). They are related to the assumption of a Lambertian reflection (i.e., diffuse reflection of the laser signal incident on the target surface). It is important, in order to determine the performance of an LTD/LGW combination during an attack, to take into account the variations of the angles $\theta_{t}, \theta_{r}$ and $\gamma_{r}$. On the other hand, in order to calculate the maximum range for an effective illumination in the worst geometric case, it is important to determine the maximum values assumed by these angles during the attack. Moreover, for mission planning purposes, it is useful to express the angles $\theta_{t}, \theta_{r}$ and $\gamma_{r}$ as functions of other physical or geometrical parameters that are known prior the mission (e.g., seeker FOV, target inclination, etc.). The maximum theoretical value of the angle $\gamma_{r}$ can be determined as a function of the seeker Minimum Detectable Energy Density (MDED). However, we must consider that the seeker of the LGW must always intercept a portion of the reflected signal sufficient to produce a response of the detector in order to guide the weapon against the target. In other words, the angle $\gamma_{r}(M D E D)$ should always be greater than the FOV of the seeker. For the purpose of determining the maximum values that the angles $\theta_{t}$ and $\theta_{r}$ can reach during an attack, which determine the absolute minimum performance of a particular LTD/LGB combination (worst case), it is meaningful to take into account the tactics of typical self-designation attacks illustrated in Fig. 28. Since the designation is initiated in the final portion of the bomb trajectory (i.e., with an LTD-target range typically between 1.2 and 2.0 times the release range), it is generally performed at a considerable range from the target. This means that, normally, the angles $\theta_{t}$ and $\theta_{r}$ never reach values close to $90^{\circ}$ during an attack, even in the worst case when $i=90^{\circ}$. On the other hand, in the case of horizontal target $\left(i=0^{\circ}\right)$, the cases where $\theta_{t}$ and $\theta_{r}$ are close to $90^{\circ}$ are of little practical interest. 
This is the author pre-publication version. This paper does not include the changes arising from the revision, formatting and publishing process. The final paper that should be used for referencing is:

R. Sabatini, M. A. Richardson, A. Gardi, S. Ramasamy, "Airborne laser sensors and integrated systems", Progress in Aerospace Sciences (2015), http: //dx.doi.org/10.1016/j.paerosci.2015.07.002i.

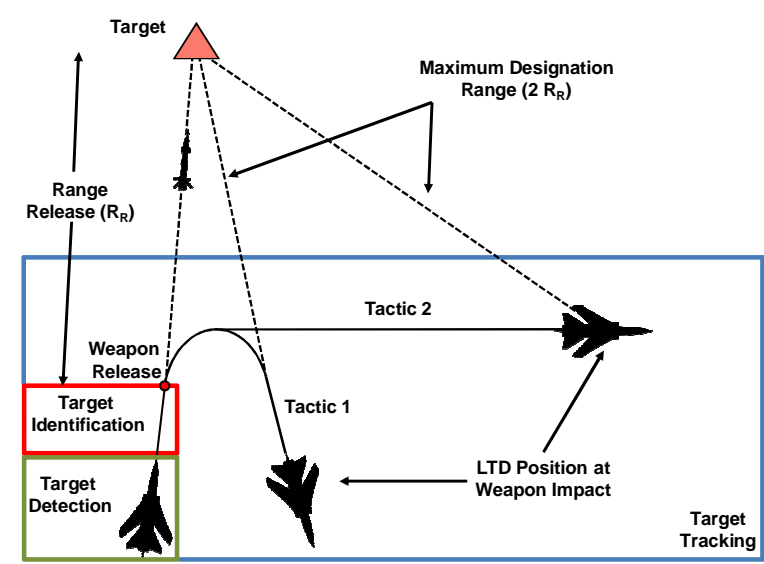

Fig. 28. LTD/LGB mission horizontal profiles (self-designation).

\subsubsection{Error sources}

\subsubsection{Laser spot spillover}

Several characteristics of the laser beam must be tightly controlled if the beam is to be maintained on the desired target surface. First, the laser beam spot should be smaller than the target face. As the LTD produces a beam that diverges as it propagates along the path between the laser and the target, beam spillover effects often degrade weapon accuracy both when designation is performed by a ground LTD or an airborne LTD (Fig. 29).

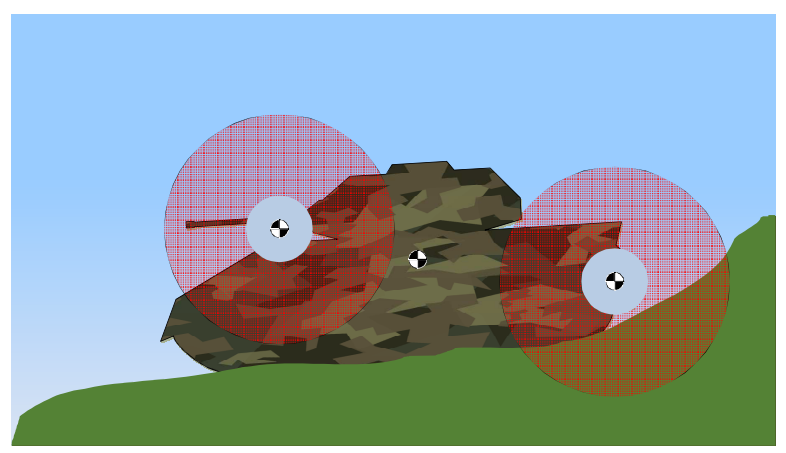

Fig. 29. Laser spot spillover.

Laser beam divergence should therefore be accounted, and appropriate terminal slant-ranges and grazing angles should be chosen such that the spot elongation will not cause spillover around the target.

\subsubsection{Laser spot jitter}

Laser spot jitter is defined as the high frequency motion of the laser spot on a pulse-to-pulse basis, usually of low amplitude, and ostensibly due to minute flexures of the optical bench caused by aircraft vibration. These rapid angular movements of the beam degrade weapon accuracy only slightly when the laser beam is normal to the target face. However, at shallow grazing angles and large slant-ranges, jitter may cause each spot to move hundreds of feet in relation to the aim point and in relation to the previous spot location. In many cases (e.g., most self-designation LGB deliveries), this movement is near perpendicular to the weapon flight path and create false left-right commands. Therefore, as the weapon manoeuvres to intercept the moving spot, this factor may cause rapid depletion of the LGB available energy and may cause large miss distances to be generated.

\subsubsection{Laser boresight error}

Laser boresight error is defined as the misalignment between the location of the aiming reticle and the laser spot on the target. This error is easy to visualize as a geometric progression of the beam wandering away from the sensor sight line as the range increases. Boresight error is not only a static error source but can be a dynamic error as well. The system optical bench may distort, changing the designator/sensor boresight relation as the system is slewed through its field of regard. In addition, manoeuvring ( $\mathrm{g}$ forces) may cause additional shifts as the structure between the designator and sensor deflects under load. In some cases, particularly at long slant ranges, boresight error can place the laser spot off the target, resulting in a weapon miss. If the magnitude of boresight error is known, however, the aim point can be shifted to compensate.

\subsubsection{Laser pointing error}

Laser pointing error is due to the inability to place the laser spot at the exact desired location on the target. This is usually observed when trying to designate a small target from long ranges, where the reticule size can obscure the target. If the sensor magnification of the target is insufficient, it is difficult to know exactly where the aiming reticule is located on the target and, sometimes, it may be also difficult to know if it is on the target at all.

\subsubsection{Tracking error}

Tracking error is a generic term that encompasses other forms of spot movement from the desired aim point. Where jitter is a random movement of the beam around a central axis, tracking error may be described as undesired movement of this central axis around or away from the aim point. This movement of the central beam axis may or may not be visible to the operator depending on the magnitude of the error and the quality of the sensor presentation to the operator. At long slant-ranges, automatic tracking systems can exhibit beam wander that overwhelms other sources of error. This wander is caused by movement of the video tracking gates on-or-about the aimpoint as the viewing aspect changes. The changing aspect or look angle produces changes in the aim point contrast with respect to its background. This, in turn, varies the location of the contrast driven tracking gate position with a consequent shift in beam position. Other causes for tracking error may include $\mathrm{g}$ forces (mentioned earlier), transient angle rate errors due to rapid bank angle changes, or momentary errors due to LOS masking. Motion of the laser spot during the last three seconds prior to impact may induce unnecessary corrections to the weapon flight and result in a miss.

\subsubsection{Podium effect}

For an LGB to guide, the seeker must be in a position to receive the reflected laser energy. During a self-designation attack against a vertical target, there is a risk that the laser spot will move around the target face relative to the weapon LOS, as the designator aircraft flies the recovery manoeuvre, and that the weapon will not receive the reflected laser energy during the final critical moments before impact. This phenomenon, known as the "podium effect", is particularly apparent when the designator to target line is significantly different to that of the weapon's flight path. To avoid the podium effect, the designating aircraft should 
This is the author pre-publication version. This paper does not include the changes arising from the revision, formatting and publishing process. The final paper that should be used for referencing is:

R. Sabatini, M. A. Richardson, A. Gardi, S. Ramasamy, "Airborne laser sensors and integrated systems", Progress in Aerospace Sciences (2015), http: //dx.doi.org/10.1016/j.paerosci.2015.07.002i.

maneuver such that the target face is always in front of the aircraft and that the appropriate terminal slant range/angle occurs at weapon impact. This problem can often be eliminated by lasing on top of a horizontal target.

\subsubsection{Sensor resolution}

The size of the target must also be factored against the resolution abilities of the sensor element (FLIR and/or TV) to determine the maximum usable delivery slant range. This will ensure that the operator will be able to resolve the target at a range that is in excess of the maximum range capability of the weapon. This excess or redundant range requirement is necessary to properly detect and then identify the target prior to weapon release. This target detection and identification requirement prior to release has become of almost paramount importance in punitive or other high visibility actions where the blind launches required by other weapon systems prevent their use. The maximum slant-range from which a designator is intended to be operated must be determined as part of the mission planning process as a function of target size, laser system error budget, and laser power. In addition, an attempt should be made to determine what additional range should be selected in order for the target to be properly identified prior to weapon release. This requires an estimate of the time required to first detect the target on the sensor set and then add the time required to fully resolve the target for a positive identification. With current TV/FLIR technologies and good initial cueing, it is usually estimated that at least ten seconds are required to detect the target. Further five to ten seconds are then required to properly identify the target itself.

\subsubsection{Masking}

Another important problem with airborne laser systems is "masking" of the equipment field of regard caused by the aircraft structure and loads (e.g., weapons, external tanks, etc.). Although masking can be reduced/eliminated by a careful aircraft/system design in the case of embedded systems, this is generally a very important constraint for operations with podded systems. A useful way of characterising systems masking characteristics is the so called "Masking Matrix". This is a Cartesian coordinate system in which (most conveniently) azimuth and elevation are plotted for the equivalent FOV of the system. This is given by intersection of the system "visibility matrix" and the "aircraft matrix" (e.g., an aircraft/loads CAD model). An example is the system in Fig. illustrating the masking is essentially given by a backward cone with an aperture of $30^{\circ}$ and $20^{\circ}$, for the IR and the TV front sections respectively. During the CLDP integration on TORNADO-IDS, analysis was required in order to fully characterise the masking phenomenon and obtain the related mathematical model to be used by the aircraft MC for CLDP inhibition during impingement.

\subsubsection{System error budget}

As an example, a LGB which can achieve a 10 feet Circular Error Probability (CEP) is considered. In this case, it is appropriate that the designator aiming capability must equal or exceed that requirement in order to meet suitable weapon impact criteria. If a hardened shelter access cover, roughly 20 feet in diameter, is considered as a target in our example, this target dimension equates to a 10 feet CEP where $50 \%$ of our hypothetical weapon releases should fall on the target face. Using the tactic shown in Fig. 30 against a vertical target, and choosing a desired release range $\left(R_{R}\right)$ of 35,000 feet, it is necessary that our designator must be capable of keeping its beam on a 20 feet diameter target at a
Terminal Slant Range (TSR) of 70,000 feet. This equates to a total allowable Maximum Error Budget $\left(E B_{\max }\right)$ of $285 \mu \mathrm{rad}$ (20ft/70Kft). The assumption is that the target is designated at the corresponding terminal designation angle $(\psi)$ of $60^{\circ}$ off of the line normal to the target face. This $60^{\circ}$ offset reduces the gross error budget to approximately $143 \mu \mathrm{rad}\left(E B_{\max } \times \cos 60^{\circ}\right)$. This means that all pointing and beam divergence error sources, when added in a worst case fashion, must fall within a cone that subtends $143 \mu \mathrm{rad}$ if $50 \%$ of our hypothetical weapons are to hit the 20 feet target mentioned above. In the light of the above considerations, the maximum allowable error budget can be expressed as:

$$
E B_{\text {max }}=\frac{T_{S} \cdot \cos \psi}{T S R}
$$

where $T_{S}$ is the target size and TSR is the Terminal Slant Range. Using Tactic 2, the terminal slant range can be expressed as:

$$
T S R=\frac{R_{R}}{\cos \psi}
$$

\section{Airborne Lasers Eye-Safety Analysis}

The operation of non-eye safe airborne laser systems for training and test purposes require determination of the hazards associated with their use. In order to determine and evaluate the vulnerabilities, a number of factors that affect eye-safety either directly or indirectly including the geometry of the attack, the altitude profile of the area around the target, the probabilities of inadvertent laser activation and the presence of reflecting materials in the area illuminated by the laser beam have to be taken into account. Unlike ordinary light, intense pulsed light, which are of high-power, can cause biological damage. The laser beam can result in intense ionization effects within the eye tissue and may generate plasma, which subsequently damages the tissues. This damage is due to the associated thermo-mechanical effects. Generally, CW lasers are less injurious than the pulsed lasers for a given total energy. The extent of such injury is dependent on the fact that there arises additional difficulty for the eye in conducting the absorbed heat in the very short period of time that the pulse lasts [175]. The greatest hazard of laser radiation is when the operating region is in the visible and infrared-A bands. It passes through the apparatus of the eye with very little absorption and is then focused with a very high degree of convergence to a very small spot onto the retina, and can result in permanent damage. The actual spot size depends upon the range at which the eye is focussed, but could be as small as 10 $\mu \mathrm{m}$. The effect is particularly severe if the eye is relaxed in which case the laser beam is focussed onto the fovea of the retina and causes loss of both chromatic and detailed vision.

\subsection{Laser safety standards}

The methodology to be used in laser safety assessments is prescribed by various national and international laser safety standards [126-140]. They provide generic suggestions on how to apply the various proposed safety area calculation routines in the case of highly dynamic platforms, such as airborne designation systems.

\subsection{Ocular hazard distance}

According to NATO STANAG 3606 and the Italian SMD-W-001 military laser safety standard (developed in accordance with the STANAG 3606 and quite similar to the JSP 390 British military standard), the Ocular Hazard Distance (OHD) is required for calculating all laser hazard areas [175]. The factors affecting the 
This is the author pre-publication version. This paper does not include the changes arising from the revision, formatting and publishing process. The final paper that should be used for referencing is:

R. Sabatini, M. A. Richardson, A. Gardi, S. Ramasamy, "Airborne laser sensors and integrated systems", Progress in Aerospace Sciences (2015), http: //dx.doi.org/10.1016/j.paerosci.2015.07.002i.

OHD are: design characteristics of the laser system; atmospheric attenuation; atmospheric scintillation; use of laser protective eyewear; viewing through magnifying optics; near-field effects; use of beam attenuating filters. The key system-related parameters to be taken into account for calculation of the OHD are the Maximum Permissible Exposure (MPE) and the Nominal Ocular Hazard Distance (NOHD). The MPE, generally expressed in $\mathrm{J} / \mathrm{cm}^{2}$ or $\mathrm{J} / \mathrm{m}^{2}$, is a function of the Exposure Time $\left(T_{E}\right)$. For example, considering a point laser source with a wavelength of $1064 \mathrm{~nm}$ and a pulse length of $20 \mathrm{nsec}$, the MPE for a single pulse obtained from the JSP 390 standard, is $5 \times 10^{-2} \mathrm{~J} / \mathrm{m}^{2}$. Knowing the MPE for a single pulse, the MPE for a train of pulses can be calculated as follows [154]:

$$
M P E_{T}=M P E_{P} \times \frac{1}{\sqrt[4]{f \times T_{E}}}
$$

where $M P E_{P}$ is the maximum permissible exposure (single pulse), $M P E_{T}$ is the maximum permissible exposure (train), $f$ is the pulse repetition frequency and $T_{E}$ is the time of exposure. There are various expressions used to calculate the value of the NOHD, depending on the characteristics of the laser (i.e., pulsed/CW, single-pulse/train of pulses, Gaussian or non-Gaussian beam, etc.), and the location of the observer (direct illumination or diffuse reflection). NOHD for direct vision of pulsed lasers with Gaussian beam distributions is given by:

$$
N O H D=\frac{\sqrt{\frac{1.27 \times Q \times \sqrt[4]{f \times T_{E}}}{M P E_{P}}-a}}{\Phi}
$$

where $Q$ is the output laser pulse peak energy and $a$ is the output beam diameter. According to the Italian Safety Standard SMDW-001, for non-Gaussian beams, $Q$ should be multiplied by a factor of 2.5. The cumulative OHD arises from the full or partial application of correction factors to the NOHD allowing for nearfield effects, magnifying optics, atmospheric extinction, atmospheric scintillation, beam attenuating filters and protective laser eyewear. If the laser radiation is viewed through magnifying optical instruments, the NOHD will increase to a distance called the Extended Ocular Hazard Distance (EOHD), which can be calculated using the following equation:

$$
E O H D=N O H D \cdot \sqrt{K}
$$

where $K$ is a factor depending on the laser wavelength and the viewing conditions. The calculation of $K$ is given in SMD-W-001 and JSP 390. Both standards include the following equation for calculating the reduction of the hazard distance due to atmospheric attenuation:

$$
O H D_{v}=\frac{N O H D}{2-e^{-0.5 v . N O H D}}
$$

where $v$ is the atmospheric attenuation coefficient. Both standards also refer to the following model for calculating the atmospheric extinction coefficient for laser wavelengths between 400 and $2000 \mathrm{~nm}$ :

$$
v=10^{-3} \cdot \frac{3.91}{V} \cdot{\frac{555^{A}}{\lambda}}^{A}
$$

where $V$ is the meteorological range $(\mathrm{km}), \lambda$ is the laser wavelength (nm) and $A$ is the exponent varying with $V$ and given by $0.585 . V^{0.33}$. It is obvious that, using only eq. (63) for calculating the atmospheric extinction coefficient would lead to underestimated $v$ values for most weather conditions and at most wavelengths of practical interest, which is acceptable for eyesafety calculations but implies a rather conservative simplifying assumption (i.e., absorption is neglected). Together with attenuation, when a laser beam propagates in the atmosphere (especially with slant paths close to the ground) its radiance may be modified by focusing (scintillation) or defocusing effects caused by turbulence. In the first case (scintillation), the values of the beam irradiance may be significantly greater than the MPE, and therefore it is prudent to make some allowance for this effect. According to the SMD-W-001 safety standard, when scintillation is likely to occur at the range (e.g., due to high measured or predicted $C_{n}$ values), NOHD should be modified as follows:

$$
O H D_{S}=2.662 . N O H D
$$

A better approach to this problem is presented in the British safety standard. If $N_{l}$ is less than the parameter $N_{\max }$, where:

$$
N_{\text {max }}=2.2 \times 10^{-7} \cdot \frac{\lambda^{0.64}}{C_{n}{ }^{1.09}}
$$

Then, to take account of scintillation $N_{l}$ is modified to obtain OHDS using the following equation:

$$
O H D_{S}=2.66^{\frac{N_{1}}{N_{\max }}} \cdot N_{1}
$$

where $N_{1}$ is either the NOHD or the cumulative OHD arising from the full or partial application of factors allowing for nearfield effects, magnifying optics, beam attenuating filters, atmospheric extinction and laser protective eyewear. If $N_{1} \geq$ $N_{\text {max }}$, or if it is not possible to determine $C_{n}$ then $O H D_{S}$ is given by:

$$
O H D_{S}=2.66 \cdot N_{1}
$$

Both the British and Italian safety standards present the following equations for correcting the $N_{l}$ parameter (i.e., the NOHD or the cumulative OHD calculated taking into account a part or all other correction factors) due to laser protective eyewear $\left(\mathrm{OHD}_{\mathrm{PE}}\right)$, nearfiled effects $\left(\mathrm{OHD}_{\mathrm{NF}}\right)$, and beam attenuating filters $\left(\mathrm{OHD}_{\mathrm{AF}}\right)$ :

$$
\begin{gathered}
O H D_{P E}=N_{1} \cdot 10^{-O D / 2} \\
O H D_{N F}=N_{1} \cdot \sqrt{1-\left(\frac{R_{N}}{N_{1}}\right)^{2}} \\
O H D_{A F}=N_{1} \cdot \sqrt{\tau}
\end{gathered}
$$

where OD is the eyewear optical density, $\mathrm{RN}$ is the 'near-field' range of the laser and $\tau$ is the transmittance of the beam attenuating filter at the laser wavelength.

\subsection{Hazard area, buffer zone and extended buffer zone}

The laser hazard areas are defined and modelled and in particular, the following areas are identified:

- ALS Beam Hazard Area (A-BHA), defined as the area which may be illuminated by the laser beam in the event of inadvertent firing;

- $\quad A L S$ Buffer Zone (A-BZ), given by the sum of the area directly illuminated by the laser beam during the firing (a function of beam output diameter and divergence) and the area around the laser beam that may be inadvertently illuminated considering the overall pointing accuracy of the LTD, the reaction time of the aircrew and the probability of failure of the system;

- ALS Extended Buffer Zone (A-EBZ), defined as the area which may be illuminated due to specular reflection within the A-BZ. The existence of an EBZ can be prevented by removing all possible reflectors lying within the BZ (e.g., residues of previous bomb drops, metal objects, etc.). 
This is the author pre-publication version. This paper does not include the changes arising from the revision, formatting and publishing process. The final paper that should be used for referencing is:

R. Sabatini, M. A. Richardson, A. Gardi, S. Ramasamy, "Airborne laser sensors and integrated systems", Progress in Aerospace Sciences (2015), http: //dx.doi.org/10.1016/j.paerosci.2015.07.002i.

For air-to-ground LTD operations, the A-BHA is given by the intersection with the ground of a sphere with centre at the aircraft location in space and a radius equivalent to the OHD (Fig. 30). Therefore, the radius of the A-BHA $\left(R_{B H A}\right)$ is given by the following equation:

$$
R_{B H A}=\sqrt{O H D^{2}-Z^{2}}
$$

where $z$ is the AGL aircraft altitude. From the definition given above, it appears evident that, in the practical case of an airborne LTD (A-LTD), the actual existence of an A-BHA is related with the following factors:

- inadvertent activation of the laser in the various modes of the LTD;

- inadvertent rotation of the LOS during commanded laser activation.

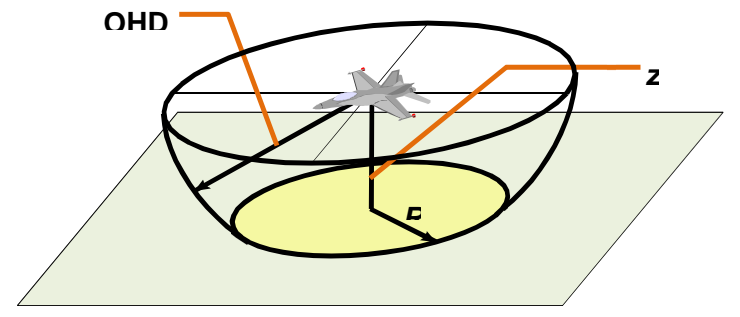

Fig. 30. ALS Beam Hazard Area (A-BHA) geometry.

Therefore, it is acceptable to calculate the A-BHA using the OHD for exposition to a single pulse. As an example, we consider the probabilities given in Table 8 for a typical airborne A-LTD system (entire system operational life).

\section{Table 8}

Hazard probabilities in the various A-LDT modes.

\begin{tabular}{|c|c|c|}
\hline A-LTD Mode & Hazardous Event & Probability \\
\hline OFF & Inadvertent activation & 0 \\
\hline ON & Inadvertent activation & $5 \mathrm{E}-16$ \\
\hline SLAVE/TRACK & Inadvertent activation & $8 \mathrm{E}-9$ \\
\hline Laser Arm & Inadvertent activation & $3 \mathrm{E}-4$ \\
\hline $\begin{array}{c}\text { Laser Fire } \\
\text { (SLAVE/TRACK) }\end{array}$ & $\begin{array}{c}\text { Inadvertent LOS } \\
\text { rotation }\end{array}$ & $2 \mathrm{E}-6$ \\
\hline
\end{tabular}

The data in Table 8 are generally linked to the reliability data of the aircraft avionics systems connected with the LTD. In our example, we assume that the A-LTD system is commanded by the Weapon Systems Operator (WSO) through a cockpit control panel with a SAFE and an ARM position (i.e., a stand-by mode in which the laser cavity is powered but the laser beam is not emitted), and that the laser emission (laser FIRE mode) is commanded by the WSO with a dedicated hand control (also used for manual target tracking). In this case, the probability of inadvertent laser activation $\left(P_{S A F E \rightarrow F I R E}\right)$ is given by:

$$
P_{S A F E \rightarrow F I R E}=P_{S A F E \rightarrow A R M} \times P_{A R M \rightarrow F I R E}
$$

Assuming that the probability of inadvertent activation of the ARM mode from the SAFE condition $\left(P_{S A F E \rightarrow A R M}\right)$ referred to the entire A-LTD operational life is 7E-4, and that the probability of inadvertent activation of the FIRE mode from the ARM condition $\left(P_{A R M \rightarrow F I R E}\right)$ is 1 for missions in which the WSO acts on the cockpit commands (i.e., simulated or real attack missions) and $1 \mathrm{E}-2$ in missions were the WSO does not act on the cockpit commands (e.g., ferry flights), then the overall probabilities of inadvertent laser activation (with the A-LTD in SAFE mode) are given in Table 9.

Table 9

A-LTD risk levels with laser SAFE.

\begin{tabular}{|c|c|c|}
\hline A-LTD Mode & Mission & P $_{\text {SAFE } \rightarrow \text { FIRE }}$ \\
\hline ON/SAFE & Ferry flight & $7 \mathrm{E}-6$ \\
\hline ON/SAFE & Test/Training & $7 \mathrm{E}-4$ \\
\hline
\end{tabular}

The A-BZ is given by the sum of the area directly illuminated by the laser beam during the firing (a function of beam output diameter and divergence) and the area around the laser beam that may be inadvertently illuminated considering the overall pointing accuracy of the LTD system, the reaction time of the aircrew and the probability of failure of the system. In other terms, at any instant, the A-BZ shape can be approximated by an ellipse where the target occupies one of the foci. With reference to Fig. 31, the dimensions of the A-BZ can be calculated for any given location of the aircraft in space by using the following equations:

$$
R_{1}=\left[\frac{1}{\tan \varphi}-\frac{1}{\tan (\varphi+\delta)}\right] . Z
$$

$$
R_{2}=\frac{Z \cdot \sin \delta}{\sin \varphi \cos \delta}
$$

$$
R_{3}=\left[\frac{1}{\tan (\varphi-\delta / 2)}-\frac{1}{\tan \varphi}\right] \cdot Z
$$

where $\varphi$ is the angle between LOS and horizontal in the plane containing the LOS and $\delta$ is the sum of pointing error and safety margin.

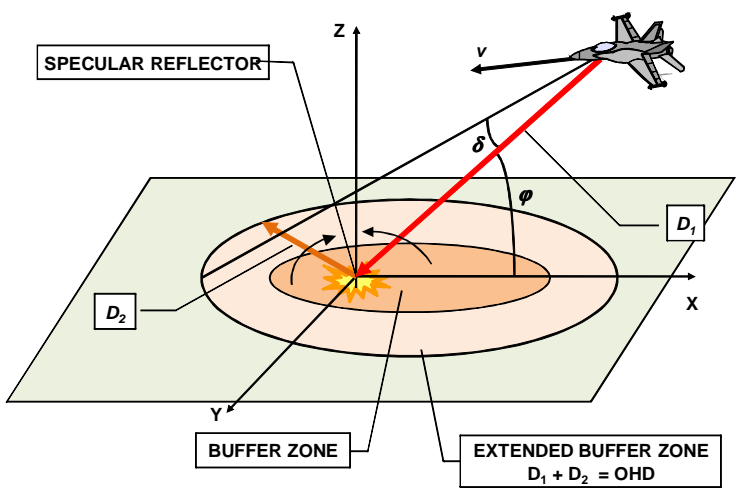

Fig. 31. ALS Buffer Zone (A-BZ) geometry.

The last area to be determined is the ALS Extended Buffer Zone (A-EBZ), defined as the area which may be illuminated due to specular reflection within the A-BZ. The existence of an A-EBZ can be prevented by removing all possible reflecting surfaces 
This is the author pre-publication version. This paper does not include the changes arising from the revision, formatting and publishing process. The final paper that should be used for referencing is:

R. Sabatini, M. A. Richardson, A. Gardi, S. Ramasamy, "Airborne laser sensors and integrated systems", Progress in Aerospace Sciences (2015), http: //dx.doi.org/10.1016/j.paerosci.2015.07.002i.

within the A-BZ (e.g., residues of previous bomb drops, metal objects, etc.). However, while evacuation of people can be performed quite easily, removal of all reflecting materials from the A-BZ can be a very demanding task for a test range and it is often impracticable. Therefore, in general, we must consider the A-EBZ as the laser hazard area to be evacuated. Determination of the A-EBZ area is not an easy task, since its dimension and shape are dependent upon the aircraft position in space and its angular velocity with respect to the reflection points located in the A-BZ (varying continuously during a mission). This is true because the hazard to the naked human eye is a function of the exposure time $\left(T_{E}\right)$ and $T_{E}$ to a specifically reflected laser beam varies with aircraft relative velocity. It is therefore necessary to implement a simulation tool in order to calculate the aircraft envelope limitations due to a certain pre-defined maximum evacuation area or, conversely, the dimension of the evacuation area required with a certain pre-defined mission profile. Fig. 32 exemplifies the various geometric and physical parameters involved.

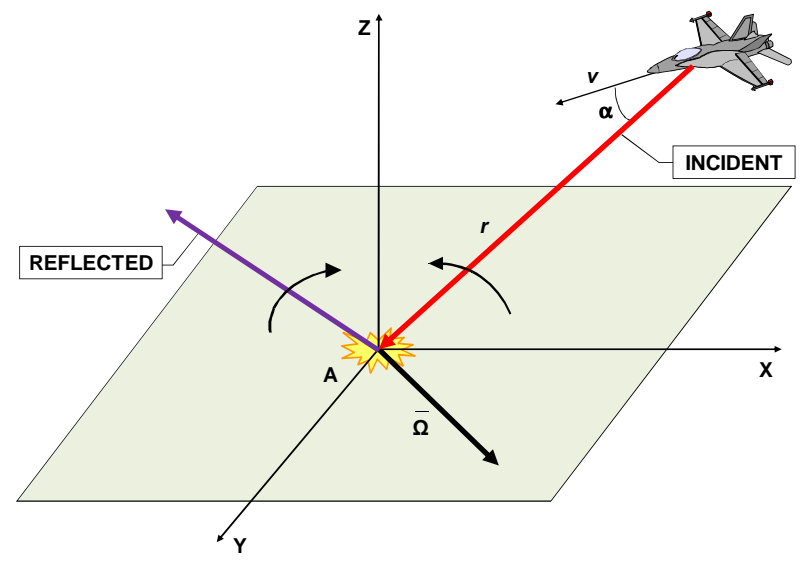

Fig. 32. ALS Extended Buffer Zone (A-EBZ) geometry.

\subsection{Safety verification algorithm}

In a Cartesian reference frame centred at the point of intersection of the LOS with the ground (point $A$ in Fig. 30), the velocity vector of the aircraft $(\bar{v})$ can be expressed as:

$$
\bar{v}=\bar{\Omega} \times \bar{r}
$$

where $\bar{\Omega}$ is the aircraft angular velocity vector and $\overline{\mathrm{r}}$ is the aircraft position vector. Therefore, the module of the angular velocity of the laser beam with respect to the reflection point on the ground, is given by:

$$
|\bar{\Omega}|=\frac{|\bar{v}|}{|\bar{r}|} \times \sin \alpha
$$

where $\alpha$ is the angle between the aircraft position and the velocity vector. This is given by:

$$
\alpha=\arccos \frac{\bar{v} \cdot \bar{r}}{|\bar{v}| \cdot|\bar{r}|}
$$

Therefore, knowing the vectors $\bar{v}$ and $\bar{r}$ at any point in space it is possible to calculate the corresponding value of $|\bar{\Omega}|$. This value can then be compared with the minimum sweep velocity admitted for the reflected laser beam, which is a function, at any point on the ground, of beam dimension (i.e., output area and divergence) and maximum permissible exposure time $T_{E(M A X)}$.

In other terms, since the actual exposure time of an observer to the reflected laser radiation is a function of the angular velocity $\bar{\Omega}$, of the beam divergence and of the distance between the observer and the point A, knowing the effective time of exposure (and therefore the effective OHD), it is possible to verify the safety of a scenario, taking into account the elements including aircraft position and velocity, observer position, reflection point and laser characteristics, and comparing the effective NOHD with the sum of the distances observer-point A and point A-aircraft. The aforementioned procedure is illustrated in Fig. 33.

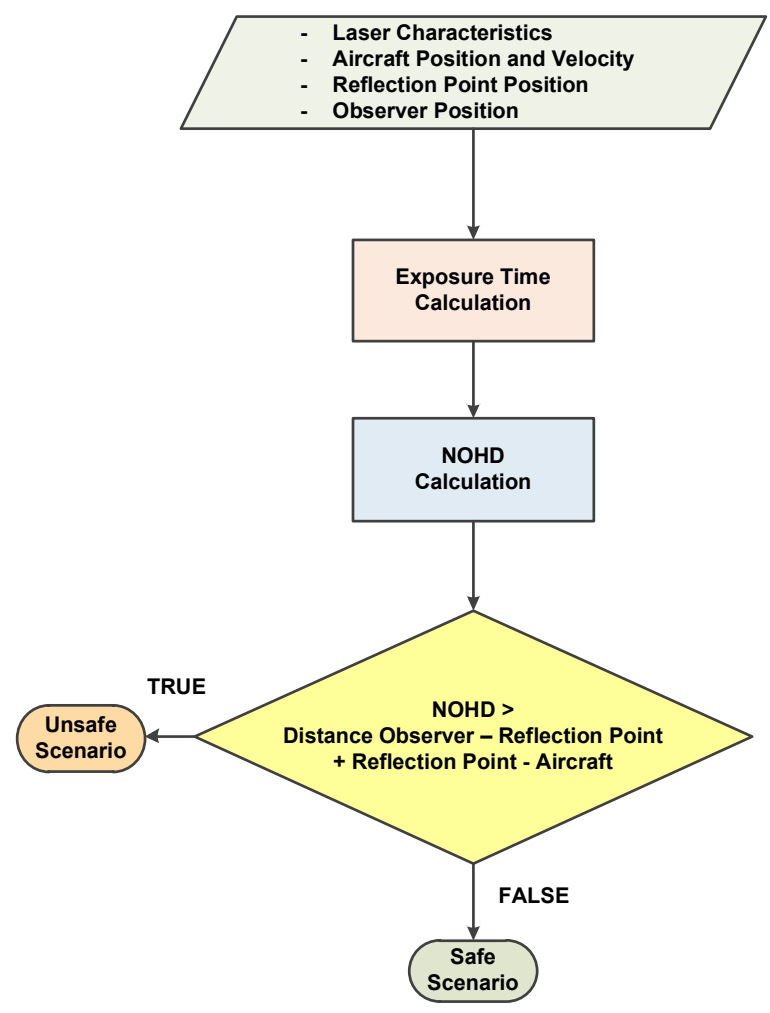

Fig. 33. ALS safety verification algorithm.

Knowing the dimensions of the BZ, it is possible to verify the observer's safety, using the procedure described in Fig. 35 in an iterative manner for the entire BZ area. Therefore, simulation is required in order to determine the flight envelope restrictions due to eye-safety (or, conversely, the ground evacuation requirements for a given aircraft flight profile).

\subsection{Test and training safety procedures}

In order to guarantee a safe and practical employment of ground laser systems, the results obtained from laser hazard areas calculations are used to define appropriate procedures, which are then implemented at the laser range. These procedures are drafted by taking into account the national safety regulations and standards. Due to the very high energy output and low divergence of the ground laser systems, in many real cases, the calculated laser hazard areas for the required mission geometries are not compatible with the range size. Hence, in certain cases, additional criteria have to be adopted in order to allow a safe execution of test/training tasks with representative mission geometries and cooperative scenarios. The safety margin adopted for Ground Laser Target Designators (GLS) Buffer Zone (G-BZ) calculations is defined by the applicable laser safety standards. Based on the standards defined, the SM to be adopted for ground systems is 10 , 5 or $2 \mathrm{mrad}$ depending on the stability of the system LOS. Procedures have been developed in accordance with the GLS 
This is the author pre-publication version. This paper does not include the changes arising from the revision, formatting and publishing process. The final paper that should be used for referencing is:

R. Sabatini, M. A. Richardson, A. Gardi, S. Ramasamy, "Airborne laser sensors and integrated systems", Progress in Aerospace Sciences (2015), http: //dx.doi.org/10.1016/j.paerosci.2015.07.002i.

safety analysis concepts and with the safety standards recommendations including:

- A target should be used with shape and dimensions adequate to contain the entire elliptical footprint of the G-BZ at the defined GLS-target slant-ranges and GLS LOS incidence angles.

- The G-BZ has to be calculated taking into account the output diameter of the laser beam (a), the beam divergence $(\Phi)$, the Pointing Error (PE) of the GLS, and the additional Safety Margin (SM) contemplated by the applicable safety standards.

- The target has to be free from crevices or apertures and to be characterised by a diffuse reflectivity.

- The intersection of the G-BZ with the ground and natural/man-made obstacles should be avoided.

- The GLS operator should verify, before activating the laser, that the LOS of the GLS is aimed at the centre of the selected target.

- If it is not possible to use a target with the required characteristics, all reflecting objects present in the GEA should be removed and flying into the HAS without suitable aircrew protection is prohibited.

- If it is not possible to remove reflecting objects in the GEA, flying into the EHAS without suitable aircrew protection is prohibited.

- Access to the G-BZ should be prohibited to unprotected personnel.

- The use of magnifying optical instruments not suitably filtered should be prohibited in the entire laser range.

\subsection{Operational considerations}

There are important operational considerations regarding the test and training procedures. The procedures involving the removal of reflecting objects or restricting flight into the EHAS are not commonly implemented. The removal of reflecting objects in the GEA is in fact challenging. If it is not possible to remove reflecting objects in the GEA, it may easily lead to a condition wherein the EGEA dimensions exceeds the laser range perimeter. Furthermore, implementation of such procedures may require creation of dedicated no-fly zones (associated with HAS and EHAS respectively) which may exceed the dimensions of the range airspace. The selection of the best procedure matching both the eye-safety requirements and the need of executing test/training missions depends on a variety of conditions including GLS-target geometry, terrain profiles, co-operative tasks and guided weapons deliveries representative of the real operational scenarios. Safety considerations for High-Intensity Lights (HIL) directed into the airspace have to be considered to mitigate any adverse implications on safety and health [176-178]. Laser safety considerations with respect to atmospheric scintillations are also taken into account to evaluate the operational criteria [179-182].

\section{Airborne Laser Systems Test and Evaluation}

A number of experimental activities have to be performed on airborne laser systems and these typically include laboratory, ground and flight testing. The range of possible experimental requirements is very wide and typical examples include:
- Laser Beam Profiling (LBP);

- Surface reflectance;

- Angular resolution;

- Sensor bandwidth;

- Sensor field-of-view;

- Sensor detection threshold;

- Boresight and Pointing accuracy (static and dynamic);

- Sensor calibration;

- Frame-rate optimisation;

- Protection filter and eye-wear verification;

- Spot quality;

- Effects of atmospheric extinction.

Additionally, the development of avionics software for airborne laser systems typically requires moving into the following formal verification and validation process:

- Stage A Testing, involving verification of stand-alone software routines by using mathematical models to stimulate/emulate avionics functions;

- Stage B Testing, where combined algorithms are tested involving single or group of avionics equipment (real or simulated). Mathematical models are used to stimulate software for interaction with equipment firmware to check the compatibility and reaction of the involved sub-systems.

- Stage C Testing, where fully representative rigs of the aircraft avionics system are used to evaluate performance and reliability of software in closed loop testing. Software confidence level ascertained during Stage $\mathrm{C}$ trials permit to authorize testing in flight (i.e., identification of airworthiness flight limitations as applicable).

- Stage D Testing, in which flight test activity are undertaken to verify software reliability and operational effectiveness, and to define operational limitations.

The software development process is schematically illustrated in Fig. 34. 
This is the author pre-publication version. This paper does not include the changes arising from the revision, formatting and publishing process. The final paper that should be used for referencing is:

R. Sabatini, M. A. Richardson, A. Gardi, S. Ramasamy, "Airborne laser sensors and integrated systems", Progress in Aerospace Sciences (2015), http: //dx.doi.org/10.1016/j.paerosci.2015.07.002i.

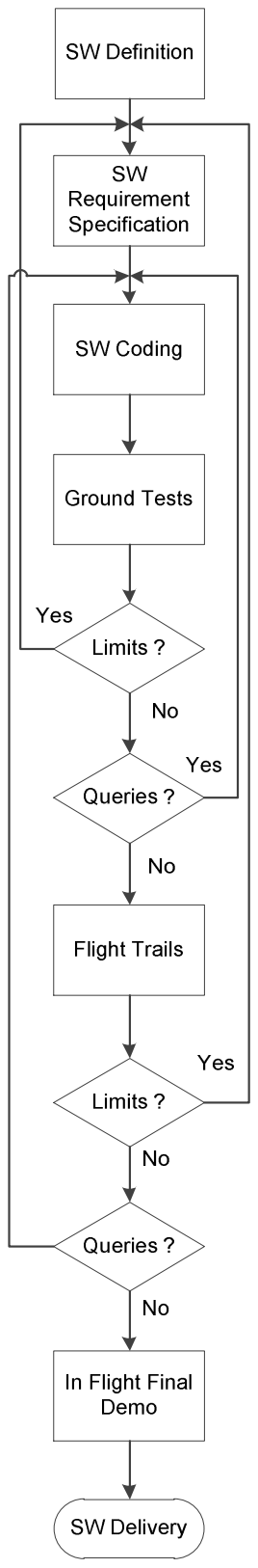

Fig. 34. Avionics software development process.

Based on extensive experience with airborne laser systems, interaction among the different simulation and analysis processes highlights the need to carry out flight test activity in order to:

- Verify software integrity, especially in those critical areas where rigs and simulation testing are were not the appropriate vehicles;

- Evaluate sub-system operability in a real environment;

- Suggest further development areas or optimisation of existing functions to improve reliability;

- Assess man/machine interface and workload.

\subsection{Modelling and Simulation for Systems Testing}

Modelling and simulation activities in support of system testing typically include:
- System performance prediction;

- Verification of eye-safety requirements;

- Mission planning and effectiveness evaluation.

\subsubsection{Performance Prediction}

The range performances of a particular emitter/sensor combination in specific conditions can be estimated using specifically developed software, which implement the models described in section 2. The input data for detailed performance estimation include the following parameters:

- Beam diameter at the source;

- Beam divergence at the source;

- Beam polarisation;

- Wavelength;

- Peak energy of the emitter;

- Pulse duration;

- Pulse Repetition Frequency (PRF);

- Receiver Field of View (FOV);

- Receiver Minimum Detectable Power Density (MDPD);

- Target reflectance, ideally also in terms of Bidirectional Reflectance Distribution Function (BRDF);

- Relative Humidity (RH);

- Temperature;

- Visibility;

- Wind speed (average and standard deviation);

- Atmospheric composition;

- Atmospheric precipitation (type and amount).

The range emitter-target can be calculated as a function of the range sensor-target and a family of curves can be traced for different orientations of the target over the horizon (i.e., different values of the angles $Q_{t}(M A X)$ and $\left.Q_{r}(M A X)\right)$. Using the curves it is possible to determine whether or not the experiment/activity can be performed with a certain estimated minimum illumination time. Given the platform initial conditions (i.e., velocity and trajectory) before designation is initiated, it is possible to estimate the designation time, taking into account the time required by an LGW from these initial conditions to stabilise towards the target. If the guidance algorithms are unknown, it is possible to roughly estimate the designation time by assuming a straight trajectory of the LGW towards the target and a velocity in the final portion of its drop correspondent to the maximum theoretical velocity of the weapon. With these assumptions, the minimum theoretical range LTD-target before designation can be plotted in the graphs and consequently the maximum range of the aircraft at the beginning of the designation is identified. Fig. 35 represents the performance calculated for a specific A-LTD/LGB combination, with different values of visibility $(\mathrm{V})$ in the absence of rain. 
This is the author pre-publication version. This paper does not include the changes arising from the revision, formatting and publishing process. The final paper that should be used for referencing is:

R. Sabatini, M. A. Richardson, A. Gardi, S. Ramasamy, "Airborne laser sensors and integrated systems", Progress in Aerospace Sciences (2015), http: //dx.doi.org/10.1016/j.paerosci.2015.07.002i.
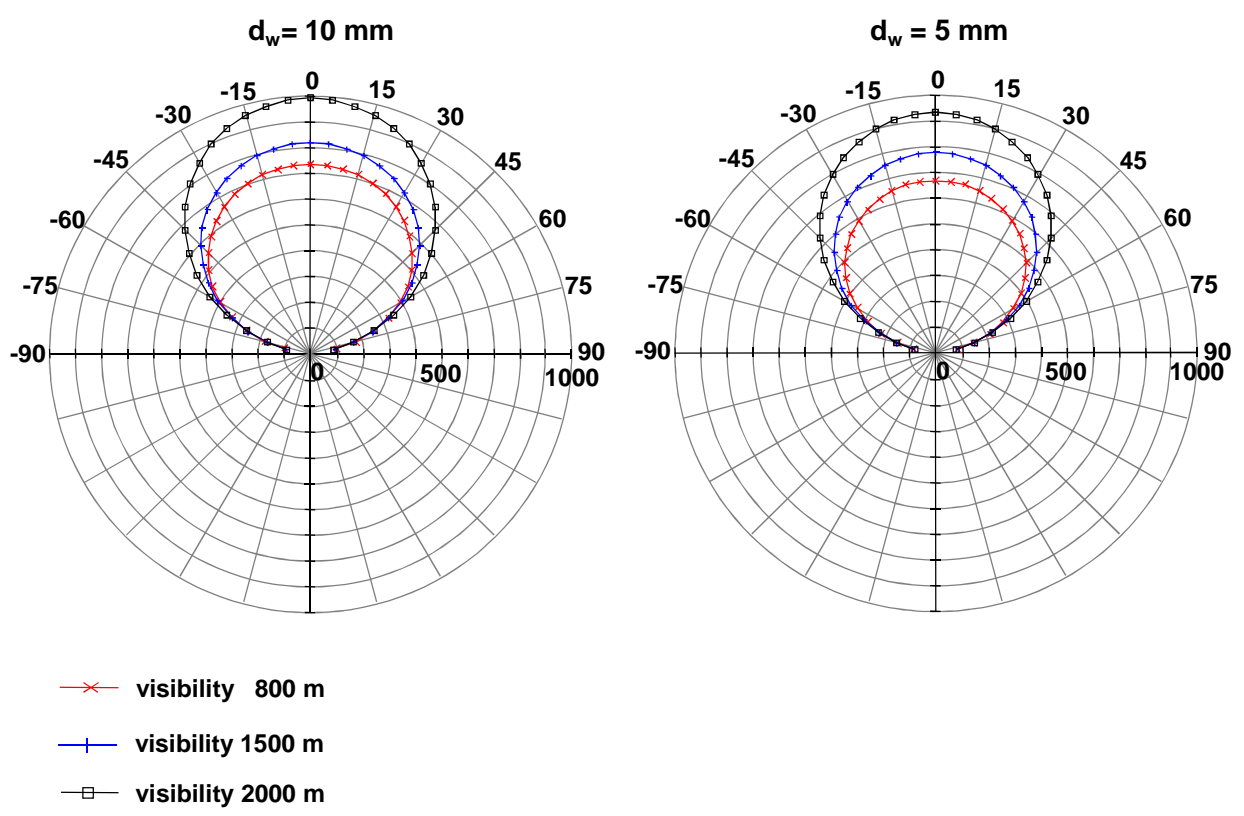

Fig. 35. Results of the performance prediction calculations for different diameters of the target.

\subsubsection{Eye-safety verification}

Simulation tools are developed especially in the areas of eyesafety and system performance analysis and are presented in [178]. Theoretical and empirical models described earlier can be implemented in software modules, allowing a comprehensive assessment of test/training laser mission constraints and operational feasibility, as well as post-mission data analysis. The typical assumptions adopted for implementing eye safety verification for both ALS and GLS systems are:

- The reflecting surface (BZ) is perfectly planar; this assumption is conservative in the case of convex surfaces laying within the BZ (these would in fact determine an increase of divergence with consequent reduction of the energy density at the observer location); while, in the case of concave surfaces, it is extremely improbable that their presence in the BZ can determine focusing (reduction of divergence) of the laser beam.

- The laser beam reflection is totally specular; this assumption is conservative from a safety point of view, because the energy density of a specularly reflected laser beam is always greater than in the real case.

- For the A-SVP the entire A-BZ is considered as a specular reflector; this is a conservative assumption for eye-safety calculations. Moreover, considering the entire BZ as the actual reflector, the presence of any reflecting material inside the BZ is not relevant for safety (i.e., objects removal is not required within the $\mathrm{BZ}$, but only evacuation of the ground personnel).

- For the G-SVP the target surface is considered as a diffuse reflector; this is a condition that is imposed when designing the laser target test range.

- Atmospheric attenuation of the laser beam is not considered; this assumption implies that the entire energy emitted by the LTD reaches the observer location. This is a conservative assumption, since atmospheric absorption and scattering effects are neglected.
- The NOHD is calculated for direct vision of a Gaussian laser beam; the assumption of direct vision is conservative, since the observer is assumed to look directly at the laser source, instead of a reflected beam; while a Gaussian distribution of the laser beam is applicable in practice for most ALS.

- Allowance is made for atmospheric scintillation effects; this assumption is conservative as it implies, in the absence of $\mathrm{C}_{\mathrm{n}}$ measurements, that $\mathrm{OHD}_{\mathrm{S}}=2.662$. NOHD.

- For the A-SVP a significant instability of the system LOS (i.e., maximum value experimentally observed) is considered.

The methodology for calculating the probabilities of hazardous events during real missions is detailed in [183]. As an example, the limitations in flight profile due to eye-safety for a typical ALTD system (100 mJ pick energy, $10 \mathrm{~Hz}$ PRF and $80 \mathrm{nsec}$ pulse duration, $0.2 \mathrm{mrad}$ divergence and $60 \mathrm{~mm}$ output beam diameter) operating in automatic track mode over a circular range area of 5 $\mathrm{km}$ in radius are shown in Fig. 36. For comparison, the areas for safe positioning of a G-LTD system having similar characteristics to the A-LTD above are shown in Fig. 37.

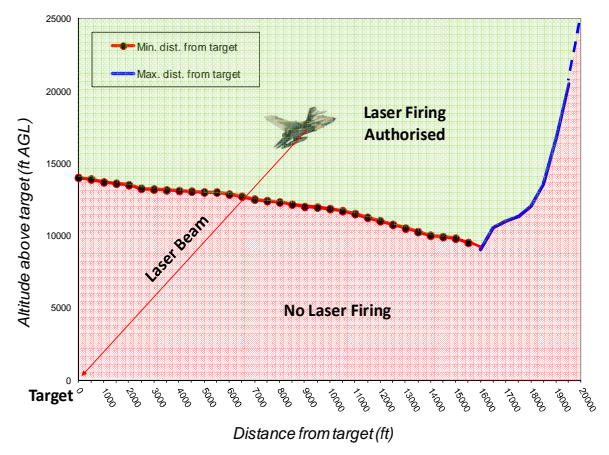

Fig. 36. A-LTD laser safety flight restrictions. 
This is the author pre-publication version. This paper does not include the changes arising from the revision, formatting and publishing process. The final paper that should be used for referencing is:

R. Sabatini, M. A. Richardson, A. Gardi, S. Ramasamy, "Airborne laser sensors and integrated systems", Progress in Aerospace Sciences (2015), http: //dx.doi.org/10.1016/j.paerosci.2015.07.002i.

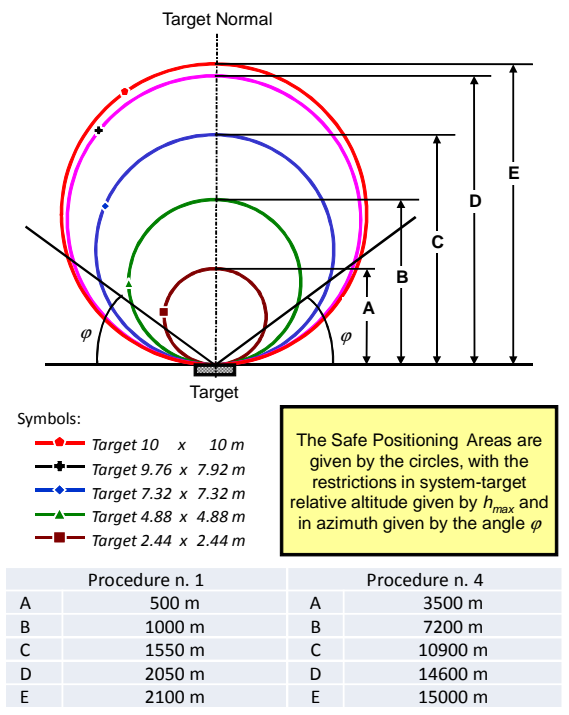

Fig. 37. Calculated areas for safe positioning of a ground-based laser system.

\subsubsection{Mission planning and effectiveness evaluation}

Accurate mission planning and post-mission effectiveness evaluation require appropriate knowledge of target signatures (e.g., reflectivity, BRDF/LCS), background characteristics, atmospheric attenuation, hardware performance (e.g., detectors, pointing/tracking and FOV), mission geometry (e.g., masking, laser grazing angle, aircraft and target motion) and, in some cases, human operator performance (e.g., target search and acquisition with TV/FLIR aids, manual laser firing, etc.). Furthermore, for mission planning purposes, it is also important to take into account eye-safety issues (especially for test and training activities with ALS systems). A comprehensive software tool for ALS performance analysis and mission planning was developed in [183]. Fig. 38 shows an example of the associated 3D visualisation formats.
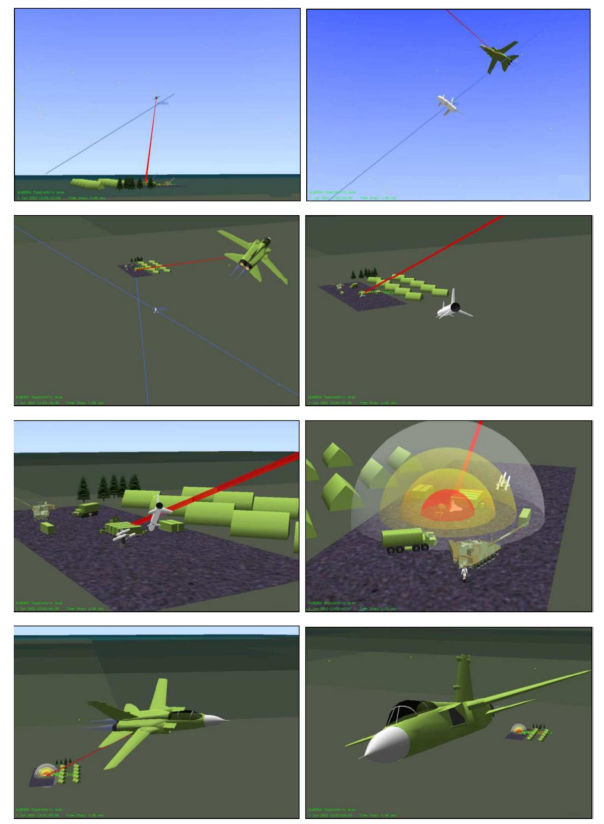

Fig. 38. ALS-MPP 3D simulation O/P-I.

\subsection{Laboratory experimental activities}

\subsubsection{Laser beam profiling}

Laser Beam Profiling (LBP) is performed to examine the output characteristics of a laser emitter before performing further experimental activities. In particular, some experiments mandate well-defined and repeatable conditions, which require accurate definitions of the validity and applicability of the test and these requires LBP. The features common to most commercial laser beam profilers include:

- Intensity distribution analysis, also determining beam width, spatial profile, quality, divergence, jitter and astigmatism;

- Gaussian fit analysis;

- Image, capture, store, and playback of 2D and 3D intensity plots;

- Printing of text and pictures.

The main technologies available for laser beam diagnostics are:

- Direct illumination camera;

- Moving mechanical slit or knife edges to scan across the incoming beam.

The main advantage of mechanical scanning devices over camera type laser beam profilers is the large dynamic range that allows accurate measurements of beams with both high and low intensities. On the other hand, camera type laser beam profilers are excellent for fast and detailed analysis of laser beam intensity profiles, but are limited in their accuracy due to a relatively low dynamic range. However, to overcome the limited dynamic range of a camera type beam profiler and accurately measure faint laser beam structures, it is possible to sample the beam several times, each measurement being performed at a different attenuation or electronic shutter speed. Examples of a LBP results are shown in Fig. 39.
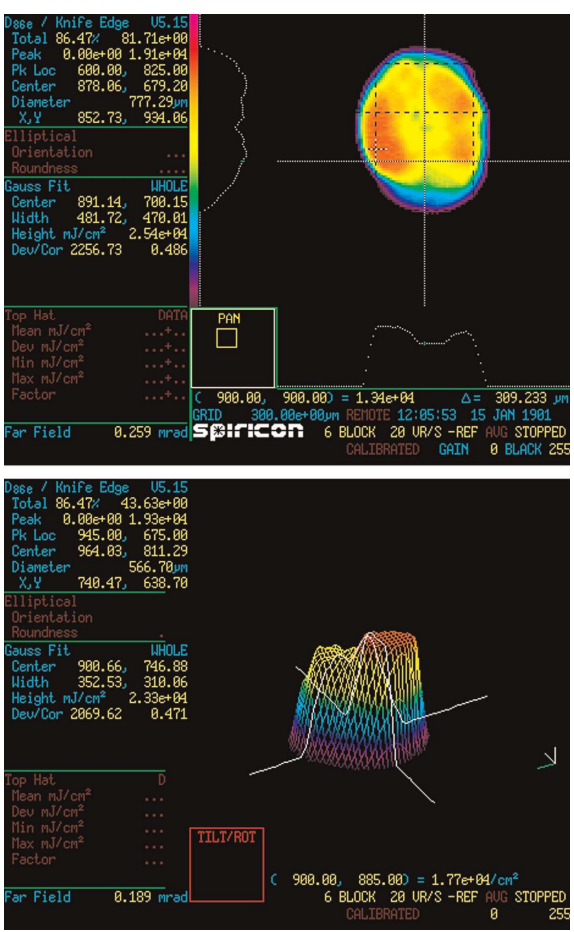
This is the author pre-publication version. This paper does not include the changes arising from the revision, formatting and publishing process. The final paper that should be used for referencing is:

R. Sabatini, M. A. Richardson, A. Gardi, S. Ramasamy, "Airborne laser sensors and integrated systems", Progress in Aerospace Sciences (2015), http: //dx.doi.org/10.1016/j.paerosci.2015.07.002i.

Fig. 39. Spiricon ${ }^{\mathrm{TM}}$ (Ophir Oprtonics Ltd.) $2 \mathrm{D}$ and $3 \mathrm{D}$ display format.

\subsubsection{Surface reflectance measurements}

The knowledge of target surface reflectance properties is essential to accurately predict the performances of ground and airborne laser systems. At laser wavelengths, most material behave as diffuse (Lambertian) reflectors but, for several applications, it is also important to quantify the specular reflection components. A conceptual representation of the diffuse and specular reflection components is shown in Fig. 40 and their variation as a function of grazing angle is given in Fig. 41.

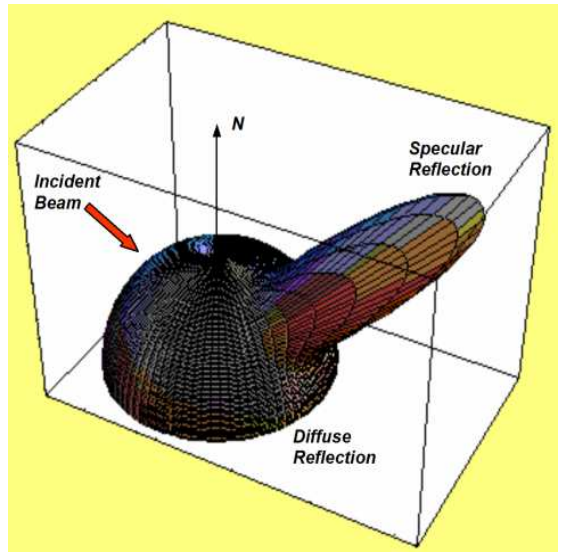

Fig. 40. Diffuse and specular reflectance.

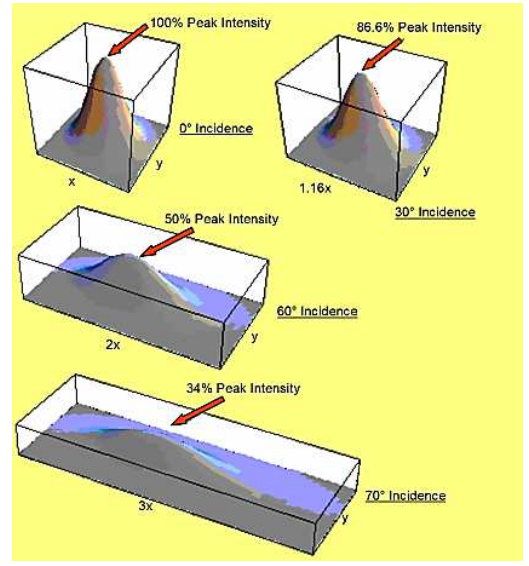

Fig. 41. Diffuse and specular reflectance as a function of the grazing angle.

Laboratory test activities can be performed to determine the reflectance properties of various materials/paints (encountered operationally or in test/training activities) in the visible and infrared portions of the spectrum. This can be both in terms of total integrated reflectance in a certain band, and in terms of reflectance at specific wavelengths and grazing angles. The Bidirectional Reflectance Distribution Function (BRDF) represents the reflectance and its dependencies on the azimuth and zenith angles for both the source and the observer. The BRDF is defined as the ratio of the radiance of a sample to the irradiance upon that sample, for given azimuth and zenith angles of incidence and azimuth and zenith angles of scattering. Fig. 42 depicts the fundamental geometric characteristics involved. The origin of the beam coordinate system is the point at the centre of the irradiance $(I)$ on the sample surface. The $Z B$ axis is normal to the sample surface, and the $X B$ axis lies in the plane defined by $Z B$ and $I$. The incident direction is specified by two angles: the zenith angle $\left(\theta_{i}\right)$, and the azimuth angle $\left(\phi_{i}\right)$, where $\phi_{i}=\pi$ by definition. Similarly, the observer direction is specified by the zenith angle $\left(\theta_{s}\right)$, and the azimuth angle $\left(\phi_{s}\right)$.

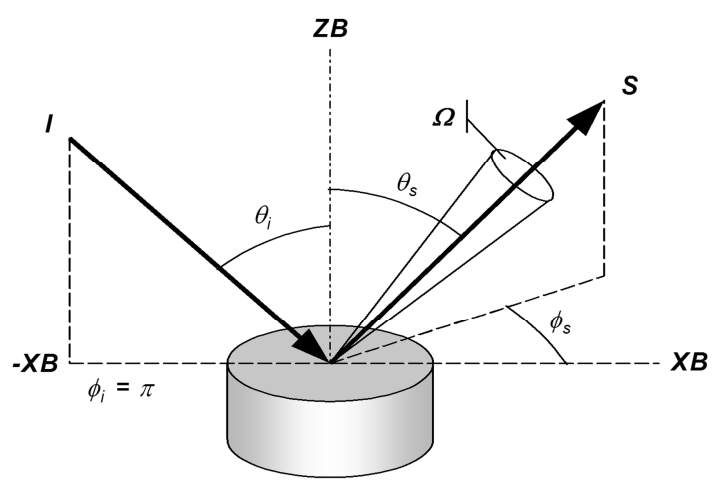

Fig. 42. Geometric characteristics involved in the BRDF.

The instrument providing BRDF measurements is the gonioreflectometer. A typical gonioreflectometer is composed by four main parts: a laser assembly (including the laser emitter and the intensity/polarization control units), a kinematic complex, a detector assembly (including the collimator, the polarizing filter and the electro-optic detector), and a processing unit (including the energy/power meter and a motion control unit). A planar (azimuth rotation) gonioreflectometer is represented in Fig. 43.

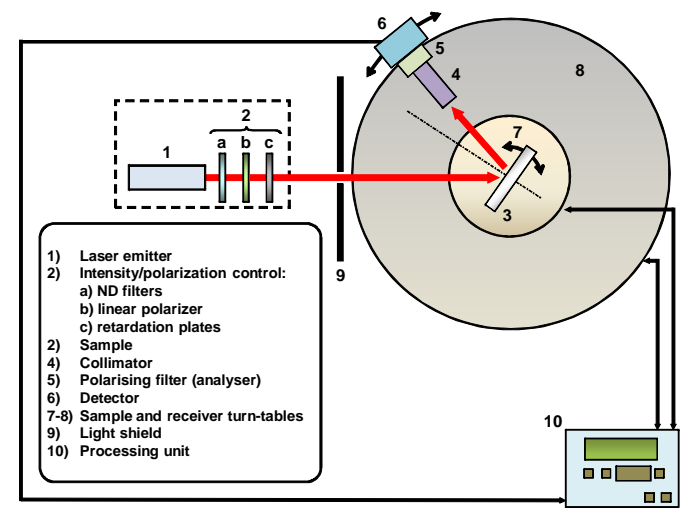

Fig. 43. Planar gonioreflectometer for BRDF measurements.

In order to measure the BRDF, the sample is illuminated by the laser assembly with a collimated beam from a range of incident orientations. The detector assembly, subtending a solid angle $\Omega$ and observing the entire illuminated area, is positioned at every orientation and the radiated energy is measured and transmitted to the processing unit. For any gonioreflectometer configuration, an average sample irradiance $\left(E_{e}\right)$ is calculated from the power $P_{i}$ incident on the sample and the illuminated area $A$. An average sample radiance $L_{e}$ is calculated from the power $P_{s}$ collected by the receiver, the receiver solid-angle, and the area of illumination. Therefore, the sample BRDF is calculated as the ratio of these two quantities:

$$
B R D F=\frac{L_{e}}{E_{e}}=\frac{\left(\frac{P_{s}}{\Omega A \cos \theta_{s}}\right)}{\left(\frac{P_{i}}{A}\right)}=\frac{P_{s}}{P_{i} \Omega \cos \theta_{s}} \quad\left[s r^{-1}\right]
$$


This is the author pre-publication version. This paper does not include the changes arising from the revision, formatting and publishing process. The final paper that should be used for referencing is:

R. Sabatini, M. A. Richardson, A. Gardi, S. Ramasamy, "Airborne laser sensors and integrated systems", Progress in Aerospace Sciences (2015), http: //dx.doi.org/10.1016/j.paerosci.2015.07.002i.

Alternatively, the relative radiance of the sample may be measured versus that of a standard whose BRDF is known for the bi-directional geometry in question. The sample BRDF may then be calculated by multiplying the resulting ratio by the known BRDF of the standard. The BRDF of a given sample is closely related to the bi-directional reflectance factor. This is defined as the ratio of the flux scattered in a given direction by the sample, to that which would be scattered in that direction by the perfect reflecting diffuser, under identical conditions of illumination. The relation between BRDF $(B)$ and bi-directional reflectance factor $(R)$ is expressed by:

$$
R\left(\theta_{i}, \phi_{i}, \theta_{s}, \phi_{s}\right)=\pi B\left(\theta_{i}, \phi_{i}, \theta_{s}, \phi_{s}\right)
$$

It is important to observe that the BRDF of a surface having perfectly diffuse radiance (Lambertian) would be constant for all bi-directional geometries. However, the power collected by the receiver $\left(P_{s}\right)$ is strongly dependent on the irradiance angles $\left(\theta_{s}\right.$ and $\phi_{s}$ ), and becomes very small as $\theta_{s}$ and/or $\phi_{s}$ approach $\pi / 2$. For this reason, the effects of noise and other sources of measurement error typically become much more pronounced at large scatter angles. Both the polarization state of the incident irradiance and the polarization bias of the receiver are important factors in BRDF measurements. Many scattering materials significantly depolarise incident flux, while other materials selectively absorb flux with a certain polarization. Therefore, a comprehensive characterization of the sample scattering properties also requires the evaluation of polarization effects. Laboratory BRDF measurements are therefore performed with various incidence angles (e.g., $\psi=0^{\circ}, 30^{\circ}, 45^{\circ}$ and $60^{\circ}$ ) and both parallel $(P)$ and perpendicular $(S)$ polarization of the incident laser beam. BRDF measurements relative to a diffuse reflector are shown in Fig. 44 (a) and (b).

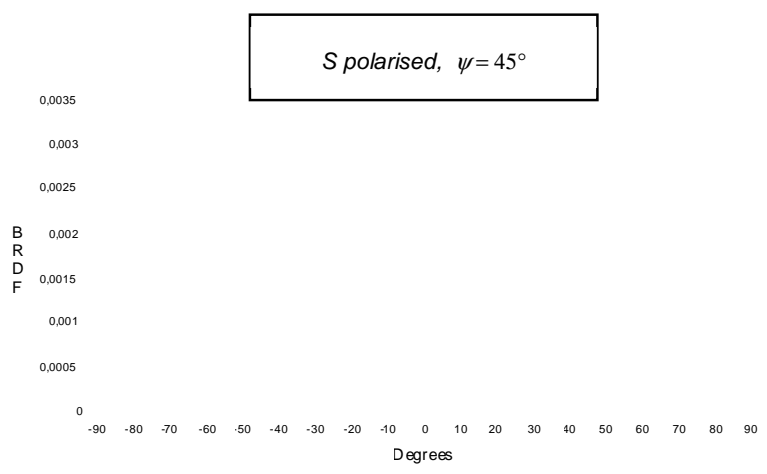

(a)

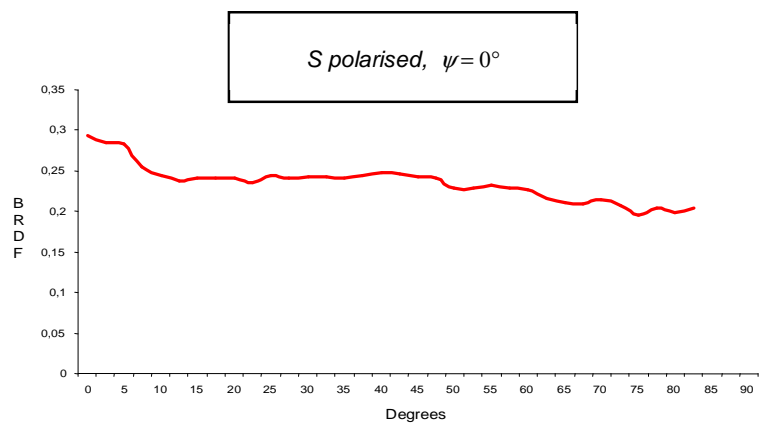

(b)
Fig. 44. BRDF of a predominantly diffuse reflection surface.

For comparison, Fig. 45 (a) and (b) show the BRDF of a highly specular reflector material.

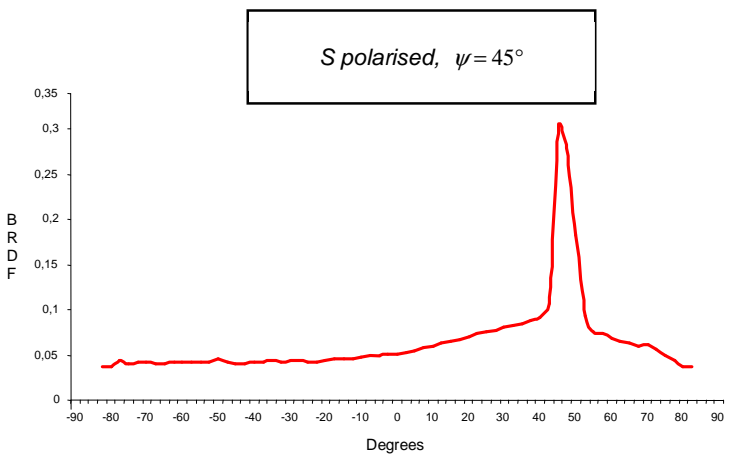

(a)

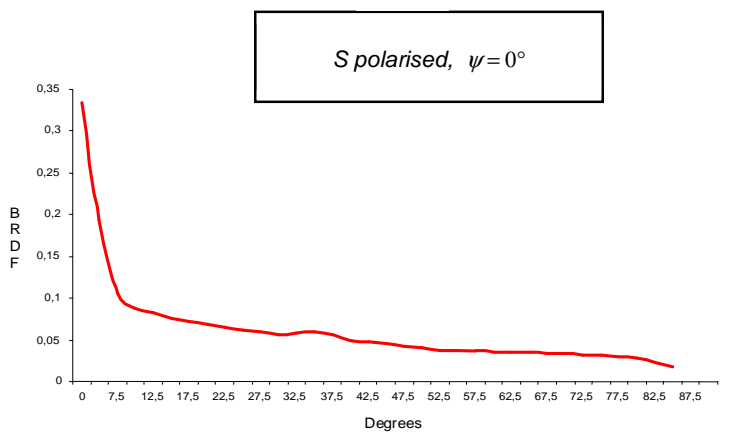

(b)

Fig. 45. BRDF of a predominantly specular reflection surface.

\subsubsection{Bandwidth}

The bandwidth of an electro-optic sensor can be determined by exposing the sensor to a tuneable highly-coherent radiation and analysing the output of the sensor. Whenever a tuneable emitter of highly coherent radiation is not available for the investigated wavelengths, a non-coherent emitter and a tuneable monochromator can be effectively used instead. It is important to note that the quality of the bandwidth measurement in both setups depend on the quality of prior calibration and precise setting of the wavelength.

\subsubsection{Detection threshold}

The STANAG 3733 titled: "Laser Pulse Repetition Frequencies (PRF) Used for Target Designation and Weapon Guidance", defines the LGW activation codes characteristics and the related tolerances. In order to determine the detection threshold of an electro-optic assembly, usually defined in terms of Minimum Detectable Power Density (MDPD), the following approach can be adopted. The laser output energy is progressively reduced using filters of increasing neutral optical densities. Adding various suitable filters, the MDPD is identified (i.e., the last nonzero reading in the sensor scale). The threshold value is recorded. The specific experimental setup for the determination of the MDPD of a laser seeker consists of two stages:

- Seeker activation codes generation (i.e., pulse duration, PRF and train); 
This is the author pre-publication version. This paper does not include the changes arising from the revision, formatting and publishing process. The final paper that should be used for referencing is:

R. Sabatini, M. A. Richardson, A. Gardi, S. Ramasamy, "Airborne laser sensors and integrated systems", Progress in Aerospace Sciences (2015), http: //dx.doi.org/10.1016/j.paerosci.2015.07.002i.

- Measurement of the MDPD (pulse) based on train energy measurements.

The experimental setup to determine the MDPD of a laser seeker is shown in Fig. 46.

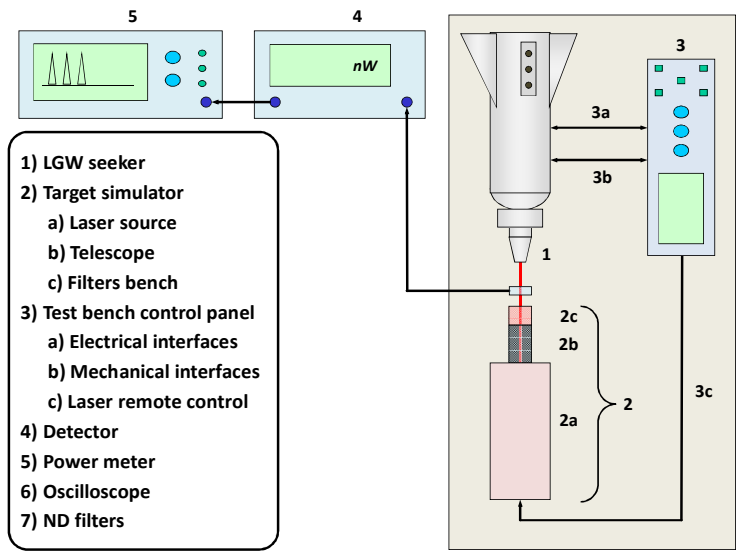

Fig. 46. Experimental layout for the measurement of the detection threshold of an LGW seeker.

\subsubsection{Test of protection filters}

During this research, a number of laboratory measurements are performed on various protection filters, in order to select the best available systems (i.e., optical density, transmittance in the visible, etc.). The assessment activities involve:

- ground personnel protection goggles;

- aircrew protection visors and spectacles;

- LTR Cinetheodolites Operator Sight (COS) filters.

In the last case, the optical gain of the $\operatorname{COS}$ needed to be determined first. For all systems, the principal objectives of the laboratory activities are:

- determination of the filters Optical Density (OD) at the specific operative wavelengths;

- determination of the transmittance in the visible.

\subsection{Ground experimental activities}

Ground experiments performed include Near Infrared (NIR) laser beam atmospheric propagation measurements, LTD/LRF pointing accuracy tests, systems harmonisation and performance evaluation trials of the laser test and evaluation range sensor tracking and measurement unit components. Furthermore, dedicated ground trials are performed for testing the obstacle avoidance system functions in order to assess its detection performance (in various weather conditions), and to verify the reliability of the obstacle classification algorithms.

Before performing ground and flight test activities using the laser obstacle and avoidance system, the laser sub-system is tested in the laboratory, in order to determine, against the manufacturer specification documents, the following characteristics:

- Average power transmitted;

- Pulse duration;

- Pulse Repetition Frequency (PRF);
- Laser beam misalignment with respect to the beamexpander support;

- $\quad$ Power consumption, Weight and Dimensions.

Ground trials of the system are performed in order to verify the system detection performance in various weather conditions, and to test the validity of the mathematical models used for performance calculations. This is particularly important for preparing the laser obstacle and avoidance system flight test activity. It is in fact necessary to define the criteria for determining the system detection range performances in the worst environmental conditions, and with the worst obstacle scenarios (i.e., small wires with low reflectivity), even without performing real tests in these conditions (i.e., using experimental data collected in fear weather and with average obstacles). Mathematical modelling and ground testing of the laser obstacle and avoidance system detection performance were therefore required in order to give proper weights to the parameters playing a role in realistic operational scenarios, and to determine the target laser obstacle and avoidance system detection performances to be demonstrated in flight. Fig. 47 illustrates this process.

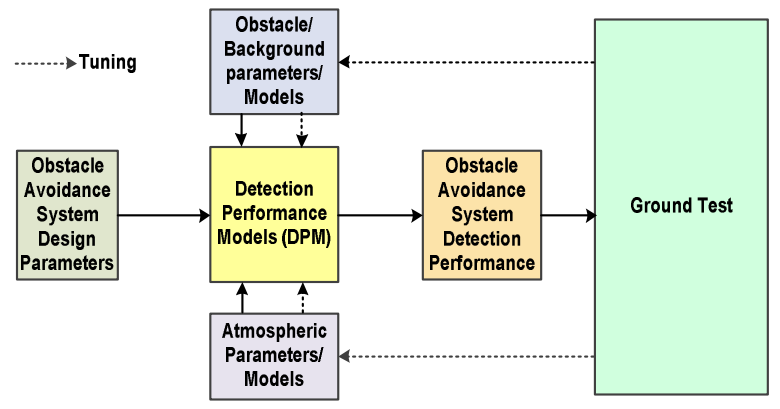

Fig. 47. Detection performance models and ground test.

As the ground test activities permitted to validate the models developed, it was then possible to identify reference sets of obstacle, background and atmospheric parameters giving the absolute minimum performance of the laser obstacle and avoidance system. This is illustrated in Fig. 48. Obviously, the successive flight test activities were performed only in a small portion of the laser obstacle and avoidance system operational envelopes, but the results obtained could be extended to the entire envelopes by using the validated mathematical models.

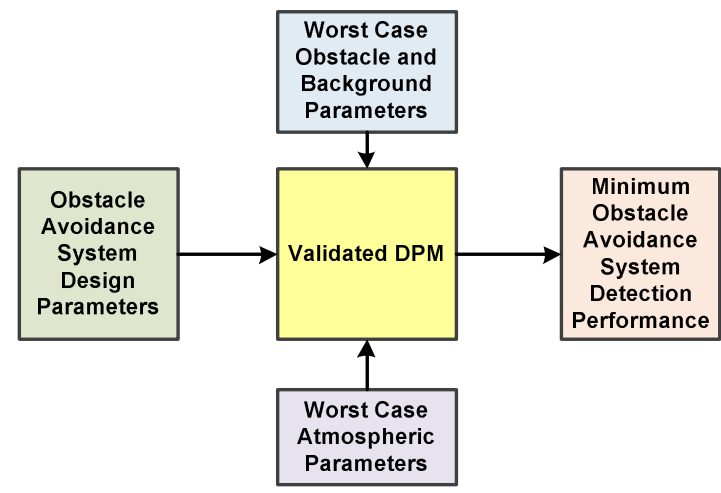

Fig. 48. Minimum detection performance calculation. 
This is the author pre-publication version. This paper does not include the changes arising from the revision, formatting and publishing process. The final paper that should be used for referencing is:

R. Sabatini, M. A. Richardson, A. Gardi, S. Ramasamy, "Airborne laser sensors and integrated systems", Progress in Aerospace Sciences (2015), http: //dx.doi.org/10.1016/j.paerosci.2015.07.002i.

\subsubsection{Frame rate optimisation}

If the sensor acquisition windows are not synchronised with the laser pulses incident on the target surface, the measure of the incident energy is completely unreliable. Good synchronisation is extremely difficult even at low PRF and almost impossible as the PRF increases. Therefore a preliminary study is required in order to determine optimal frame rates for the camera acquisition as a function of the known laser pulse parameters. The parameters describing the train of pulses are the pulse duration $(\tau)$, the pulse period $\left(T_{P}\right)$ and the PRF $(f)$. Similarly, the camera image acquisition process is defined by the frame period $\left(T_{F}\right)$ and the camera acquisition time $\left(T_{A}\right)$. Generally $T_{A}$ is inferior to $T_{F}$. The difference between $T_{F}$ and $T_{A}$ is the so called camera 'dark-time' $\left(T_{\text {dark }}\right)$. A schematic representation of the camera acquisition windows and dark zones is presented in Fig. 49.

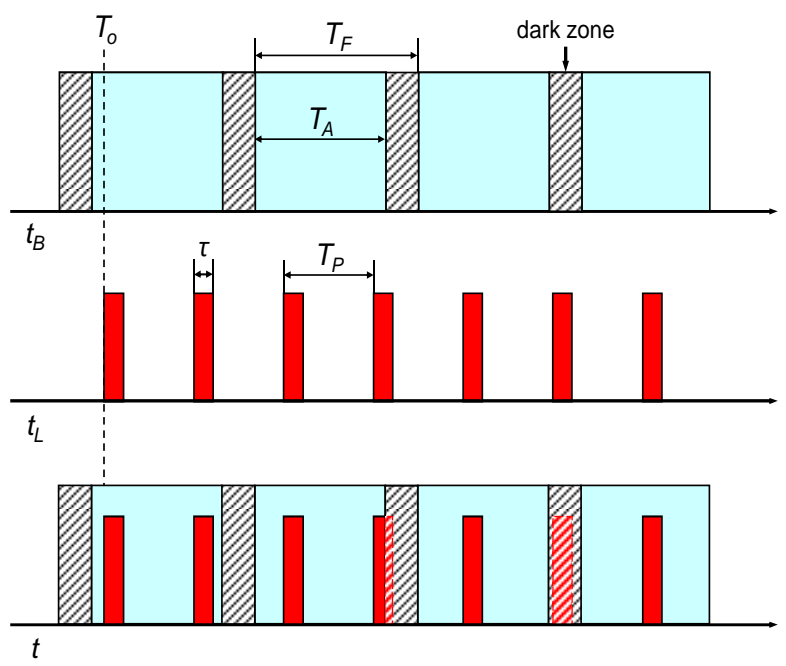

Fig. 49. NIR camera acquisition windows sequence and laser pulses.

Since the camera frames are not synchronised with the laser pulses, considering the camera acquisition windows sequence as time base $\left(t_{B}\right)$, the instant of arrival of the first laser pulse (reflected from the target) at the NIR camera $\left(T_{o}\right)$ can be treated as a random variable. Example results of a frame rate optimisation analysis, referred to laser emitters operating at $f=10$ $\mathrm{Hz}$ and $f=40 \mathrm{kHz}$ are summarised in Fig. 50 .

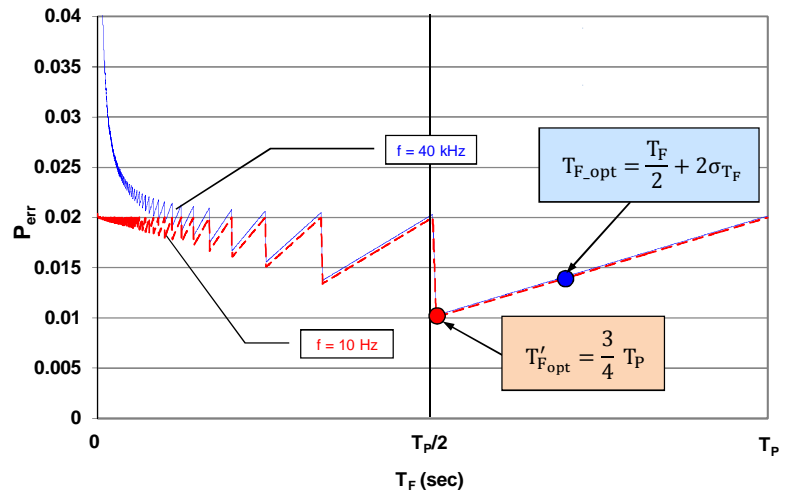

Fig. 50. Results of NIR camera frame rate optimisation analysis.

\subsubsection{Spot quality}

Linear and non-linear propagation phenomena are increasingly significant with longer propagation paths, leading to a noticeable spot spread. In order to investigate the effects induced by these phenomena, laser spot shapes and dimensions can be estimated by theory assuming a Gaussian energy distribution and considering 95\% of the total energy can be compared with the effective spot characteristics measured on the target. Using large data samples (i.e., 150 to 200 laser spots for each session), collected with various slant ranges and in various weather conditions, it is possible to obtain useful data about laser spot spreading and distortion characteristics at $\lambda=1064 \mathrm{~nm}$. An example of laser spot profile analysis is illustrated in Fig. 51.

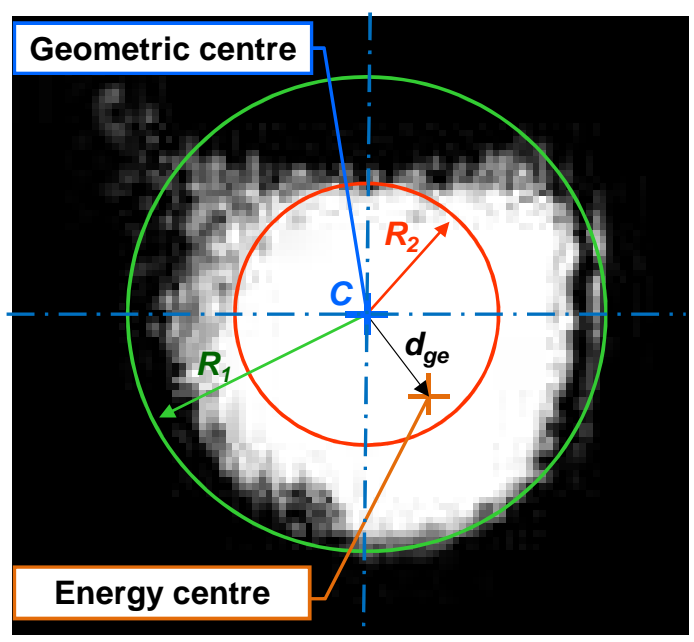

Fig. 51. Laser spot profile analysis [12].

The metrics used for spreading/distortion measurements with moderately and highly distorted laser spots are described hereafter. Using the 1/e divergence $\left(\Phi_{1 / e}\right)$ of the laser beam, the laser spot divergence at $95 \%$ of total energy can be computed as:

$$
\phi_{95 \%}=\frac{\phi_{1 / e}}{\sqrt{\frac{\ln (1-0.632)}{\ln (1-0.95}}}
$$

The 95\%-energy laser spot radius $(R)$ at a given distance $(d)$ can be estimated by:

$$
R=d \cdot \tan \phi_{95 \%}+a
$$

where $a$ is the output beam diameter. In order to define the laser spot distortion characteristics, the following spot measurable elements can be considered:

- Radius of the smallest circle inscribing the entire spot $\left(\mathrm{R}_{1}\right)$;

- Radius of the smallest circle, centred in the spot geometric centre $\mathrm{C}$, contained by the spot image $\left(\mathrm{R}_{2}\right)$;

- Distance between energetic and geometric centres $\left(\mathrm{d}_{\mathrm{ge}}\right)$. These spot characteristics are used to conveniently describe the spot quality in terms of spreading and distortion. Based on them, the following Spot Distortion Parameters (SDP) are defined:

$$
\begin{gathered}
Q_{S}=\frac{R}{R_{1}} \\
Q_{D e}=1-\frac{d_{g e}}{R_{1}}
\end{gathered}
$$


This is the author pre-publication version. This paper does not include the changes arising from the revision, formatting and publishing process. The final paper that should be used for referencing is:

R. Sabatini, M. A. Richardson, A. Gardi, S. Ramasamy, "Airborne laser sensors and integrated systems", Progress in Aerospace Sciences (2015), http: //dx.doi.org/10.1016/j.paerosci.2015.07.002i.

$$
Q_{D g}=\frac{R_{2}}{R_{1}}
$$

The parameter $Q_{S}$ describes the spot spreading, $Q_{D e}$ is relative to the energy profile distortion and $Q_{D g}$ is relative to the geometric distortion of the laser spot. Conveniently, the SDP parameters were so that they equated to 1 in the ideal Gaussian case and tend to 0 in the worst case. For laser spots preserving a shape almost circular and an energy profile approximately Gaussian (like the original laser signal at the system aperture), the geometric centre is computed as the centre of the smallest circle inscribing the laser spot. The spot energy centroid (maximum of the laser energy) is determined by using a dedicated interpolation function. In all cases, the position of the geometric and energetic centres is referenced to the target bi-dimensional Cartesian frame (i.e., horizontal/vertical scales and origin at the target centre). By firing the laser beam exactly at the centre reticle of the target, the geometric and energetic pointing errors can be determined as the RSS of the horizontal and vertical error components. During these measurements, the relevant atmospheric parameters can be recorded (i.e., visibility, temperature, relative humidity, wind intensity/direction, etc.) allowing correlation with the linear and non-linear propagation models.

\subsubsection{Boresight and bearing/pointing accuracies}

In order to assure accurate azimuth and elevation readings, electro-optic assemblies are tested in boresight and bearing/pointing accuracies. The procedure involves the installation of the assembly on a high-precision 2-degrees-offreedom goniometer stage and the adoption of a target at distance. The assembly is rotated using the goniometer stage to various predetermined azimuth and elevation orientations, and the readings to the target centre reticle are recorded. The boresight is assessed by setting the null relative elevation and azimuth from all directions and recording the reading to the target centre reticle. The pointing accuracy data (i.e., displacement of the energetic and geometric centres of the laser spots on the target with respect to the target centre) were obtained using the procedures described below for the three cases of slightly distorted, highly distorted and broken laser spots.

\subsubsection{Field of view}

Analogously to the boresight and bearing/pointing accuracy measurements, the field-of-view of an electro-optic assembly can be determined by installing the assembly on a 2-degrees-offreedom goniometer stage and adopting a target at distance and a collimator. The assembly is slewed alternately in azimuth and elevation using the goniometer stage and the extreme angles at which the target is visible are recorded.

\subsubsection{Angular resolution}

The angular resolution of an electro-optic sensor can be accurately determined by adopting resolution pattern arrays as targets, such as the one depicted in Fig. 52, and by accurately measuring the distance between the target and the sensor. A common choice for the precise measurement of the distance to the target is the LRF. By recording the finest resolution pattern discernible at varying distances between the target and the sensor, it is ultimately possible to correlate range and resolution and hence identify the angular resolution with high precision.

Resolution testing of airborne passive IR systems can be performed by employing target panels such as the one shown in Fig. 53. Adequate coatings and calibrated thermocouples ensure that the vertical/horizontal stripes present a high contrast both in the visible and infrared portions of the spectrum.

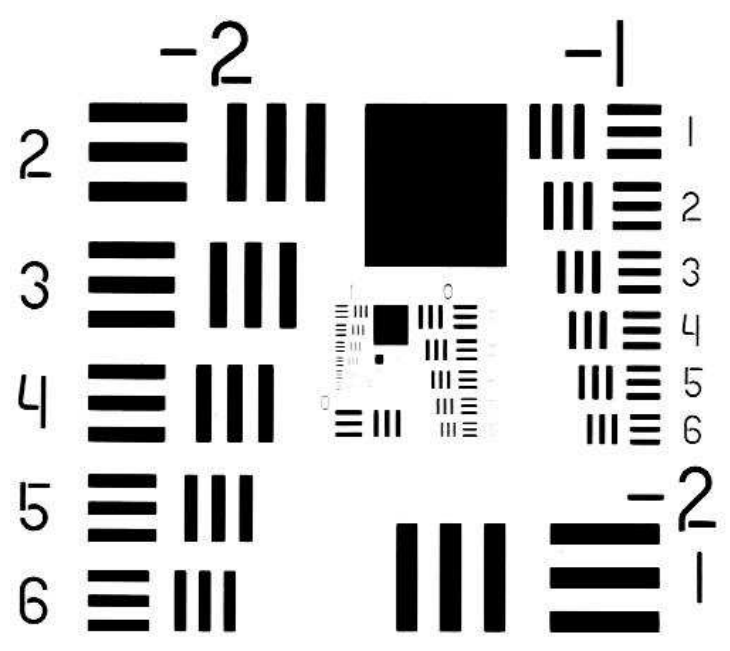

Fig. 52. Detail of the resolution pattern adopted by the US Air Force in 1951.

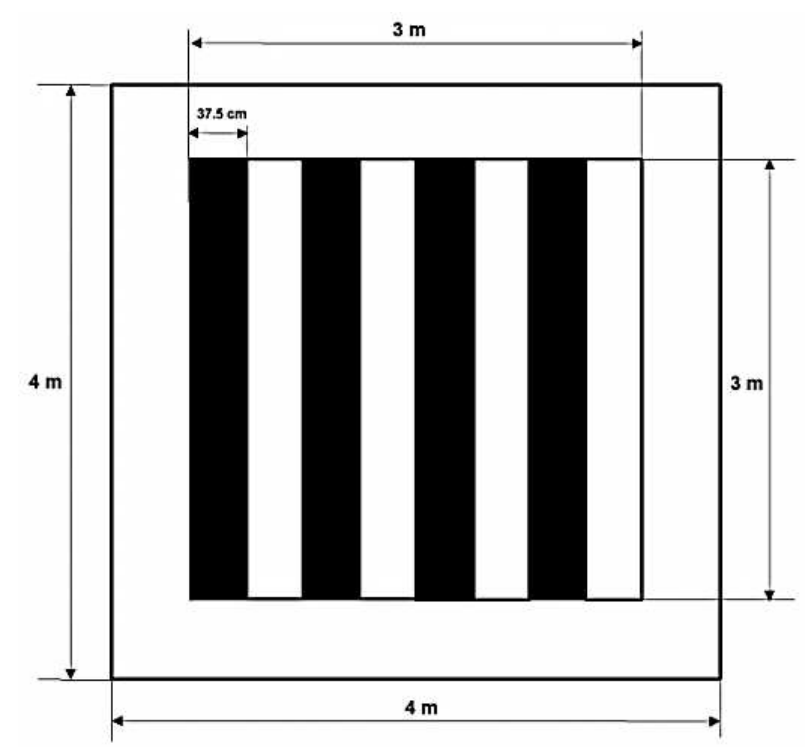

Fig. 53. High thermal contrast resolution panel [163].

\subsection{Flight test activities}

Most laser system test activities are carried out during laboratory and ground sessions. However, a number of flight sorties are performed in order to verify the operational compliance of the systems with aircraft and missions requirements. This activity also aids the personnel involved (aircrews and engineering officers) in gaining confidence with the laser systems during their operational employment. Particularly, the laser emitter, sensors and target systems as well as the mission monitoring and control station are tested during their employment in real air-to-ground missions (both with and without deliveries of guided weapons). Flight test activities that are therefore performed as part of the laser system test and evaluation activities typically address the following: 
This is the author pre-publication version. This paper does not include the changes arising from the revision, formatting and publishing process. The final paper that should be used for referencing is:

R. Sabatini, M. A. Richardson, A. Gardi, S. Ramasamy, "Airborne laser sensors and integrated systems", Progress in Aerospace Sciences (2015), http: //dx.doi.org/10.1016/j.paerosci.2015.07.002i.

- range performances;

- adverse weather operability;

- pointing accuracy;

- $\quad$ spot quality;

- $\quad$ spot energy profile;

- codes characteristics;

- atmospheric extinction measurements;

- propagation measurements in oblique air-to-ground paths;

- where a Forward Looking System (FLS) is present, combined FLS/laser system performance evaluation.

Flight test is an exceptionally important data gathering vehicle to validate the performance of airborne laser systems. However, the advantages offered by flight trials are paid in terms of a dramatic increase in development costs. Therefore, a tailored philosophy has to be adopted in order to reduce the costs associated with the development process and to obtain the higher possible level of efficiency. In most cases, instead of carrying out flight trials at the end of the systems integration process, it is important to involve flight test personnel (flight test engineers, test pilots, etc.) in the various system design and integration phases, also allowing participation of design and integration engineers to the flight test planning activities. Specialised personnel involved typically includes:

- $\quad$ Flight Test Engineers (FTEs);

- System/Hardware Engineers (SYEs);

- Software Engineers (SWEs);

- Experimental Test Pilots (ETPs).

Their participation to the definition of sub-system basic functional requirements and specific test requirements, software/hardware development and testing, and an extensive use of simulation tools at the various levels allows a continuous interaction throughout the development processes and a consequent definition of a 'balanced' test matrix. As a result, test items allocated to flight trials sensibly reduce according to a sharper and a more careful selection of not redundant and significant test items, aimed at calibrating simulation models to allow a more accurate prediction of physical phenomenon and possible operational deviations. In this perspective, flight trials are not considered the final step of the system operability/reliability verification process, but became an important portion of the development process itself (Fig. 54).

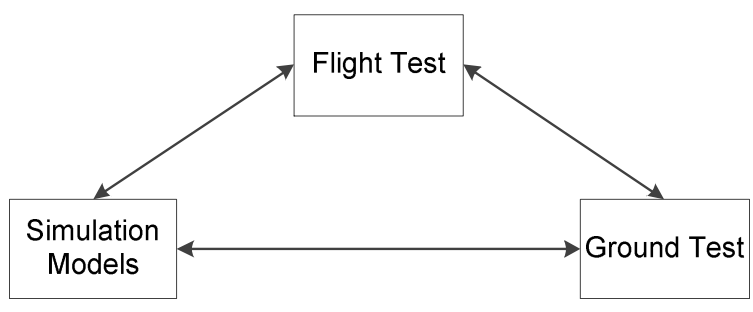

Figure 54. Laser Systems Test Philosophy.

Based on the literature, this test methodology demonstrates improvements in efficiency and optimization of data gathering. A consequent reduction of costs and time is therefore experienced in the process.

\subsubsection{Test Requirements and Methods}

Once the electrical/mechanical compatibility of the laser subsystems with the aircraft is assured, the following types of test must be carried out:

- Software developmental testing (Phase D);

- Flight mechanics testing (handling, vibrations, etc.);

- Hardware integration and sub-system testing (electromagnetic compatibility, power management, etc.).

Additionally, in the case of LGWs, store (safe) separation testing must be accomplished. Test requirements, mathematical models and analysis tools provide guidelines for defining the aircraft in flight data acquisition, telemetry systems and the related list of parameters to be recorded during tests. Although some sensors are typically fitted to the aircraft structure to detect vibrations and other aeromechanical parameters, the avionics system is the main source of flight data (computer digital streams, avionics and weapon buses, serial lines tapping, videos and cameras). Simulation tools typically used during the experimental activities, and progressively improved as a flight test activity spin-off, included the following:

- Aerodynamic Simulation;

- Masking Analysis and Simulation;

- System Performance Simulation;

- Laser Safety Areas (if applicable).

Additionally, the following tests must be accomplished for LGW:

- Store Separation Simulation;

- Aircraft Weapon Aiming Simulation;

- Ballistic Safety Areas.

For external laser payloads, Computational Fluid Dynamics (CFD) and Computer Assisted Design (CAD) codes are used in the aerodynamic calculations process. An example of the process steps is presented in Fig. 55 and described below:

- Definition of a conceptual model (from the master geometry data base or other already assessed geometry);

- Build up in CAD/CATIA of a derived geometry model (by translating a series of points in polynomial entities) congruent to that defined in the previous step;

- Possible simplification of the geometry depending on the specific aircraft area to be analysed;

- Transfer of the geometrical data (polynomial coefficients) from CATIA to the input files of CFD codes with the appropriate format. 
This is the author pre-publication version. This paper does not include the changes arising from the revision, formatting and publishing process. The final paper that should be used for referencing is:

R. Sabatini, M. A. Richardson, A. Gardi, S. Ramasamy, "Airborne laser sensors and integrated systems", Progress in Aerospace Sciences (2015), http: //dx.doi.org/10.1016/j.paerosci.2015.07.002i.

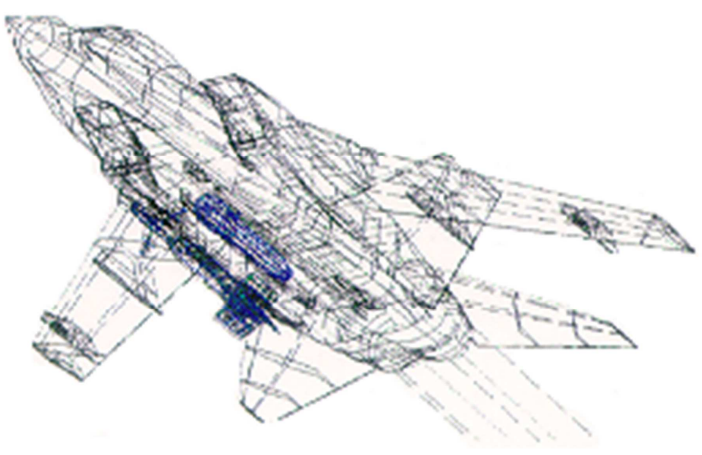

a. Original Geometry (CATIA Surfaces)

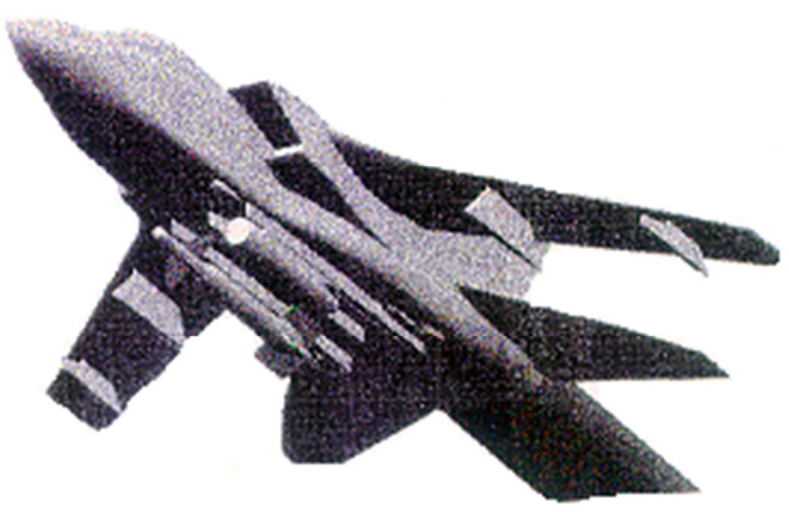

b. Solid Model (CATIA Contest)

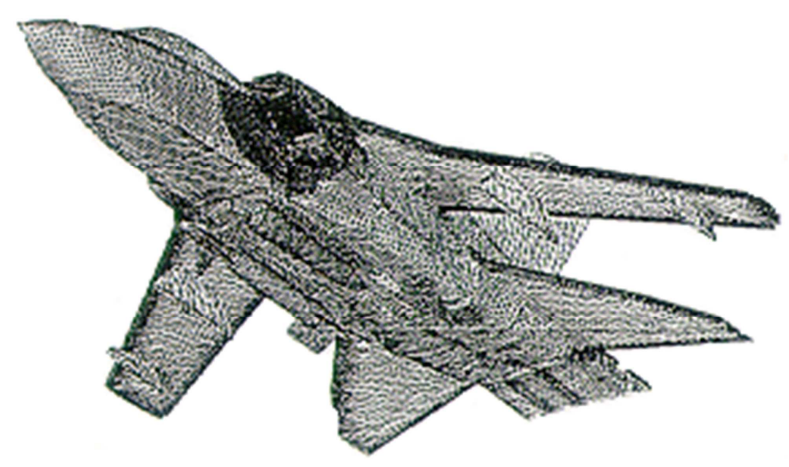

c. Surface Grid (SUR 3D Code)

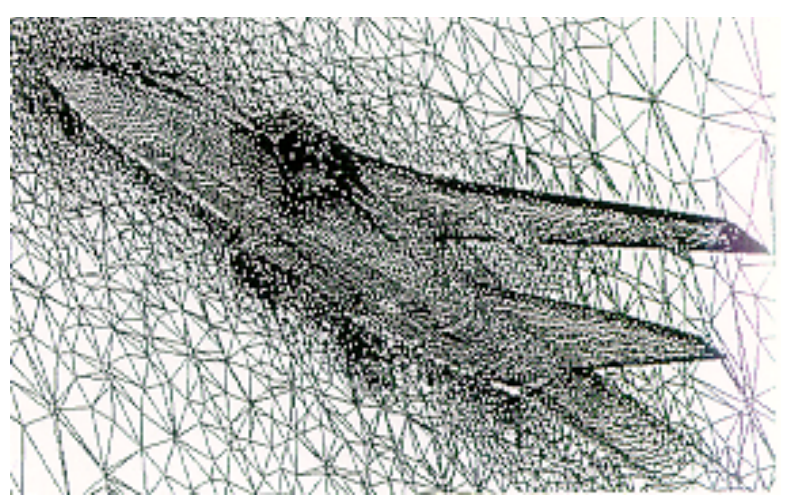

d. Spatial Grid (M3D Code)

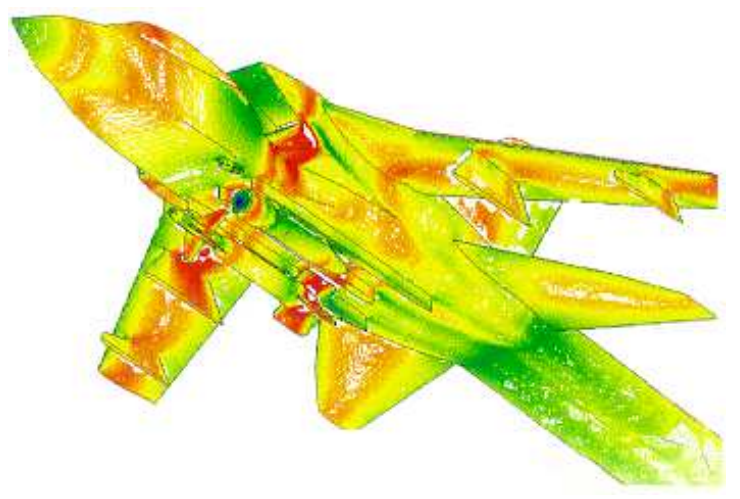

e. ISO-MACH Euler Results (UES3D Code)

Fig. 55. CATIA-CFD Interface in Aerodynamic Simulation.

Furthermore, a 3D Euler flow solver is used to find the flow field stationary solution of a three dimensional compressible inviscid fluid by using a pseudo-unstationary method in time and spatial finite volume method on unstructured tetrahedral meshes. During application of the 3D Euler code the following steps are performed:

- Generation of surface and spatial grids to produce the flow field discretization to be used by the analysis code;

- Numerical results from Euler Equation Solutions (UES3D code) and analysis of these results;

- Optimisation of the model on the basis of the result analysis and consequent verification with numerical code;

- Final assessment and loading of the new model in the master geometry database.

The above described methodology represents the standard procedure adopted in the whole aero-design process, for external stores integration on aircraft. Particularly, it permits to carry out aero-analysis with strongly representative models (i.e., mathmodels from the assessed geometry), and allows to quickly and correctly optimise the geometrical model utilised for the aeroanalysis. The optimised geometry can be easily re-inputted to the master geometry database. Additionally, the described methodology can be also applied to the aero-analysis required for trajectory computations. For integration of external stores in a combat aircraft, the analysis of store separation trajectories is aimed at defining the safe release envelope. Fig. 56 shows the complete flow diagram of the activities performed to achieve the Final Operational Clearance (FOC) for the store safe separation. As shown in the flow diagram, the store trajectory calculation is influenced by the following parameters:

- Aircraft flight conditions;

- Sore mass and inertia characteristics;

- Sore aerodynamic coefficients (free air);

- Aircraft store aerodynamic interference (with loads installed);

- Aircraft flow field;

- Ejector Release Unit (ERU) performance;

- Store physical constraints. 
This is the author pre-publication version. This paper does not include the changes arising from the revision, formatting and publishing process. The final paper that should be used for referencing is:

R. Sabatini, M. A. Richardson, A. Gardi, S. Ramasamy, "Airborne laser sensors and integrated systems", Progress in Aerospace Sciences (2015), http: //dx.doi.org/10.1016/j.paerosci.2015.07.002i.

Amongst the above parameters, the aerodynamic data are those having the major influence on store separation behaviour and demanding the greater effort for their determination.

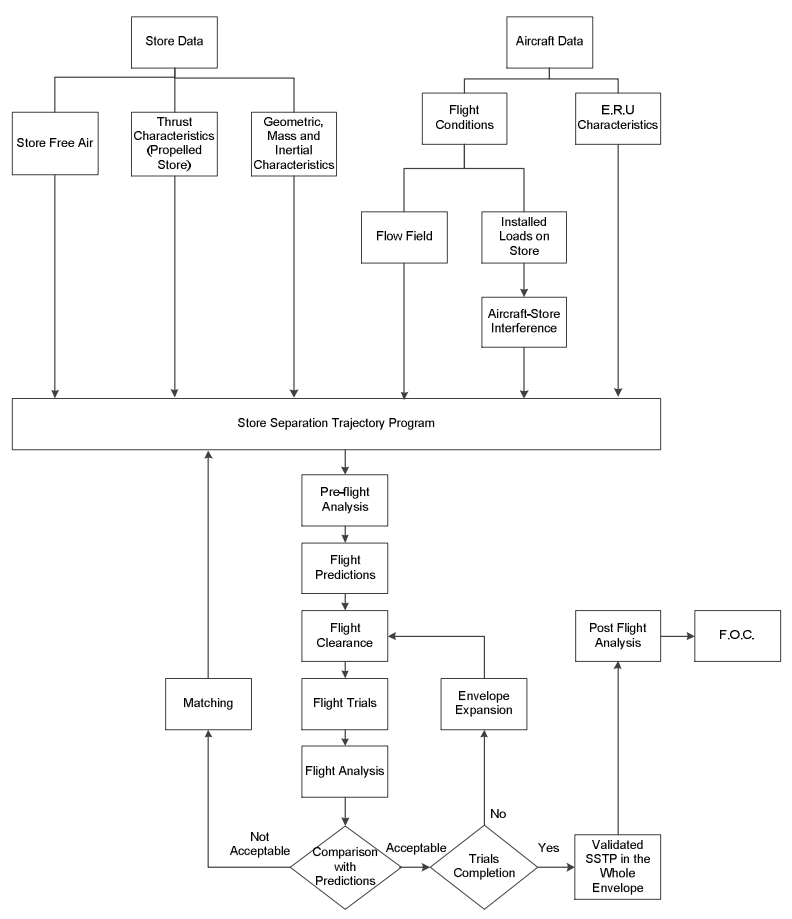

Fig. 56. Store Integration Activities (Safe Separation Aspects).

The models used for system performance calculation in the test phase are the same models described above for system design and integration:

- Laser Range Equation. A convenient form of the Laser Range Equation (LRE). This equation established a relationship between all key physical parameters (i.e., geometry, propagation, reflection, systems characteristics).

- Atmospheric Propagation Model. A laser propagation model covering the cases of practical interest just be used. This model allows calculation of the atmospheric extinction as a function of observable meteorological parameters (visibility, absolute humidity, rainfall-rate, etc.) in the transmitting/receiving paths.

- Geometric Model. A detailed mission geometry model covering all cases and system functionalities of practical interest. For LGWs, the geometric model included technical assumption about the LGWs guidance characteristics and considerations related with the geometry of self-designation and co-operative attacks.

- Reflection Model. For most performance calculation purposes, the target surface can be assumed as a perfect diffuser scattering incident light equally in all directions. For such ideal surfaces, the intensity $(\mathrm{W} / \mathrm{m} 2)$ of diffuse reflected light is given by the Lamber equation. A more realistic reflection model can be used (e.g., Phong diffusespecular reflection model) with the purpose of identifying optimal bomb-aircraft relative directions for performing successful LBG attacks.
In some cases, it is important to experimentally measure the laser beam atmospheric extinction in various weather conditions. These measurements typically include:

- Determination of atmospheric extinction with different visibilities, temperatures, relative humidity values, wind intensities/directions, etc.;

- Determination of atmospheric extinction with different types and amount of precipitation.

Various alternative techniques that were successfully applied for the in-flight measurement of atmospheric propagation are [12, 55]. Most recently, these include:

- Measurement technique employing STU instrumentation (i.e., non-calibrated NIR camera and PEP sensors): this technique is based on direct measurements of laser energy at pre-defined locations on the target (PEP sensors) and use of the non-calibrated NIR camera spot frames to reconstruct, by means of the pixel intensity matrixes (associated to each frame), the overall energy intensity profile, from which atmospheric extinction is ultimately computed.

- Measurement technique employing a calibrated NIR camera only: this technique involves a preliminary calibration (that may be performed in the laboratory using an integrating sphere or on ground in the test range) and successive adoption of a dedicated energy profiling function implemented within the camera post-processing software. The energy profiling function permits to obtain the total spot energy by directly converting pixel intensity data into energy measurements. Using these measurements, atmospheric extinction is computed.

- Measurement techniques specifically developed for merged emitter-receiver systems.

During the measurements, a number of atmospheric parameters are monitored and recorded including meteorological visibility $(V)$, temperature $(T)$, Relative Humidity $(R H)$, atmospheric pressure $(P)$, wind direction and velocity $\left(W_{d}\right.$ and $\left.W_{v}\right)$, solar radiation $\left(E_{s}\right)$, and cloud amount.

\subsubsection{Flight Test Instrumentation and Experimental Measurements}

As depicted in Fig. 57, a typical flight test facility for airborne laser systems is composed by the following main systems:

- Permanent and/or modular target(s);

- Permanent/movable Sensor Tracking and measurement Unit (STU);

- Monitoring and control Station Unit (MSU);

- Network connectivity (between MSU and STU);

- Voice/Data/Video-link connectivity (aircraft to MSU);

- Precision positioning/navigation systems, such as Differential GPS/GNSS (DGPS/DGNSS);

- Meteorological sensor units. 
This is the author pre-publication version. This paper does not include the changes arising from the revision, formatting and publishing process. The final paper that should be used for referencing is:

R. Sabatini, M. A. Richardson, A. Gardi, S. Ramasamy, "Airborne laser sensors and integrated systems", Progress in Aerospace Sciences (2015), http: //dx.doi.org/10.1016/j.paerosci.2015.07.002i.

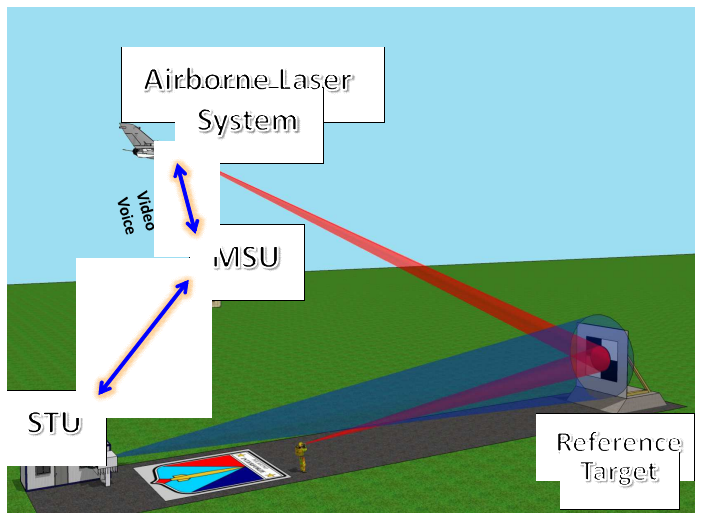

Fig. 57. Concept of Operations. Adapted from [55].

Airborne laser systems are aimed towards the target by the aircrew operators. A Laser Safety Officer (LSO) is responsible of verifying that the airborne laser system is safely aimed within the target area and that the target lock-on status has been achieved by the airborne system. The LSO is in charge of authorising the activation of the airborne laser system. The authorisation might be given also upon receiving confirmation (via voice/data link) from the airborne operators that the target has been unambiguously recognised and aimed to, if regulations contemplate such option. A number of ground-based sensors, typically arranged within a movable Sensor Tracking and measurement Unit (STU), perform various measurements associated with the incident laser energy along the entire flight test time span, such as the laser spot centroids, their relative displacement from the target centre (global pointing error), the spot shape and the spatial energy distribution. All applicable measurements are recorded. During the laser activation, the STU sends the relevant laser spot parameters (i.e., dimension and position with respect to the target centre) to a Monitoring and control Station Unit (MSU). Relevant mission and measurement parameters are shown on displays within the MSU to allow the LSO to supervise the operations. When the laser spot approaches the target peripheral zones and the LSO believes there is a possibility for the laser beam to fall outside the target, he orders the airborne operators to deactivate the laser (through the voice/data link). The targets consist of the most adequate surface coatings depending on the test activity and the type of laser employed. For instance, IR targets should consist of multiple bars, heated at precisely tuneable temperature differences $(\Delta T)$ with respect to the background. The target dimensions and shapes are defined depending on the mission parameters and, in case of military use, on the real targets they are meant to emulate. The STU should be positioned nearby the targets, possibly in a protected location. The STU is composed by the following elements:

- Sensor/Detector arrays and processing hardware;

- Signal acquisition and processing software;

- Computing and recording systems;

- IR and TV cameras.

The STU continuously measures the position of the laser spot energy centroid with respect to the target centre. A representation of this position within the target is supplied in real-time to the LSO. The STU also determines and records the geometric dimensions of the laser spot on the target. Based on the employed sensors, the STU performs a number of laser energy measurements (on the target) and therefore allows the MSU to calculate several derived metrics as part of the post-processing. For instance, the impact of atmospheric and operational factors on the laser beam can be derived when required. Active and/or orientating sensors receive dedicated energy supply and are controlled by dedicated control electronics. Outputs are fed through the network. The MSU subsystem includes the network interfaces, the processing units, the laser data presentation and recording, as well as other devices for real-time monitoring and post-mission analysis of the experimental data. The MSU receives data from the STU and shows, on dedicated displays, the laser spot on the target and the video signal received from the aircraft (video-link). Typically, the MSU should be capable of:

- simultaneously showing output data from multiple sensors;

- providing aural/visual warnings to the LSO when the expected laser signal is not detected by the STU;

- showing in real-time the video signal received from the aircraft (if available), on dedicated displays.

The MSU may also accommodate suitable safety devices for the remote deactivation of the airborne laser system. The MSU is typically composed of:

- high performance post-processing hardware with analogue and digital I/O and networking interfaces;

- state-of-the-art post-processing software;

- recording facilities;

- Voice/Data/Video-link communication facilities.

When it is necessary to determine the effects of atmospheric transmittance, the flight test facility should be equipped with meteorological units, including the sensors necessary for accurate measurement of temperature $(T)$, pressure $(P)$, wind speed $\left(W_{s}\right)$, relative humidity $(R H)$, rainfall rate $(\Delta x / \Delta t)$, and turbulence structure constant $\left(C_{n}\right)$. An example of a large-size target is shown in Fig. 58.
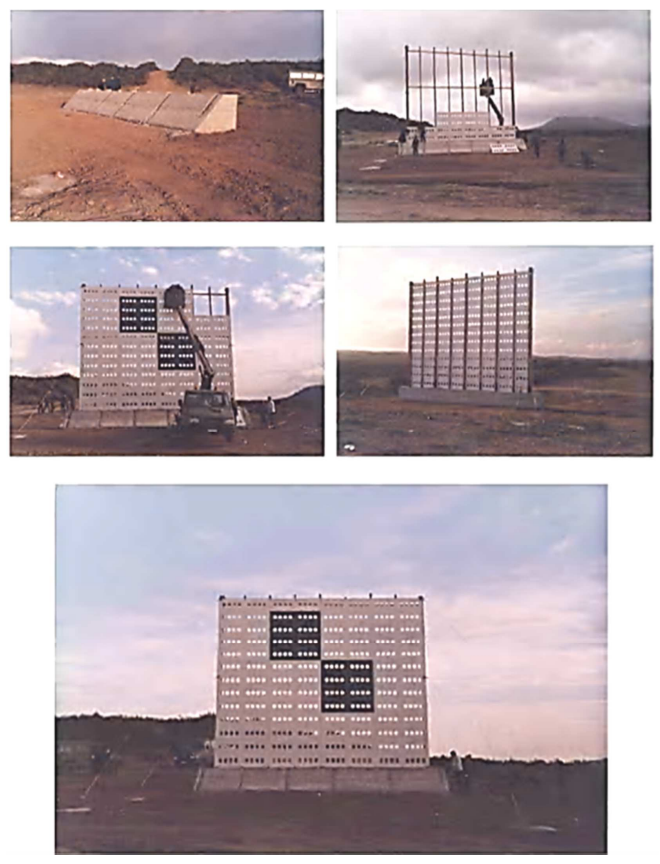

Fig. 58. Fixed fast recoverable target [12]. 
This is the author pre-publication version. This paper does not include the changes arising from the revision, formatting and publishing process. The final paper that should be used for referencing is:

R. Sabatini, M. A. Richardson, A. Gardi, S. Ramasamy, "Airborne laser sensors and integrated systems", Progress in Aerospace Sciences (2015), http: //dx.doi.org/10.1016/j.paerosci.2015.07.002i.

A detachable target panel module with holes for photodetectors is shown in Fig. 59.

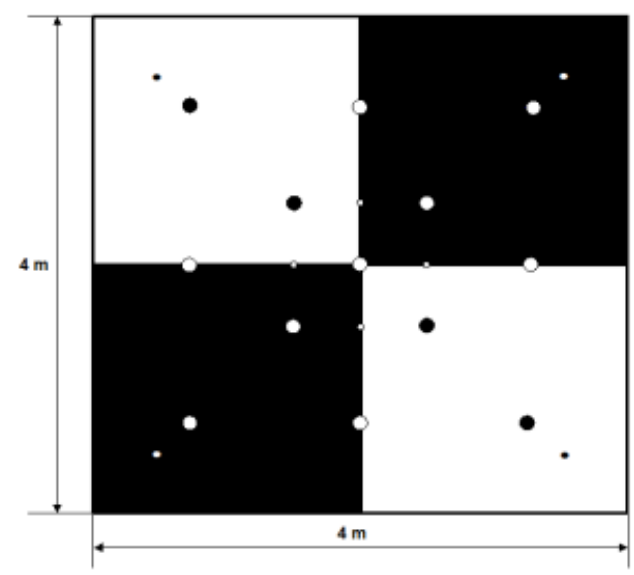

Fig. 59. Detachable target panel module [163].

When the test campaign requires the determination of the incident laser energy, a number of alternative energy measurement techniques can be adopted to determine the total incident laser energy and, subsequently, the atmospheric transmittance [12]. For instance, a video-camera pre-calibrated for radiance can be used in conjunction with a highly selective filter (laser wavelength) to detect the laser spot energy on the target and to generate a Pixel Intensity matrix (PIM) in high resolution greyscale format. The IR-camera calibration is an experimental procedure that allows determination of the Integrated Radiance Response Function (AIRF). As described in [163], the laboratory calibration procedure can be accomplished by using an integrating sphere. The layout of such calibration procedure is reproduced in Fig. 60.

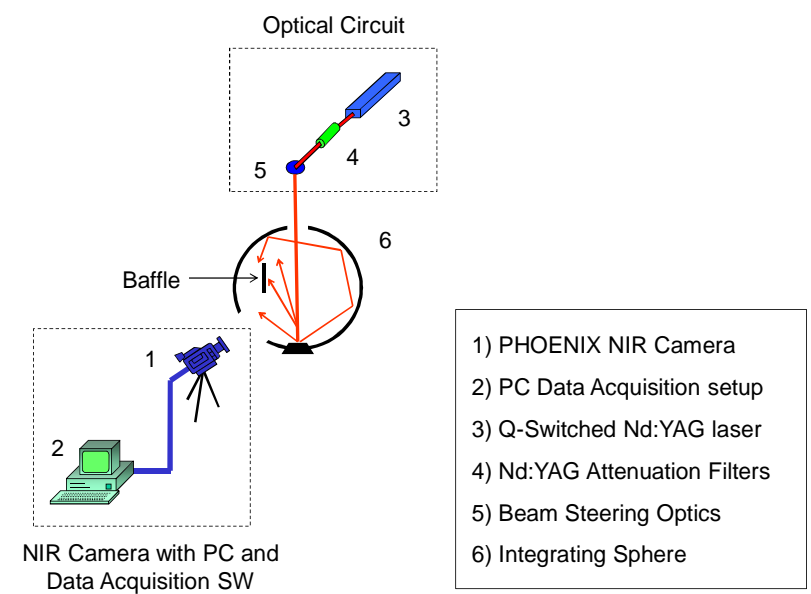

Fig. 60. Layout of a NIR camera laboratory calibration setup by means of integrating sphere. Adapted from [12].

The response of a single pixel in terms of Analogue Digital Unit (ADU) is:

$$
\mathrm{ADU}_{\mathrm{i}, \mathrm{j}} \propto \frac{\mathrm{A}}{4 \cdot \mathrm{f} \#^{2}+1} \cdot \mathrm{g} \cdot \mathrm{i}_{\text {time }} \cdot \int_{\lambda 1}^{\lambda 2} \tau_{\lambda} \eta \cdot \mathrm{E}_{\lambda} \mathrm{d} \lambda
$$

where:

$$
\begin{array}{ll}
\lambda & =\text { wavelength } \\
\lambda_{1}, \lambda_{2} & =\text { limits of the IR-camera spectral band filter } \\
\eta_{\lambda} & =\text { detector quantum efficiency } \\
E_{\lambda} & =\text { spectral radiance } \\
\tau_{\lambda} & =\text { optics transmittance } \\
A & =\text { pixel area } \\
g & =\text { read-out electronics gain } \\
f \# & =\text { optics f-number } \\
i_{\text {time }} & =\text { IR-camera integration time }
\end{array}
$$

where $\lambda$ is the wavelength, $\lambda_{1}$ and $\lambda_{2}$ are limits of the IR-camera spectral band filter, $\eta_{\lambda}$ is the detector quantum efficiency, $E_{\lambda}$ is the spectral radiance, $\tau_{\lambda}$ is the optics transmittance, $A$ is the pixel area, $g$ is the read-out electronics gain, $f \#$ is the optics $\mathrm{f}$-number, $i_{\text {time }}$ is the video-camera integration time. Therefore, the experimental parameters to be controlled during the calibration procedure are the integration time, the optics $f$-number and other settings of the video-camera (e.g., the gain of the readout electronics which may be selected by the operator). By fixing these parameters for a certain interval of integral radiance, it is possible to determine the AIRF of the camera by using an extended reference source. The function (calibration curve) so obtained is then used to determine the values of integral radiance for reconstructing the radiant intensity map of the target. The energy measurement technique employing a video-camera precalibrated for radiance is shown in Fig. 61.

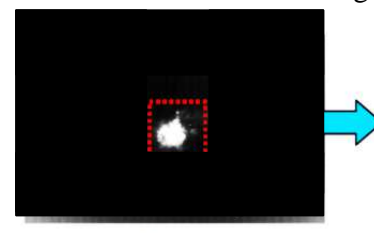

Target Reference Frame
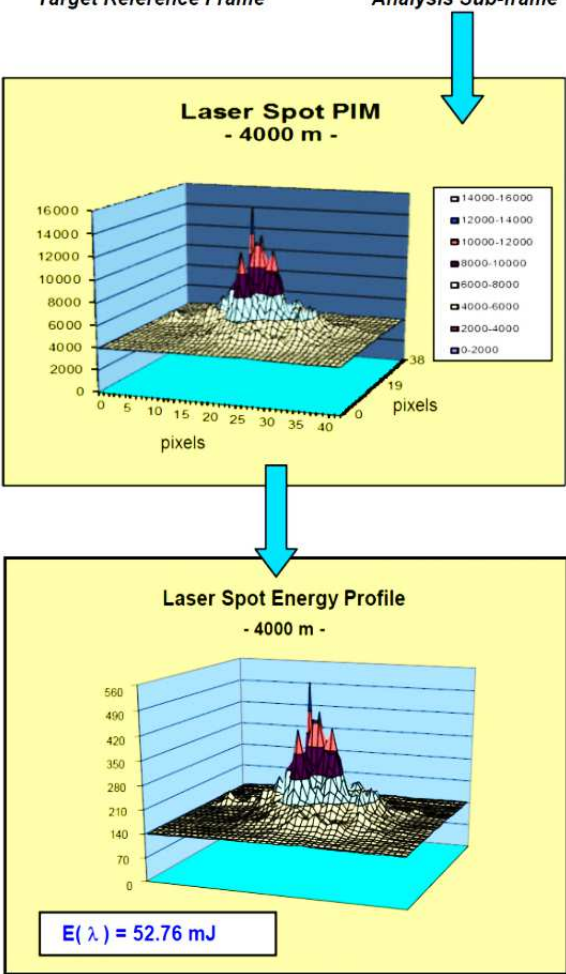
This is the author pre-publication version. This paper does not include the changes arising from the revision, formatting and publishing process. The final paper that should be used for referencing is:

R. Sabatini, M. A. Richardson, A. Gardi, S. Ramasamy, "Airborne laser sensors and integrated systems", Progress in Aerospace Sciences (2015), http: //dx.doi.org/10.1016/j.paerosci.2015.07.002i.

Fig. 61. Energy measurement technique employing a video-camera precalibrated for radiance.

No calibration is required if an array of detectors performs direct measurements of the incident laser spot energy at specific locations on the target surface (Fig. 62).
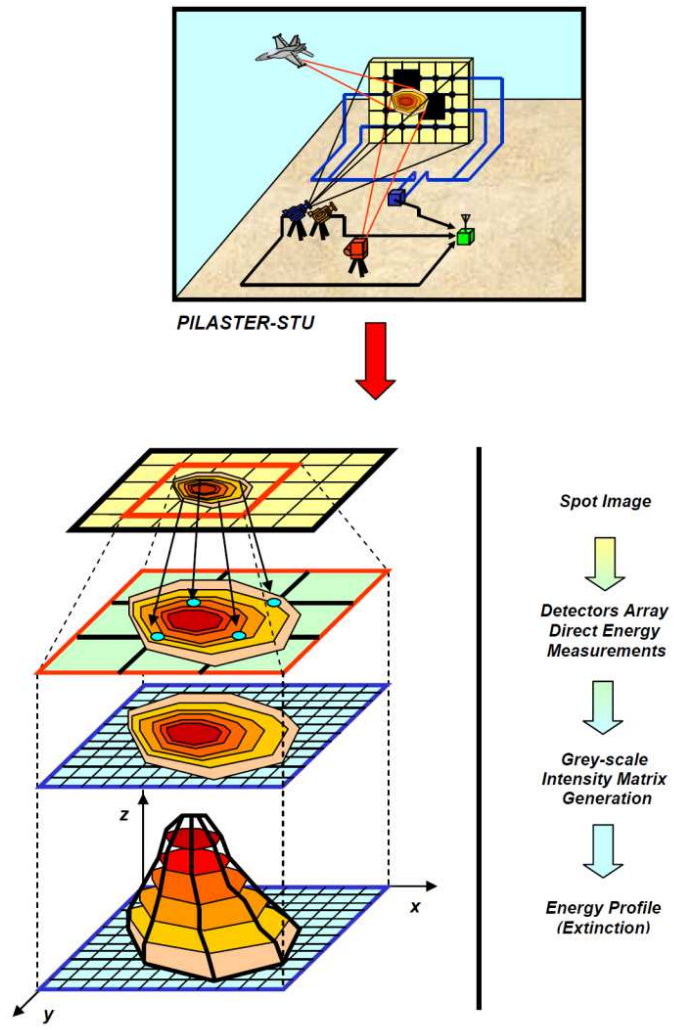

Energy Profile (Extinction)
Fig. 62. Energy measurement technique employing a non-calibrated video-camera and an array of photodetectors.

An additional technique can be adopted for high Pulse Repetition Frequency (PRF) applications (beyond $10 \mathrm{kHz}$ ), where the videocamera frame-grabbing performance is exceeded and other energy measurement techniques may not be employed. One of the possible experimental arrangements is depicted in Fig. 63.

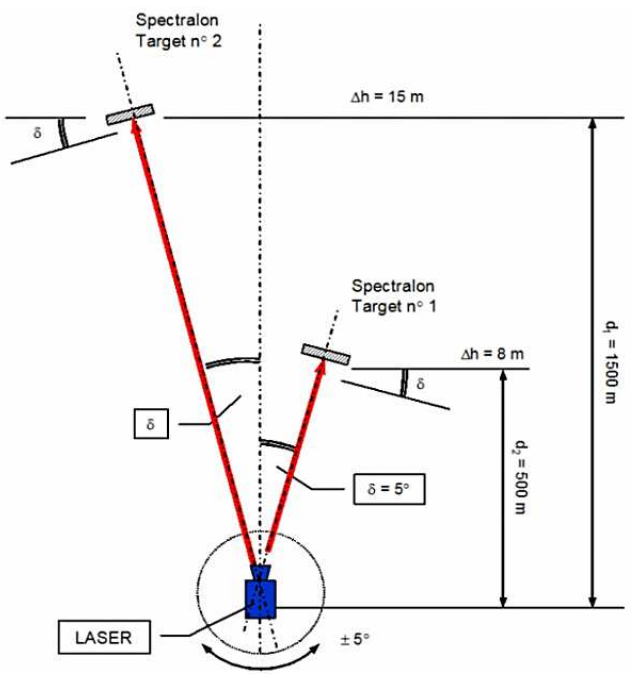

Fig. 63. Layout of a possible energy measurement technique for high PRF values.

The expression to determine atmospheric extinction $(\gamma)$ with this technique is:

$$
\gamma=\frac{1}{2 \Delta d} \cdot \ln \left[\left(\frac{V_{1}}{P_{O 1}} \cdot d_{1}^{2}\right) /\left(\frac{V_{2}}{P_{O 2}} \cdot d_{2}^{2}\right)\right]
$$

where:

$$
\begin{array}{ll}
\Delta d & =\mathrm{d}_{1}-\mathrm{d}_{2} \\
V_{1} & =\text { anodic voltage at receiver for the first target } \\
V_{2} & =\text { anodic voltage at receiver for the second target } \\
P_{O 1} / P_{O 2} & =\text { emitted laser powers }
\end{array}
$$

More details about the Energy Measurement Techniques are provided in [51, 150].

\subsubsection{Examples of experimental measurements}

Examples of test results including laser beam atmospheric propagation measurements, pointing accuracy, systems harmonisation and performance evaluation trials are presented below. A detailed discussion about the ground experimental activities performed at two different NIR laser wavelengths ( $\lambda=1064 \mathrm{~nm}$ and $\lambda=1540 \mathrm{~nm}$ ) to develop correction functions for the ESLM model is provided in [12, 52]. As a result of these activities a Laser Propagation Database (LPD) has been created and it is being progressively refined based data collected during a test and training missions with a variety of tactical laser systems in a comprehensive range of weather conditions. As an example of the aiming accuracy measurements, Fig. 64 shows results relative to the energy and geometric pointing errors of an A-LTD system. Fig. 65 shows the difference between the geometric and energy pointing errors.

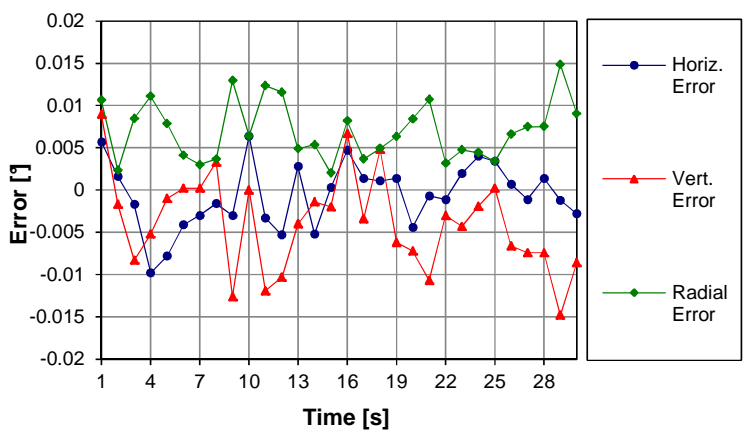

Fig. 64. A-LTD pointing accuracy.

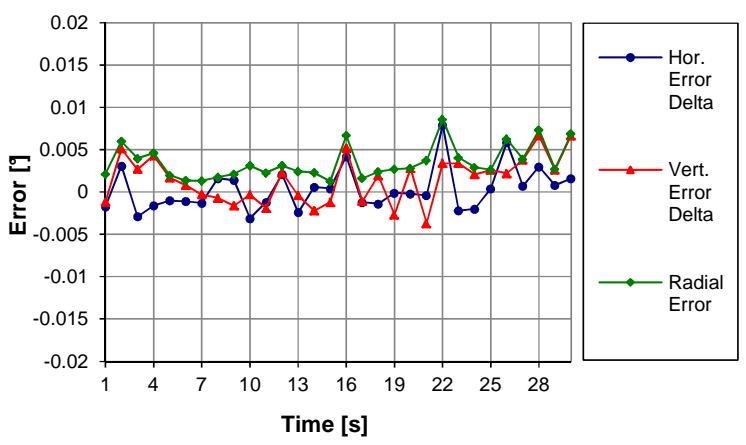


This is the author pre-publication version. This paper does not include the changes arising from the revision, formatting and publishing process. The final paper that should be used for referencing is:

R. Sabatini, M. A. Richardson, A. Gardi, S. Ramasamy, "Airborne laser sensors and integrated systems", Progress in Aerospace Sciences (2015), http: //dx.doi.org/10.1016/j.paerosci.2015.07.002i.

Fig. 65. Measured geometric vs. energy pointing errors.

Results of the spot spreading measurements (average $2 R_{I}$ values) for the A-LTD system are shown in Fig. 66, together with the calculated $1 / \mathrm{e}$ and $95 \%$-energy spot diameters. Although in certain cases the measured spot diameter (average of 150-200 measurements) was less that the calculated 95\%-energy spot diameter, the average data showed that the spot spreading was much more significant at greater slant-ranges.

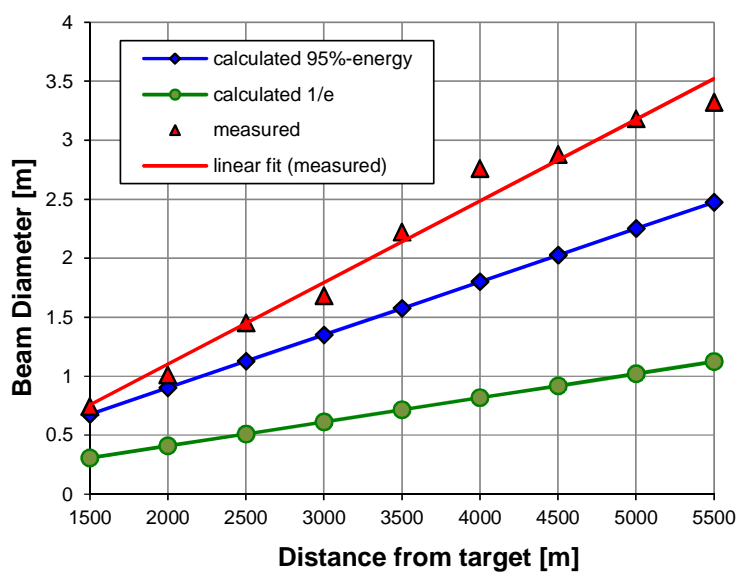

Fig. 66. A-LTD calculated and measured spot diameter as a function of slant-range for normal incidence.

Furthermore, it was observed that also the parameters increased significantly their values at increasing slat-ranges. The average spot quality values and their variations during measurements performed with the A-LTD $(\lambda=1064 \mathrm{~nm})$ at $\mathrm{SR}=1500 \mathrm{~m}, 3.5$ $\mathrm{km}$ and $5.5 \mathrm{~km}$ are listed in Table 10.

\section{Table 10}

Measured spot quality parameters.

\begin{tabular}{|c|c|c|c|c|c|c|}
\hline \multirow{2}{*}{$S P D$} & \multicolumn{2}{|c|}{$1500 \mathrm{~m}$} & \multicolumn{2}{c|}{$3.5 \mathrm{~km}$} & \multicolumn{2}{c|}{$5.5 \mathrm{~km}$} \\
\cline { 2 - 7 } & $\boldsymbol{\mu}$ & $\boldsymbol{\sigma}$ & $\boldsymbol{\mu}$ & $\boldsymbol{\sigma}$ & $\boldsymbol{\mu}$ & $\boldsymbol{\sigma}$ \\
\hline $\boldsymbol{Q}_{S}$ & 0.8455 & 0.1350 & 0.8381 & 0.1799 & 0.6860 & 0.2830 \\
\hline $\boldsymbol{Q}_{\boldsymbol{d e}}$ & 0.8329 & 0.0913 & 0.7184 & 0.1575 & 0.6119 & 0.1837 \\
\hline $\boldsymbol{Q}_{\boldsymbol{d g}}$ & 0.7275 & 0.1289 & 0.6930 & 0.1340 & 0.6607 & 0.1723 \\
\hline
\end{tabular}

With increasing slant-range all spot quality parameters were characterised by a progressive reduction of their mean values and greater dispersions. Obviously, as all spot quality parameters vary between 0 and 1. Fig. 67 shows the mission profile of an airborne laser system. The various dive manoeuvres are depicted, with grazing angles between $20^{\circ}$ and $50^{\circ}$. The illuminated target is positioned in $(0,0)$. The red line depicts the lower limit for the safe activation of the laser system, to guarantee eye-safety.

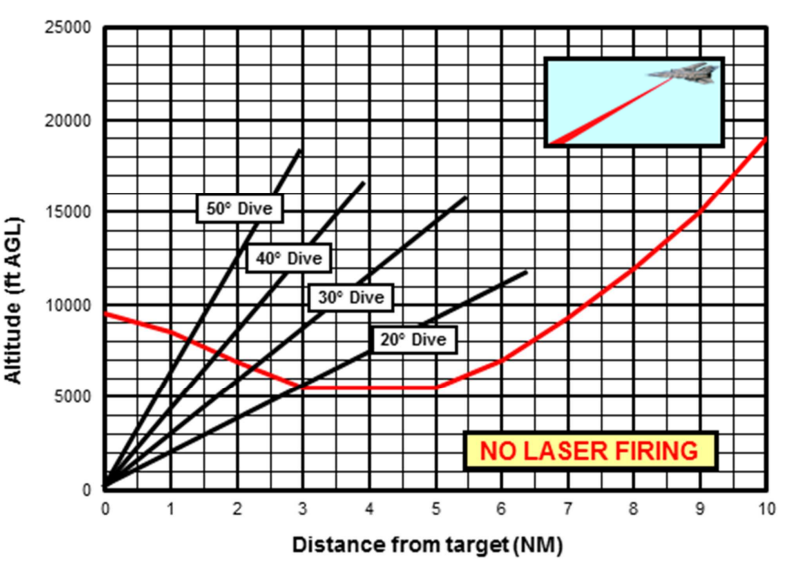

Fig. 67. Example flight test mission profiles.

Experimental data collected during the manoeuvres depicted in Fig. 67 are represented in Fig. 68. In particular, the variation between the extinction coefficient $\left(\gamma_{\text {atm }}{ }^{H}\right)$ and the one relative to the mean sea level $\left(\gamma_{a t m}\right)$ are depicted as a function of altitude and grazing angle.

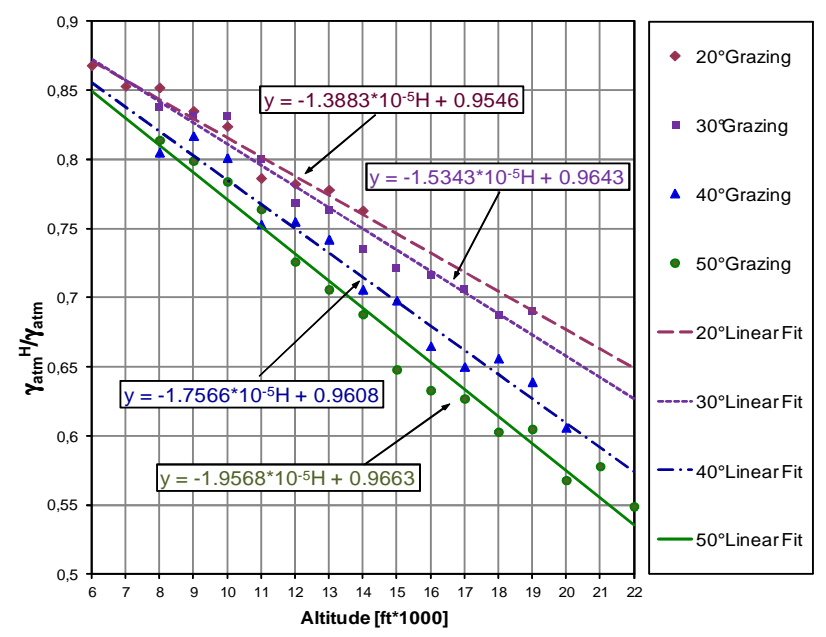

Fig. 68. $\gamma_{\mathrm{atmH}} / \gamma_{\mathrm{atm}}$ relationship as a function of altitude and grazing angle.

\section{Conclusions}

A A review on state-of-the-art airborne laser technologies and innovative concepts for Research, Development, Test and Evaluation (RDT\&E) in this field were presented. To provide a coherent framework for civil/military systems design and for test and evaluation activities, a detailed discussion of airborne lasers performance analysis was presented. Additionally, the methods and algorithms required for the evaluation of laser systems eyesafety criteria in various representative scenarios were presented. Finally, useful guidelines for laboratory, ground and flight test activities for airborne laser systems were elaborated. Based on extensive experience with RDT\&E of airborne laser systems, a tailored development-test philosophy has been presented that reduces the costs associated with the process and achieves the highest possible level of efficiency. Particularly, instead of carrying out flight trials at the end of the systems design and integration process, there is a constant involvement of flight test human resources in the various design/integration phases and participation of design/integration engineers to the flight test 
This is the author pre-publication version. This paper does not include the changes arising from the revision, formatting and publishing process. The final paper that should be used for referencing is:

R. Sabatini, M. A. Richardson, A. Gardi, S. Ramasamy, "Airborne laser sensors and integrated systems", Progress in Aerospace Sciences (2015), http: //dx.doi.org/10.1016/j.paerosci.2015.07.002i.

planning activities. Once the electrical/mechanical compatibility of laser sub-systems with aircraft is assured, the kernel areas of testing of aerodynamics, flight mechanics, software, hardware and store separation (as applicable) are addressed. Flight test requirements, performance models and analysis tools provide guidelines for defining the aircraft Flight Test Instrumentation (FTI) data acquisition and telemetry system requirements. Simulation is essential for correctly planning flight test activities, analysing flight test data and verifying the validity of the models/algorithms loaded in the operational avionics software. Particularly, use of suitable simulation tools allows a full aerodynamics and safe-separation investigation, weapon aiming analysis, masking characterisation, preliminary performance estimation, laser hazards determination and laser/ballistic safety assessment, with consequent significant speed-up in the systems integration activities. This balanced development/test methodology, which consisted in a continuous interaction between ground test, flight test and simulation, provides significant benefits and introduces improvements in the process efficiency (development and test activity speed-up), contributing to the optimisation of flight test data gathering and to a remarkable reduction of verification and validation costs.

\section{References}

1. Hecht, J., A short history of laser development. Applied Optics, 2010. 49(25): p. F99-F122.

2. Jebur, M.N., B. Pradhan, and M.S. Tehrany, Optimization of landslide conditioning factors using very high-resolution airborne laser scanning (LiDAR) data at catchment scale. Remote Sensing of Environment, 2014. 152(0): p. 150-165.

3. Thomas Nugent, J., DJordin Kare, David Bashford, Carsten Erickson, and Jeff Alexander, 12-HOUR HOVER: FLIGHT DEMONSTRATION OF A LASER POWERED QUADROCOPTER, in AUVSI-white-paper-8-11.

4. Ackermann, F., Airborne laser scanning - present status and future expectations. ISPRS Journal of Photogrammetry and Remote Sensing, 1999. 54(2): p. 64-67.

5. Baltsavias, E.P., A comparison between photogrammetry and laser scanning. ISPRS Journal of photogrammetry and Remote Sensing, 1999. 54(2): p. 83-94.

6. N. D. Quadros, P.A.C., C.S. Fraser, Integration of bathymetric and topographic Lidar: a preliminary investigation. Remote Sensing and Spatial Information Sciences 37: p. 1299-1304.

7. Penny, M., B. Billard, and R. Abbot, LADS-The Australian laser airborne depth sounder. International Journal of Remote Sensing, 1989. 10(9): p. 1463-1479.

8. S. Purkis, J.B., in Coral Reef Remote Sensing, S.J.P. J. A. Goodman, S. R. Phinn, Editor. 2013, Springer Netherlands. p. 115-143.

9. Tuell, G.H., et al. Measurement of ocean water optical properties and seafloor reflectance with Scanning Hydrographic Operational Airborne Lidar Survey (SHOALS): II. Practical results and comparison with independent data. in Optics \& Photonics 2005. 2005. International Society for Optics and Photonics.

10. Tuell, G.H. and J.Y. Park. Use of SHOALS bottom reflectance images to constrain the inversion of a hyperspectral radiative transfer model. in Defense and Security. 2004. International Society for Optics and Photonics.

11. Kamerman, G., Laser Radar EM]. Chapter 1 of Active ElectroOptical System. Vo1. 6. The Infrared and Electro-Optical System Handbook. 1993, SPIE Press.

12. Sabatini, R. and M.A. Richardson, Airborne Laser Systems Testing and Analysis, RTO AGARDograph AG-300 Vol. 26. Systems Concepts and Integration Panel (SCI-126). 2010: NATO Science and Technology Organization.

13. Arras, K.O., N. Tomatis, and R. Siegwart. Multisensor on-the-fly localization using laser and vision. in Intelligent Robots and Systems, 2000.(IROS 2000). Proceedings. 2000 IEEE/RSJ International Conference on. 2000. IEEE.

14. Powell, G., et al. Data fusion of FLIR and LADAR in autonomous weapons systems. in Proceedings of Sixth International Conference on Information Fusion (Fusion 2003) Cairns, Australia. 2003.
15. Johnson, E.N., et al., Active-Vision Control Systems For Complex Adversarial 3-D Environments. 2009, DTIC Document.

16. Asner, G.P., et al., Carnegie airborne observatory: in-flight fusion of hyperspectral imaging and waveform light detection and ranging for three-dimensional studies of ecosystems. Journal of Applied Remote Sensing, 2007. 1(1): p. 013536-013536-21.

17. Lefsky, M.A., et al., Lidar remote sensing of above-ground biomass in three biomes. Global Ecology and Biogeography, 2002. 11(5): p. 393399.

18. Commission, F. Radar and Lidar, Lidar Applications. Available from: http://www.forestry.gov.uk/forestry/infd-6rvc5a.

19. Andersen, H.-E., R.J. McGaughey, and S.E. Reutebuch, Estimating forest canopy fuel parameters using LIDAR data. Remote sensing of Environment, 2005. 94(4): p. 441-449.

20. Sittler, B., Revealing historical landscapes by using airborne laser scanning. Laser-Scanners for Forest and Landscape Assessment, 2004.

21. Henning, J.G. and P.J. Radtke, Ground-based laser imaging for assessing three-dimensional forest canopy structure. Photogrammetric Engineering \& Remote Sensing, 2006. 72(12): p. 1349-1358.

22. Lee, A., R. Lucas, and C. Brack, Quantifying vertical forest stand structure using small footprint lidar to assess potential stand dynamics. Proceedings, NATSCAN-Laser scanners for forest and landscape assessment instruments, processing methods and applications, 2004: p. 3-6.

23. Nelson, R., R. Oderwald, and T.G. Gregoire, Separating the ground and airborne laser sampling phases to estimate tropical forest basal area, volume, and biomass. Remote Sensing of Environment, 1997. 60(3): p. 311-326.

24. Holmgren, J. and T. Jonsson, Large scale airborne laser scanning of forest resources in Sweden. International Archives of photogrammetry, remote sensing and spatial information sciences, 2004. 36(Part 8): p. W2.

25. Reutebuch, S.E., H.-E. Andersen, and R.J. McGaughey, Light detection and ranging (LIDAR): an emerging tool for multiple resource inventory. Journal of Forestry, 2005. 103(6): p. 286-292.

26. Carter, W.E., An interlude with Dirac. Am. Sci, 2012. 100: p. 438-41.

27. Williams, J.G., S.G. Turyshev, and D.H. Boggs, Progress in lunar laser ranging tests of relativistic gravity. Physical Review Letters, 2004. 93(26): p. 261101

28. McGarry, J. and T. Zagwodzki, A brief history of satellite laser ranging: 1964-present. Published by the Crustal Dynamics Data Information System (CDDIS), NASA Goddard Space Flight Center, Greenbelt, Maryland, 2005.

29. Sabatini, R., E. Roviaro, and M. Cottalasso, Development of a Laser Collision Avoidance System for Helicopters: Obstacle Detection/Classification and Calculation of Alternative Flight Paths, in RTO-MP-092 - Complementarity of Ladar and Radar. 2002, NATO Research and Technology Organization (RTO).

30. Sabatini, R., A. Gardi, and M.A. Richardson, LIDAR Obstacle Warning and Avoidance System for Unmanned Aircraft. International Journal of Mechanical, Aerospace, Industrial and Mechatronics Engineering, 2014. 8: p. 62-73.

31. Sabatini, R., A. Gardi, and S. Ramasamy. A Laser Obstacle Warning and Avoidance System for Manned and Unmanned Aircraft. in IEEE Metrology for Aerospace (MetroAeroSpace 2014). 2014. Benevento, Italy: IEEE.

32. Sabatini, R., A. Gardi, and S. Ramasamy, A Laser Obstacle Warning and Avoidance System for Unmanned Aircraft Sense-and-Avoid. Applied Mechanics and Materials, 2014. 629: p. 355-360.

33. Fleck Jr, J.A., J.R. Morris, and M.D. Feit, Time-dependent propagation of high energy laser beams through the atmosphere. Applied Physics, 1976. 10(2): p. 129-160.

34. Gebhardt, F.G., High Power Laser Propagation. Applied Optics, 1976. 15(6): p. 1479-1493.

35. Müller, D., U. Wandinger, and A. Ansmann, Microphysical particle parameters from extinction and backscatter lidar data by inversion with regularization: theory. Applied Optics, 1999. 38(12): p. 23462357.

36. Veselovskii, I., et al., Inversion of multiwavelength Raman lidar data for retrieval of bimodal aerosol size distribution. Applied Optics, 2004. 43(5): p. 1180-1195.

37. Müller, D., et al., Microphysical particle parameters from extinction and backscatter lidar data by inversion with regularization: Experiment. Applied Optics, 2000. 39(12): p. 1879-1892. 
This is the author pre-publication version. This paper does not include the changes arising from the revision, formatting and publishing process. The final paper that should be used for referencing is:

R. Sabatini, M. A. Richardson, A. Gardi, S. Ramasamy, "Airborne laser sensors and integrated systems", Progress in Aerospace Sciences (2015), http: //dx.doi.org/10.1016/j.paerosci.2015.07.002i.

38. Kuang, Z., et al., Spaceborne measurements of atmospheric CO2 by high-resolution NIR spectrometry of reflected sunlight: An introductory study. Geophysical Research Letters, 2002. 29(15): p. 11-1 to $11-4$.

39. Dufour, E. and F.M. Bréon, Spaceborne estimate of atmospheric CO2 column by use of the differential absorption method: Error analysis. Applied Optics, 2003. 42(18): p. 3595-3609.

40. Krainak, M.A., et al. Measurements of atmospheric CO2 over a horizontal path using a tunable-diode-laser and erbium-fiberamplifier at $1572 \mathrm{~nm}$. in Conference on Lasers and Electro-Optics 2003 (CLEO '03). 2003. Baltimore, MD, USA: IEEE / Optical Society of America.

41. Riris, H., et al. A laser sounder for measuring atmospheric trace gases from space. in SPIE 6750, Lidar Technologies, Techniques, and Measurements for Atmospheric Remote Sensing III. 2007. Florence, Italy.

42. Allan, G.R., et al. Laser sounder for active remote sensing measurements of CO2 concentrations. in IEEE/AIAA Aerospace Conference 2008 (AC2008). 2008. Big Sky, MT, USA: IEEE.

43. Amediek, A., et al., Airborne lidar reflectance measurements at 1.57 $\mu \mathrm{m}$ in support of the A-SCOPE mission for atmospheric CO2. Atmospheric Measurement Techniques Discussions, 2009. 2(3): p. 1487-1536.

44. Caron, J.R. and Y. Durand, Operating wavelengths optimization for a spaceborne lidar measuring atmospheric co2. Applied Optics, 2009. 48(28): p. 5413-5422.

45. Abshire, J.B., et al., Pulsed airborne lidar measurements of atmospheric CO2 column absorption. Tellus, Series B: Chemical and Physical Meteorology, 2010. 62(5): p. 770-783.

46. Choi, S., et al., Implementation of differential absorption LIDAR (DIAL) for molecular iodine measurements using injection-seeded laser. Journal of the Optical Society of Korea, 2012. 16(4): p. 325330.

47. Abshire, J.B., et al., Airborne measurements of $\mathrm{CO} 2$ column concentration and range using a pulsed direct-detection IPDA lidar. Remote Sensing, 2013. 6(1): p. 443-469.

48. Abshire, J.B., et al., Airborne measurements of $\mathrm{CO} 2$ column absorption and range using a pulsed direct-detection integrated path differential absorption lidar. Applied Optics, 2013. 52(19): p. 44464461.

49. Pelon, J., et al., LIDAR and RADAR Observations, in Airborne Measurements for Environmental Research: Methods and Instruments. 2013, Wiley-VCH. p. 457-526.

50. Sabatini, R. and M.A. Richardson. Innovative methods for planetary atmospheric sounding by lasers. in AIAA Space 2008 Conference. 2008. San Diego, CA, USA.

51. Sabatini, R., et al. Airborne laser systems for atmospheric sounding in the near infrared. in SPIE 8433, Laser Sources and Applications, Photonics Europe 2012. 2012. Brussels, Belgium.

52. Sabatini, R. and M.A. Richardson, Novel atmospheric extinction measurement techniques for aerospace laser system applications. Infrared Physics and Technology, 2013. 56: p. 30-50.

53. Gardi, A., R. Sabatini, and S. Ramasamy, Bistatic LIDAR System for the Characterisation of Aviation-Related Pollutant Column Densities. Applied Mechanics and Materials, 2014. 629: p. 257-262.

54. Gardi, A., R. Sabatini, and G. Wild. Unmanned aircraft bistatic lidar for CO2 colum density determination. in IEEE Metrology for Aerospace (MetroAeroSpace) 2014. 2014. Benevento, Italy: IEEE.

55. Sabatini, R. Innovative Flight Test Instrumentation and Techniques for Airborne Laser Systems Performance Analysis and Mission Effectiveness Evaluation. in IEEE Metrology for Aerospace 2014 (MetroAeroSpace 2014). 2014. Benevento, Italy: IEEE.

56. Heard, D.E., ed. Analytical Techniques for Atmospheric Measurement. 2006, Blackwell Publishing: Oxford, UK.

57. Scott, N.A., et al., Characteristics of the TOVS pathfinder path-B dataset. Bulletin of the American Meteorological Society, 1999. 80(12): p. 2679-2701.

58. Bartlett, L.M. and A.R. Webb, Changes in ultraviolet radiation in the 1990s: Spectral measurements from Reading, England. Journal of Geophysical Research: Atmospheres, 2000. 105(D4): p. 4889-4893.

59. Kormann, R., et al., Application of a multi-laser tunable diode laser absorption spectrometer for atmospheric trace gas measurements at sub-ppbv levels. Spectrochimica Acta - Part A Molecular and Biomolecular Spectroscopy, 2002. 58(11): p. 2489-2498.

60. Fischer, H., et al., Synoptic tracer gradients in the upper troposphere over central Canada during the Stratosphere-Troposphere experiments by Aircraft Measurements 1998 summer campaign. Journal of Geophysical Research D: Atmospheres, 2002. 107(7-8): p. $5-1$.

61. De Reus, M., et al., On the relationship between acetone and carbon monoxide in different air masses. Atmospheric Chemistry and Physics, 2003. 3(5): p. 1709-1723.

62. Wert, B.P., et al., Design and performance of a tunable diode laser absorption spectrometer for airborne formaldehyde measurements. Journal of Geophysical Research: Atmospheres, 2003. 108(12): p. ACH 1-1 - ACH 1-15.

63. Viciani, S., et al., A cryogenically operated laser diode spectrometer for airborne measurement of stratospheric trace gases. Applied Physics B: Lasers and Optics, 2008. 90(3-4): p. 581-592.

64. D'Amato, F., P. Mazzinghi, and F. Castagnoli, Methane analyzer based on TDL's for measurements in the lower stratosphere: Design and laboratory tests. Applied Physics B: Lasers and Optics, 2002. 75(23): p. 195-202.

65. Zondlo, M.A., et al., Development and characterization of an airborne-based instrument used to measure nitric acid during the NASA Transport and Chemical Evolution over the Pacific field experiment. Journal of Geophysical Research D: Atmospheres, 2003. 108(20): p. GTE 14-1 - 14-18.

66. Durry, G., et al., Laser diode spectroscopy of H2O at $2.63 \mu \mathrm{m}$ for atmospheric applications. Applied Physics B: Lasers and Optics, 2008. 90(3-4): p. 573-580.

67. Kerstel, E. and L. Gianfrani, Advances in laser-based isotope ratio measurements: Selected applications. Applied Physics B: Lasers and Optics, 2008. 92(3 SPECIAL ISSUE): p. 439-449.

68. Dyroff, C., D. Fütterer, and A. Zahn, Compact diode-laser spectrometer ISOWAT for highly sensitive airborne measurements of water-isotope ratios. Applied Physics B: Lasers and Optics, 2010. 98(2-3): p. 537-548.

69. McQuaid, J., et al., In Situ Trace Gas Measurements, in Airborne Measurements for Environmental Research: Methods and Instruments. 2013, Wiley-VCH. p. 77-155.

70. Beck, M., et al., Continuous wave operation of a mid-infrared semiconductor laser at room temperature. Science, 2002. 295(5553): p. 301-305.

71. Werle, P., et al., Near- and mid-infrared laser-optical sensors for gas analysis. Optics and Lasers in Engineering, 2002. 37(2-3): p. 101114.

72. Cathabard, O., et al., Quantum cascade lasers emitting near $2.6 \mu \mathrm{m}$. Applied Physics Letters, 2010. 96(14).

73. Li, J.S., W. Chen, and H. Fischer, Quantum cascade laser spectrometry techniques: A new trend in atmospheric chemistry. Applied Spectroscopy Reviews, 2013. 48(7): p. 523-559.

74. Herndon, S.C., et al., Airborne measurements of $\mathrm{HCHO}$ and $\mathrm{HCOOH}$ during the New England Air Quality Study 2004 using a pulsed quantum cascade laser spectrometer. Journal of Geophysical Research: Atmospheres, 2007. 112(10).

75. Kort, E.A., et al., Tropospheric distribution and variability of N2O: Evidence for strong tropical emissions. Geophysical Research Letters, 2011. 38(15).

76. Wunch, D., et al., Calibration of the total carbon column observing network using aircraft profile data. Atmospheric Measurement Techniques, 2010. 3(5): p. 1351-1362.

77. Klippel, T., et al., Distribution of hydrogen peroxide and formaldehyde over Central Europe during the HOOVER project. Atmospheric Chemistry and Physics, 2011. 11(9): p. 4391-4410.

78. Wecht, K.J., et al., Validation of TES methane with HIPPO aircraft observations: Implications for inverse modeling of methane sources. Atmospheric Chemistry and Physics, 2012. 12(4): p. 1823-1832.

79. Santoni, G.W., et al., Evaluation of the airborne quantum cascade laser spectrometer (QCLS) measurements of the carbon and greenhouse gas suite-CO2, CH4, N2O, and CO-during the CalNex and HIPPO campaigns. Atmospheric Measurement Techniques, 2014. 7: p. $1509-1526$.

80. Brenguier, J.L., et al., In Situ Measurements of Cloud and Precipitation Particles, in Airborne Measurements for Environmental Research: Methods and Instruments. 2013, Wiley-VCH. p. 225-301.

81. Grossman, J., The Airborne Laser Laboratory (ALL) Completes Successful Tests, in International Defense Review. 1983.

82. Drain, L.E., The laser Doppler technique J.Wiley, New York, 1980.

83. Byun, G., Olcmen, M. S., Simpson, R. L., A Miniature Laser-Doppler Velocimeter for Simultaneous Three-Velocity-Component 
This is the author pre-publication version. This paper does not include the changes arising from the revision, formatting and publishing process. The final paper that should be used for referencing is:

R. Sabatini, M. A. Richardson, A. Gardi, S. Ramasamy, "Airborne laser sensors and integrated systems", Progress in Aerospace Sciences (2015), http: //dx.doi.org/10.1016/j.paerosci.2015.07.002i.

Measurements. Measurement Science and Technology, 2004. 15(10): p. 2075-2082.

84. Shapley, N.C., R.C. Armstrong, and R.A. Brown, Laser Doppler velocimetry measurements of particle velocity fluctuations in a concentrated suspension. Journal of Rheology, 2002. 46(1): p. 241272.

85. Coren, F. and P. Sterzai, Radiometric correction in laser scanning. International Journal of Remote Sensing, 2006. 27(15): p. 30973104.

86. Holmgren, J. and Å. Persson, Identifying species of individual trees using airborne laser scanner. Remote Sensing of Environment, 2004. 90(4): p. 415-423.

87. Wehr, A. and U. Lohr, Airborne laser scanning — an introduction and overview. ISPRS Journal of Photogrammetry and Remote Sensing, 1999. 54(2): p. 68-82.

88. Cramer, M. and N. Haala. Direct exterior orientation of airborne sensors-an accuracy investigation of an integrated GPS/inertial system. in Proc. ISPRS Workshop Comm. III/1', Portland, Maine, USA. 1999.

89. Treitz, P.M. and P.J. Howarth, Hyperspectral remote sensing for estimating biophysical parameters of forest ecosystems. Progress in Physical Geography, 1999. 23(3): p. 359-390.

90. Paolo Sterzai, M.V., Matteo Berti, Franco Coren, Alessandro Corsini, Alberto Rosi, Paolo Mora, Franco Zambonelli. Innovative integrated airborne and wireless systems for landslide monitoring. in Geoinformation for Disaster management (Gi4DM) 2010. 2010. Turin, Italy.

91. Mobley, C., et al., Optical modeling of ocean waters: Is the Case 1Case 2 classification still useful? Oceanography-Washington DCOceanography Society-, 2004. 17(2): p. 60-67.

92. Guenther, G.C., M.W. Brooks, and P.E. LaRocque, New capabilities of the "SHOALS" airborne lidar bathymeter. Remote Sensing of Environment, 2000. 73(2): p. 247-255.

93. Guenther, G.C., et al., Meeting the accuracy challenge in airborne lidar bathymetry. EARSel, Dresden, 2000.

94. Doneus, M., et al., Airborne laser bathymetry-detecting and recording submerged archaeological sites from the air. Journal of Archaeological Science, 2013. 40(4): p. 2136-2151.

95. Brock, J.C. and S.J. Purkis, The emerging role of lidar remote sensing in coastal research and resource management. Journal of Coastal Research, 2009: p. 1-5.

96. Vierling, K.T., et al., Lidar: shedding new light on habitat characterization and modeling. Frontiers in Ecology and the Environment, 2008. 6(2): p. 90-98.

97. Kevin, J. S., Parent, A., Levesque, M., Galarneau, P. Active range-gated near-IR TV system for all-weather surveillance. Proc. SPIE 2935, Surveillance and Assessment Technologies for Law Enforcement, 171, Feb 1997. doi:10.1117/12.266798.

98. Alastair N. J., Vandenberg, M., Tritchew, S., Bonnier, D., Larochelle, V. ALBEDOS: an airborne laser-based enhanced detection and observation system for coast guard and maritime patrol application. Proc. SPIE 2269, Infrared Technology, 201 (October 17, 1994); doi:10.1117/12.188637.

99. Sohn, G, and Dowman. I. Data fusion of high-resolution satellite imagery and LiDAR data for automatic building extraction, ISPRS Journal of Photogrammetry and Remote Sensing, vol. 62, issue 1, p. 43-63. 2007.

100. Rottensteiner, F. Automatic generation of high-quality building models from lidar data. IEEE Computer Graphics and Applications, vol. 23, issue 6, pp. 42-50, 2003.

101. Rottensteiner, F., and Briese. C. A new method for building extraction in urban areas from high-resolution LIDAR data. International Archives of Photogrammetry Remote Sensing and Spatial Information Sciences 34.3/A, p. 295-301, 2002.

102. Liang, C. et al. Integration of LiDAR data and optical multi-view images for $3 D$ reconstruction of building roofs. Optics and Lasers in Engineering, vol. 51, issue 4, p. 493-502, 2013.

103. Kasser, J. S.S. The United States Airborne Laser Test Program: A Case Study. in SETE2013. 2013. Canberra, Australia.

104. R.J., G.R.A.F., Development of a Wideband Airborne Laser Data Link. AGARD-CP-595, 1998.

105. J. Shwartz, G.T.W., J. Avidor, Tactical High Energy Laser, in SPIE, Vol. 4632 - Laser and Beam Control Technologies. 2002: Bellingham, WA.

106. J.P.Dudeja, G.S.K., Shipborne Laser Beam Weapon System for Defence against Cruise Missiles. Defence Science Journal, 2000. 50(2): p. 231-240.
107. Kopp, C., High Energy Laser Directed Energy Weapons, in Technical Report APA-TR-2008-0501, Defense Today. April 2002.

108. Lamberson, S., H. Schall, and P. Shattuck. The airborne laser. 2006.

109. Kasser, J. and S. Sen, The United States Airborne Laser Test Bed program: A case study.

110. Anil Kumar Maini, A.L.V., Varsha Agrawal, Target Simulator for Serviceability Check of Infrared-Guided Missiles. Journal of Spacecraft and Rockets, 2011. 48(1): p. 175-181.

111. deLeon, P., The Laser-Guided Bomb: Case History of a Development. Rand Corporation, Santa Monica, CA, 1974.

112. J.West, W. Developmental Testing of A Laser-Guided Bomb Simulation. in U.S. Air Force T\&E Days. 2008. Los Angeles, California, USA.

113. R. Sabatini, F.G., G. Campo, A. Marciante, Laser Guided Bombs and Convertible Designation Pod Integration on Italian TORNADO-IDS, in 31st Annual Symposium of the Society of Flight Test Engineers "Flight Test in the New Millennium" 2000: Turin, Italy.

114. R. Sabatini, F.G., G. Campo, A. Marciante. Simulation and Flight Testing for Integration of a Laser Designation Pod and Laser Guided Bombs on Italian TORNADO-IDS. in Symposium "Integration of Simulation with System Testing" 2001. Toulouse, France: NATO-RTO Systems Concepts and Integration (SCI) Panel.

115. R. Sabatini, M.A.R., System Integration and Flight Testing of a Laser Designation Pod and Laser Guided Bombs on the Italian TORNADO Interdiction and Strike Aircraft. Journal of Battlefield Technology, 2001. 4(2): p. 37-48.

116. Ralph, J.F., Edwards, K.L., . The Effect of Aircraft Biases on the Delivery of an Enhanced Laser Guided Weapon. in ICAS 2002. 2004. Southwest Research Institute.

117. Corps, U.S.M., Fire Support Coordination in the Ground Combat Element, in Marine Corps Warfighting Publication 3-16. Quantico, Virginia: Marine Corps Combat Development Center, Doctrine Command. 2001.

118. Lele, A., Speed of Light Weaponry: Directed Energy Weapons. Strategic Technologies for the Military - Breaking New Frontiers. 2010: SAGE Publications.

119. Sabatini, R. and M.A. Richardson, System Integration and Flight Testing of a Laser Designation Pod and Laser Guided Bombs on the Italian TORNADO Interdiction and Strike Aircraft. Journal of Battlefield Technology, 2001. 4(2): p. 37-48.

120. Sabatini, R. and M.A. Richardson, Airborne Laser Systems Testing, Safety Analysis, Modelling and Simulation, in 21st Annual Symposium of the Society of Flight Test Engineers - European Chapter. 2010: Vergiate (VA), Italy.

121. Johnson, P. Unmanned Aerial Vehicle as the platform for lightweight laser sensing to produce sub-meter accuracy terrain maps for less than $\$ 5 / \mathrm{km}^{2}$. Mech. Eng. Dept., Columbia Univ., New York, 2006.

122. Nagai, M., Chen, T., Shibasaki, R., Kumagai, H. and Ahmed, A. UAV borne 3-d mapping system by multisensor integration. IEEE Trans. Geosci. Remote Sens., vol. 47, no. 3, pp. 701-708, Mar. 2009.

123. Zhao, X., Liu, J. and Tan, M. A remote aerial robot for topographic survey. in Proc. IEEE Int. Conf. Intell. Robots Syst., 2006, p. 31433148.

124. Geske, J., MacDougal, M., Stahl, R. and Snyder, D.R. Miniature laser rangefinders and laser altimeters. in Proc. IEEE Conf. Avionics FiberOpt. Photon. Technol., 2008, p. 53-54.

125. Lambers, K., Eisenbeiss, H., Sauerbier, M., Kupferschmidt, D., Gaisecker, T., Sotoodeh, S. and Hanusch, T. Combining photogrammetry and laser scanning for the recording and modeling of the late intermediate period site of Pinchango Alto. Palpa, Peru," J. Archaeol. Sci., vol. 34, no. 10, p. 1702-1712, Oct. 2007.

126. Yu, X., Hyyppä, J., Kaartinen, H. and Maltamo, M. Automatic detection of harvested trees and determination of forest growth using airborne laser scanning. Remote Sens. Environ., vol. 90, no. 4, pp. 451-462, April 2004.

127. Lin, Y., Hyyppa, J., and Jaakkola, A. mini-UAV-borne LIDAR for finescale mapping. Geoscience and Remote Sensing Letters, IEEE, vol. 8, no. 3, pp. 426-430, 2011.

128. Wallace, L., Lucieer, A., Watson, C., Turner, D. Development of a UAVLiDAR system with application to forest inventory. Remote Sens 2012, 4, 1519-1543.

129. Jaakkola, A., Hyyppä, J., Kukko, A., Yu, X., Kaartinen, H., Lehtomäki, M., Lin, Y. A low-cost multi-sensoral mobile mapping system and its feasibility for tree measurements. ISPRS J. Photogramm 2010, 65, p. 514-522. 
This is the author pre-publication version. This paper does not include the changes arising from the revision, formatting and publishing process. The final paper that should be used for referencing is:

R. Sabatini, M. A. Richardson, A. Gardi, S. Ramasamy, "Airborne laser sensors and integrated systems", Progress in Aerospace Sciences (2015), http: //dx.doi.org/10.1016/j.paerosci.2015.07.002i.

130. Lin, Y., Hyyppä, J. and Jaakkola, A. Mini-UAV-borne LIDAR for finescale mapping. IEEE Geosci. Remote Sens. Lett 2011, 8, 426-430.

131. Campbell, J., De Haag, M.U. and Graas, F.V. Terrain-referenced precision approach guidance: Proof-of-concept flight test results. Navigation, vol. 54, pp. 21-29, 2007.

132. Chisholm, R.A., et al., UAV LiDAR for below-canopy forest surveys. Journal of Unmanned Vehicle Systems, 2013. 1(01): p. 61-68.

133. Eisenbeiss, H. and L. Zhang, Comparison of DSMs generated from mini UAV imagery and terrestrial laser scanner in a cultural heritage application. International Archives of Photogrammetry, Remote Sensing and Spatial Information Sciences XXXVI-5, 90e96, 2006.

134. Hebel, M. and U. Stilla, LiDAR-supported navigation of UAVs over urban areas. Surveying and Land Information Science, 2010. 70(3): p. 139-149.

135. Wallace, L., et al., Development of a UAV-LiDAR system with application to forest inventory. Remote Sensing, 2012. 4(6): p. 15191543.

136. Feldmann, R.J. and R.A. Gill. Development of laser crosslink for airborne operations. in Military Communications Conference, 1998. MILCOM 98. Proceedings., IEEE. 1998. IEEE.

137. Feldmann, R.J. and S.K. Rogers. Airborne laser communications scintillation measurements and model: A comparison of results. in 1988 Los Angeles Symposium--OE/LASE'88. 1988. International Society for Optics and Photonics.

138. Louthain, J.A., Anisoplanatic Approach to Airborne Laser Communication. in Meeting of the Military Sensing Symposia (MSS) Specialty Group on Active E-O Systems I(AD02), . 2007.

139. Crabtree, P.N., Performance-Metric Driven Atmospheric Compensation for Robust Free-Space Laser Communication, in PhD. 2006, Air Force Institute of Technology, Wright-Patterson AFB: OH.

140. Tyson, R.K., Adaptive optics and ground-to-space laser communications. Applied optics, 1996. 35(19): p. 3640-3646.

141. Andrews, L.C. and R.L. Phillips, Laser beam propagation through random media. Vol. 10. 2005: SPIE press Bellingham.

142. Louthain, J.A. and J.D. Schmidt. Integrated approach to airborne laser communication. in SPIE Remote Sensing. 2008. International Society for Optics and Photonics.

143. Kazemi, A.A. Intersatellite laser communication systems for harsh environment of space. in SPIE Defense, Security, and Sensing. 2013. International Society for Optics and Photonics.

144. Kazemi, A.A. and Panahi, A., Space-based laser systems for intersatellite communications. in SPIE Defense, Security, and Sensing. 2012. International Society for Optics and Photonics.

145. Shantha Lakshmi, K., Senthil Kumar, M. and Kavitha, K. Intersatellite laser communication system. in Computer and Communication Engineering, 2008. ICCCE 2008. International Conference on. 2008. IEEE.

146. Toyoshima, M. and Y. Takayama. Space-Based Laser Communication Systems and Future Trends. in CLEO: Applications and Technology. 2012. Optical Society of America.

147. Zhou, L. Optical System in Laser Inter-Satellites Communication. in Advanced Materials Research. 2014. Trans Tech Publ.

148. Toyoshima, M., et al., Ground-to-satellite laser communication experiments. Aerospace and Electronic Systems Magazine, IEEE, 2008. 23(8): p. 10-18.

149. Skolnik, M.I., Introduction to radar. Radar Handbook. 1962. 2.

150. Jelalian, A.V., Laser radar systems. 1992: Artech House.

151. D.D., T.M.E.a.D., Atmospheric Transmission, in ERIM-SPIE IR\&EO Systems Handbook 1996.

152. Weichel, H., Laser beam propagation in the atmosphere. Vol. 3. 1990: SPIE press.

153. Keith G.G., O.L.J., Rose W.C., Aerodynamic Effects, in ERIM-SPIE IR\&EO Systems Handbook 1996.

154. al., S.J.W.e., Laser Beam Propagation in the Atmosphere. Topics in Applied Physics Series, Springer-Verlag 1978. 25.

155. Gilbert, K.G. and L.J. Otten, Aero-optical phenomena. Progress in Astronautics and Aeronautics, 1982. 80.

156. Thomas, M.E., Optical Propagation in Linear Media: Atmospheric gases and particles, solid-state components, and water. Optical Propagation in Linear Media: Atmospheric gases and particles, solid-state components, and water, by Michael E. Thomas. ISBN-10 0-19-509161-2; ISBN-13 978-0-19-509161-8. Published by Oxford University Press, Oxford, UK, 2006., 2006. 1.

157. Jacquinet-Husson, N., et al., The 1997 spectroscopic GEISA databank. Journal of Quantitative Spectroscopy and Radiative Transfer, 1999. 62(2): p. 205-254.
158. Shirkey, R.C. Determination of atmospheric effects through EOSAEL. in 1988 Orlando Technical Symposium. 1988. International Society for Optics and Photonics.

159. Gordley, L.L., B.T. Marshall, and D. Allen Chu, LINEPAK: Algorithms for modeling spectral transmittance and radiance. Journal of Quantitative Spectroscopy and Radiative Transfer, 1994. 52(5): p. 563-580.

160. Majumdar, A.K. and J.C. Ricklin, Free-space laser communications: principles and advances. Vol. 2. 2010: Springer.

161. LaRocca, A.J. and R.E. Turner, Atmospheric transmittance and radiance: methods of calculation. 1975, DTIC Document.

162. Sabatini, R., F. Guercio, and S. Vignola, Airborne Laser Systems Performance Analysis and Mission Planning, in RTO-MP-046 Advanced Mission Management and Systems Integration Technologies for Improved Tactical Operations. 1999, NATO Research and Technology Organization (RTO): Florence, Italy.

163. Sabatini, R. and M.A. Richardson, RTO AGARDograph AG-300 Volume 26: Airborne Laser Systems Testing and Analysis. Systems Concepts and Integration Panel (SCI-126). 2010: NATO Science and Technology Organization.

164. Chu, T. and D. Hogg, Effects of precipitation on propagation at 0.63, 3.5, and 10.6 microns. Bell System Technical Journal, The, 1968. 47(5): p. 723-759.

165. Bisyarin V.P., B.I.P., Rubash V.K., Sokolov A.V., Attenuation of 10.6 and 0.63 Micron Laser Radiation. Radio Engineering and Electronic Physics 1971. 16: p. 1594-1597.

166. Fried, D.L., Statistics of a geometric representation of wavefront distortion. JOSA, 1965. 55(11): p. 1427-1431.

167. Brookner, E., Improved model for the structure constant variations with altitude. Applied optics, 1971. 10(8): p. 1960-1962.

168. Tatarski, V.I., R.A. Silverman, and N. Chako, Wave propagation in a turbulent medium. Physics Today, 2009. 14(12): p. 46-51.

169. Hufnagel, R. Variations of atmospheric turbulence. in Digest of Technical Papers, Topical Meeting on Optical Propagation through Turbulence. 1974.

170. Honkavaara, E., et al., Digital airborne photogrammetry-A new tool for quantitative remote sensing?-A state-of-the-art review on radiometric aspects of digital photogrammetric images. Remote Sensing, 2009. 1(3): p. 577-605.

171. Lei, F. and H.J. Tiziani, Atmospheric influence on image quality of airborne photographs. Optical Engineering, 1993. 32(9): p. 22712280.

172. Ochs, G., et al., Refractive-turbulence profiles measured by onedimensional spatial filtering of scintillations. Applied optics, 1976. 15(10): p. 2504-2510.

173. Chiba, T., Spot dancing of the laser beam propagated through the turbulent atmosphere. Applied optics, 1971. 10(11): p. 2456-2461.

174. Buck, A., Effects of the atmosphere on laser beam propagation. Applied optics, 1967. 6(4): p. 703-708.

175. Standard, U.M.L.S., in JSP 390. 2005, MoD Ordnance Board

176. U.S. Department of Transportation, FAA Order JO $7400.2 \mathrm{G}$ (Effective date: April 10, 2008). Subject: Procedures for Handling Airspace Matters. Part 6. Miscellaneous Procedures. Chapter 29. Outdoor Laser Operations.

177. FAA Advisory Circular 70-1 Subject: Outdoor Laser Operations (dated Dec. 30, 2004).

178. FAA 7400.2D Chapter 34, Outdoor Laser/High Intensity Light Demonstrations.

179. Deitz, P.H., Probability Analysis of Ocular Damage due to Laser Radiation Through the Atmosphere, Appl. Opt. 8, 371-375, (1969).

180. Hermann, J.A., Evaluation of the scintillation factor for laser hazard analysis, Appl. Opt., 29, 1287-1292, 1990.

181. Hill, R.J., Churnside, J.H. and Sliney, D.H. Measured statistics of laser beam scintillation in strong refractive turbulence relevant to eye safety, Health Phys. 53, 639-647, (1987).

182. Zilberman, A., Golbraikh, E. and Kopeika, N.S., Atmospheric scintillations and laser safety, 2011, pp. 816102-816102-12.

183. Sabatini, R. and Richardson, M.A. A new approach to eye-safety analysis for airborne laser systems light test and training operations, Optics \& Laser Technology 35, 191 - 198, (2003). 